\title{
DISPERSION OF RADIOACTIVE POLLUTANT IN A TORNADIC STORM
}

D. W. PEPPER

\section{TIS FILE * RECORD COPY}

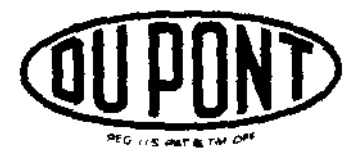

E. I. du Pont de Nemours \& Co. Savannah River Laboratory

Aiken, SC 29808 


\section{DISCLAIMER}

This report was prepared by E. I. du Pont de Nemours and Company (Du Pont) for the United States Department of Energy under Contract DE-ACO9-76SR00001 and is an account of work performed under that Contract. Neither the United States, the United States Department of Energy nor Du Pont, nor any of their employees, makes any warranty, express or implied, or assumes any iegal liability or responsibility for the accuracy, completeness, or usefulness of any information, apparatus, product, or process disciosed herein, or represents that its use will not infringe privately owned rights. Reference herein to any specific commerical product, process, or service by trade name, mark, manufacturer, or otherwise does not necessarily constitute or imply endorsement, recommendation, or favoring of same by Du Pont or by the United States Government or any agency thereof. The views and opinions of authors expressed herein do not necessarily state or reflect those of the United States Government or any agency thereof.

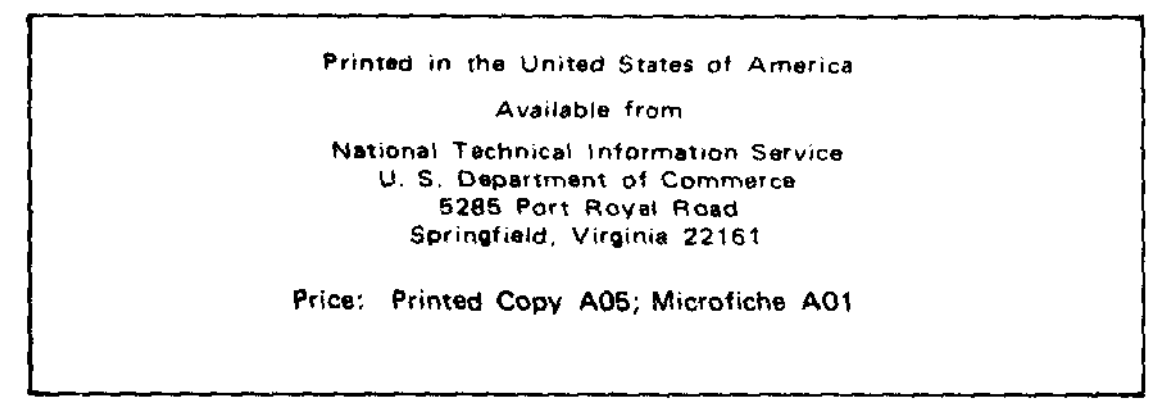


DP-1556

Distribution Category: UC-11

\section{DISPERSION OF RADIOACTIVE POLLUTANT IN A TORNADIC STORM}

Darrel1 W. Pepper

Approved by

Todd V. Crawford, Research Manager

Environmental Transport Division

Publication Date: May 1981

E. I. du Pont de Nemours \& Co. Savannah River Laboratory Aiken, SC 29808

PREPARED FOR THE U. S. DEPARTMENT OF ENERGY UNDER CONTRACT DE-ACO9.76SRO0001 
A three-dimensional numerical model is used to.calculate ground-level air concentration and deposition (due to precipitation scavenging) after a hypothetical tornado strike at a plutonium fabrication facility in Pennsylvania. Plutonium particles less than $10 \mu \mathrm{m}$ in diameter are assumed to be lifted into the tornadic storm cell by the vortex. The rotational characteristics of the tornadic storm are embedded within the larger mesoscale flow of the storm system. The design-basis translational wind values are based on probabilities associated with existing records of tornado strikes in the vicinity of the plant site. Turbulence exchange coefficients are based on empirical values deduced from experimental data in severe storms and from theoretical assumptions obtained from the literature. The quasi-Lagrangian method of moments is used to model the transport of concentration within a grid cell volume.

In all case studies, the effects of updrafts and downdrafts, coupled with scavenging of the particulates by precipitation, account for most of the material being deposited within $20-45 \mathrm{~km}$ downwind of the plant site. Ground-level isopleths in the $x-y$ plane show that most of the material is deposited behind and slightly to the left of the centerline trajectory of the storm. Approximately $5 \%$ of the material is dispersed into the stratosphere and anvil section of the storm. 
I. Introduction 7

II. Site Characteristics and Design Basis Tornado 7

III. Mathematical Model 9

IV. The Numerical Model 21

V. Results 30

VI. Model Sensitivity 39

A. Effect of Neglecting Diffusion 39

B. Effects of Wind Profiles 43

C. Effects of Updrafts and Downdrafts 43

D. In-cloud Scavenging (Rainout) 44

E. Height of the Storm 45

F. Effect of Topography (Surface Roughness Length) 45

VII. Conclusions 46

VIII. References 49

Appendix $\quad A-1$ 
1 Initial Dispersion Pattern Within the Tornadic Storm 11

2 Variation of Vertical Velocity within the Core of the Design Basis Tornado 13

3 Distribution of Updraft and Downdraft Velocities with Height in the Thunderstorm 15

4 Distribution of Vertical Winds at $1000 \mathrm{~m} \quad 16$

5 Mass Budget Within a Rotating Thunderstorm 20

6 Advection of a Single Cell of Concentration in a Two Dimensional Plane 24

7 Transport of a Cell of Concentration by Advection During One Time Step Using the Method of Moments 25

8 Air Concentration in the X-Z Plane (Center of Plane Passing Through Axis of Tornado) 32

9 Ground Level Air Concentration in the X-Y Plane ( $T=40 \mathrm{~min}$ ) (Rotational Winds Represent Tornadic Storm) 32

10 Maximum Ground-Leve1 Centerline Air Concentration from Initialization Point in Storm 33

11 Maximum Ground-Level Centerline Air Concentration from Plant Site 34

12 Ground-Level Air Concentration Isolog Plots $\left(\mathrm{m}^{-3}\right)$ in the $X-Y$ Plane $(T=60 \mathrm{~min}) \quad 35$

13 Ground-Leve1 Deposition Isolog Plots $\left(m^{-2}\right)$ in the X-Y Plane $(T=60 \mathrm{~min}) \quad 37$

14 Maximum-Ground Level Centerline Air Concentration $\left(\mathrm{m}^{-3}\right)$ Modified Gaussian Model 38

15 Concentric Annuli in $22.5^{\circ}$ Sectors in the X-Y Plane 40 


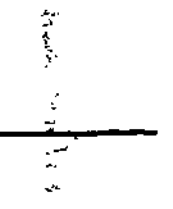

1 Windspeed Values $(\mathrm{m} / \mathrm{sec})$ and Probabilities of Occurrence 8

2 Diffusion Coefficient Parameters 18

3 Average Sector Air Concentration $\left(\mathrm{m}^{-3}\right)$ at Ground Level 41

4 Average Sector Deposition $\left(\mathrm{m}^{-2}\right)$ at Ground Level 42 
The risks and consequences of a tornado striking a nuclear facility and dispersing radioactive pollutants to the atmosphere are extremely difficult to determine. The mechanisms of formation and air motions associated with tornadoes and severe thunderstorms are not precisely known. The problem is compounded by the fact that the physics of dispersion in a severe storm is even less known. The scales of motion and degree of turbulence intensity vary dramatically from the tornado to the thunderstorm cell. The inability of a tornado-thunderstorm cell model to accurately account for this variability prevents exact predictions of radioactive dispersion. However, based on available knowledge, estimates and crude assumptions have been made to describe the general dispersion of a pollutant following a tornado strike. The hypotheses remain untested and the dispersion patterns unverified. 


\section{INTRODUCTION}

A series of studies, sponsored by the U.S. Nuclear Regulatory Commission and managed by Argonne National Laboratory, have been conducted regarding the radiological consequences of a hypothetical tornado striking a plutonium fabrication facility located within the contiguous U.S. A numerical model was developed to aid in the analysis of the meteorological dispersion of radioactive particulates following breachment of the facility. This report deals principally with the theoretical development of the tornado dispersion model.

Wind storm risk assessments and site characterizations for six plutonium fabrication facilities have been made by Fujital based on existing extreme wind and tornado records surrounding each individual plant site. The individual storm characteristics were computed as a function of yearly probabilities in an effort to determine a design-basis storm representative to each specific site.

The design-basis storm used in this study is based on the wind field model and site characteristics for one of the nuclear fabrication facilities near Leechburg, PA. The facility is assumed to be breached and a unit source of plutonium particles $(\sim 10 \mu \mathrm{m}$ in diameter) released into the tornado.

\section{SITE CHARACTERISTICS AND DESIGN BASIS TORNADO}

Site topography and characteristics along with wind speed values and probability of occurrence for each of the plutonium fuel fabrication sites are discussed in detail by Fujita. 1 Report number WASH- 1300 uses a probability of $10^{-7}$ per year in determining the frequency of design-basis tornadoes for nuclear power plants throughout the contiguous U.S. ${ }^{2}$ However, the wind speeds associated with the $10^{-7}$ probability in WASH-1300 are considered to be too high (Mehta, et al. ${ }^{3}$ and Fujita*).

This study uses a series of wind speeds associated with specific probabilities per year of a tornadic storm striking the nuclear facility. These values are based on the study conducted by Mehta, et al.3, who analyzed the structural responses of the

* T. T. Fujita, Personal Communication, 1977. 
buildings at each of the six sites in terms of threshold values of wind speed and consequences of damage (see Table 1). The radius of the tornado is assumed to be $150 \mathrm{~m}$ with the vortex extending to $1000 \mathrm{~m}$. Fujital reported that numerous tornadoes with wind speeds corresponding to the probabilities used in this report have actually occurred in the vicinity of the plant site. It is assumed that the design-basis tornado causes enough damage to the facility (Mishima, et al. ${ }^{4}$ ) to allow unencapsulated plutonium particles to be lifted into the vortex.

Fujital shows that most small- and medium-sized tornadoes have moved in an east-northeast direction near the site, while large-sized tornadoes (F4-F5) have moved in an east-southeast direction. Large-scale tornado strikes in the vicinity of each site are only based on a few years of data. In this study, a normalized direction for the hypothetical tornado is used to define the trajectory of the design-basis storm and pollutant cloud center. As more precise information on wind speeds and the directions of tornado-bearing storms becomes available, a more realistic estimate of the downwind dispersion patterns $c$ an be made. Normalization of the mean direction of transport enables the model to be used for other locations within the U.S.

A design-basis tornado model has been developed by Fujita 5,6 based on empirical evidence and analytical solutions to the governing equations of motion describing actual tornadoes. The model predicts flow fields and pressure drops surrounding the vortex and can account for how vertical velocity varies with height in the core of the tornado. The translational motion of the vortex can also be taken into account. Required input into the model has been kept to a minimum with maximum tangential wind speed and radius of the vortex key input parameters. The model also includes the presence of satellite (suction) vortices within the tornado. This tornado model should permit a more thorough analysis to be made with regards to residence time and uptake of debris within the vortex. In this study, the pollutant source is assumed to be totally lifted into the thunderstorm cell. The problem of dispersal about the vortex has been considered assuming on $1 y$ rather idealized concepts (Pepper ${ }^{7}$ ).

\section{TABLE 1}

\section{Windspeed Values $(\mathrm{m} / \mathrm{sec})$ and Probabilities of Occurrence}

$\begin{array}{llll}\text { Probability per year } & 10^{-7} & 10^{-6} & 7.2 \times 10^{-6} \\ \text { Maximum total } & 125 & 96.5 & 67.0 \\ \text { Translational } & 25 & 19.2 & 13.4 \\ \text { Tangential } & 100 & 77.3 & 53.6\end{array}$




\section{MATHEMATICAL YODEL}

The model developed for this study is based on the solution of the three-dimensional time-dependent equation for pollutant transport

$$
\frac{\partial X}{\partial t}+\vec{U} \cdot \nabla \chi=\nabla \cdot(\hat{K} \nabla \chi)+S
$$

where $x$ is the concentration $\left(\mathrm{g} / \mathrm{m}^{3}\right), \vec{U}$ is the vector velocity field $(\mathrm{m} / \mathrm{sec})$, $\hat{\mathrm{K}}$ is the directionally dependent eddy diffusivity (exchange coefficient of diffusion, $\mathrm{m}^{2} / \mathrm{sec}$ ), and $\mathrm{S}$ represents the sink terms associated with rainout, washout, and particle deposition $\left(\mathrm{g} / \mathrm{m}^{3}-\mathrm{sec}\right)$. The complexity of the flow fields associated with tornadic storms and the numerous scales of turbulence involved (which characterize the diffusion processes) do not permit simple solutions to Equation 1 .

Analytical methods, used in the past to solve Equation 1 under ideal steady-state conditions, are not flexible enough to include variations in updrafts and downdrafts or vertical shear within the thunderstorm cell. A study by Pepper ${ }^{7}$ assumed that a pollutant cloud (puff) reached a specific height in the thunderstorm cell, and then diffused in a Gaussian manner as if originating from that height. The effects of wind shear were neglected. Eddy diffusivities were based on energy dissipation rates measured in thunderstorms. The downwind diffusion following decay of the tornadic storm was calculated from extrapolations of the similarity theory.

It is unlikely that such extrapolations are entirely accurate for severe thunderstorms. If a model has to accommodate the temporal and spatial variations of numerous meteorological parameters, including the effects of wind shear and wet deposition, a more realistic approach must be made. The effects of wind shear on the pollutant cloud and in-cloud scavenging can contribute dramatically to the early deposition of radioactive particles downwind of the plant site (Davis 8 , Hane ${ }^{9}$ ).

A set of boundary conditions must be specified so the solution will be constrained to the domain of interest. In order to facilitate the solution of Equation 1, the following set of boundary conditions is used

$$
\left.\begin{array}{rl}
\mathrm{K}_{z z} \frac{\partial X}{\partial z}=0 & z=0, \mathrm{H} \\
\frac{\partial^{2} X}{\partial \mathrm{x}^{2}}=0 & \mathrm{x}=0, \mathrm{x}_{\max } \\
\frac{\partial^{2} X}{\partial \mathrm{y}^{2}}=0 & \mathrm{y}=0, \mathrm{y}_{\max }
\end{array}\right\}
$$


where $H=$ height of the thunderstorm cell and acts as a partial lid to the concentration. The lack of well-posed boundary conditions does not cause serious problems since the advection terms tend to dominate the diffusion terms in Equation 1.

The initial dispersion conditions are crucial to the downwind dispersal patterns following breachment of the facility. In order to simplify the source term requirements of the model, a unit source of radioactive pollutant per $\mathrm{m}^{3}$ (uniformly distributed) is used. This unit source of radioactive pollutant is assumed to be picked up by the tornado and lifted into the thunderstorm cell. The puff consists of particles which have diameters less than $10 \mu$. Once the pollutant reaches the thunderstorm cell (at a point where the vertical velocity of the tornado is less than the updraft velocity of the thunderstorm cell), the puff is assumed to be dispersed by dynamics of the thunderstorm cell (Jessup 10 ). The puff becomes dispersed throughout the thunderstorm cell within about 20 minutes according to the mass balance assumptions suggested by Fujita*. Figure 1 shows the initial source distribution after 20 minutes within the thunderstorm.

The concentration pattern is limited to the size of the thunderstorm cloud with a maximum peak at each level near the position of the tornado axis. Since the values of advection velocities and atmospheric turbulence are high, it seems reasonable to assume the pollutant will become well mixed throughout the thunderstorm cell (Pasquil1**). The initial concentration within the thunderstorm cell is obtained by using a skewed log-normal distribution with maximum values centered on the axis of the tornado. Once the initial distributions are established, advection, turbulent diffusion, and rainout scavenging act on the pollutant cloud.

The three-dimensional wind vector, $U$, is required at each time-step of integration. Unfortunately, the mesoscale wind field associated with the tornadic storm is a difficult problem to accurately model. Correct calculation of the trajectory of the storm and pollutant dispersion pattern is directly dependent upon the wind field structure (Davies-Jones 11 ). Subsequently, knowledge of the thunderstorm cell dynamics is also essential to the transport of the polluant. Considerable effort has been spent in recent years to analyze the surrounding wind field structure (Fankhauser, 12 and Burgess, et a1.13). The use of Doppler radar has enabled researchers to actually measure the three-dimensional wind components in severe and tornadic storms, including the surrounding mesoscale flow field (Eagleman and Lin 14, Kropf $1 i^{1}$ and Miller 15 , Brandes 16 , Heymsfield 17 , Barnes ${ }^{18}$, Ray ${ }^{19}$, Miller $^{15}$, Hoxit, et a1. ${ }^{20}$,

\footnotetext{
* T. T. Fujita, Personal Communication, 1977.

** F. Pasqui11, Personnal Communication, 1976.
} 
Skewed Log-Normal Cell Values

\begin{tabular}{ccccccc}
\hline 0 & 0 & 0 & 0 & 0 & 0 & 0 \\
0 & .230 & .130 & .050 & .022 & .009 & .004 \\
0 & .130 & .090 & .040 & .020 & .009 & .004 \\
0 & .050 & .040 & .030 & .010 & .006 & .003 \\
0 & .022 & .020 & .010 & .008 & .004 & .002 \\
0 & .009 & .009 & .006 & .004 & .003 & 0 \\
0 & .004 & .004 & .003 & .002 & 0 & 0
\end{tabular}

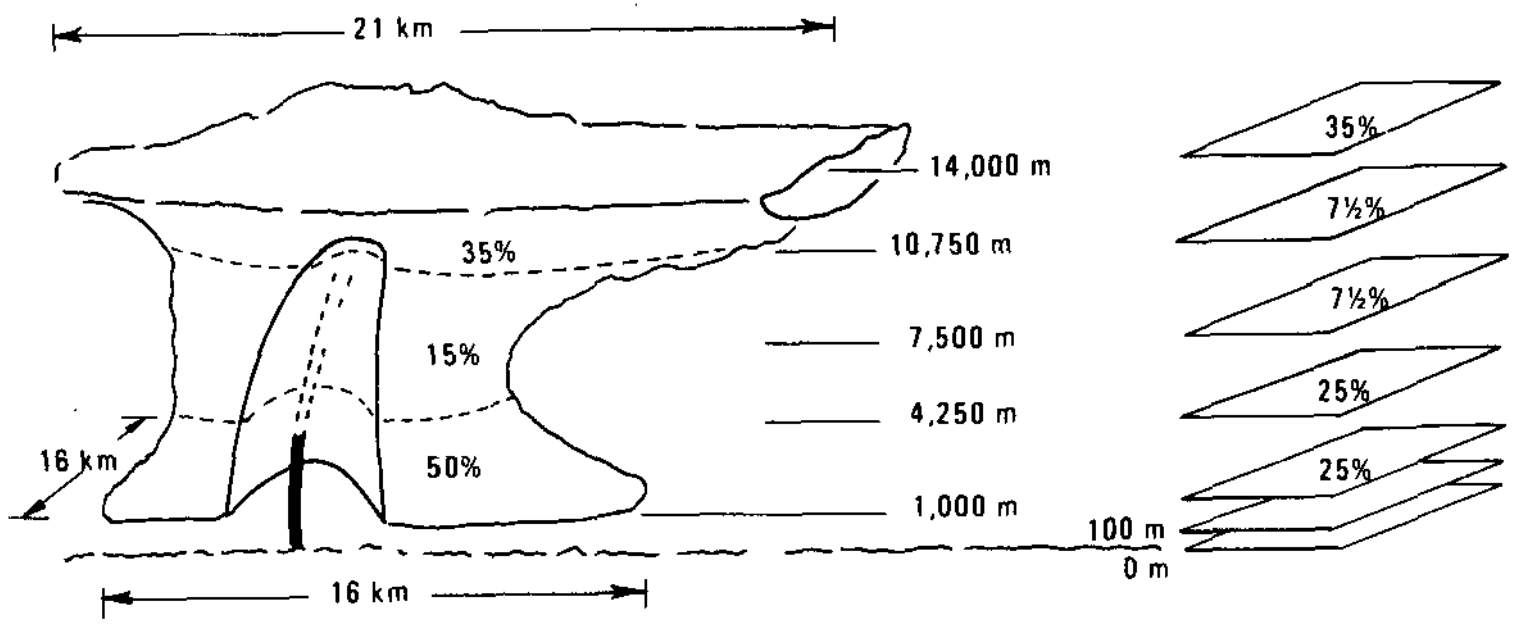

Figure 1. Initial Dispersion Pattern Within the Tornadic Storm 
Ray, et al.21). Similarly, work on modeling the three dimensional structure of severe storms has also been undertaken with some success (Klemp and Wihelmson 22 , Thorpe and Miller 23, Lin and Chang 24 Schlesinger 25 ). In this study, a three-dimensional solution of the equations of motion governing the thunderstorm cell dynamics was not undertaken. In further studies regarding dispersion within severe storms, emphasis should be placed on establishing more accurate storm cell and mesoscale wind fields surrounding the vortex.

The three-dimensional wind structure of the tornado (vortex) has been analyzed extensively by numerous investigators (see Golden and Purce $11^{26}$, Fujita6, Davies-Jones and Kessler 27 , and Rotunno 28 for a more complete review of the laboratory, field measurements, and numerical simulation experiments). Unfortunately the scales of motion involved in the vortex are much less than those with in the thunderstorm cell. It is the large scale flow field of the thunderstorm which ultimately determines the dispersion pattern of the pollutant, not the uptake and radial inflow of the shorter lived tornado.

The variation of the vertical velocity within the core of the tornado (Fujita ${ }^{6}$ ) is shown in Figure 2 . This velocity variation was used as a guide to determine the amount of time required for the vortex to lift the pollutant source into the thunderstorm cell.

In order to create a plausible mesoscale three-dimensional wind field of the design-basis tornadic storm striking the nuclear facility, a set of empirical relations are used based on the work of Fujita ${ }^{6}$, Orville 29 , Newt on 30 , and Eagleman and Lin 13 . A subjective analysis and interpolation scheme was used to generate a mass-consistent three-dimensional wind field (Pepper and Baker ${ }^{31}$ ). The horizontal winds outside the storm cell are assumed to be a function of height $(z)$ and distance ( $x$ ) such that

$$
\begin{aligned}
& \left.u(z, t)=2.2 z^{\cdot 18(m / s e c}\right) ; \quad z<1000(m) \\
& u(z, t)=\left\{\begin{array}{l}
25.0(\mathrm{~m} / \mathrm{sec}) \\
19.2(\mathrm{~m} / \mathrm{sec}) \\
13.4(\mathrm{~m} / \mathrm{sec})
\end{array} \quad 1000 \leqslant \mathrm{z}<\mathrm{H}\right. \\
& \mathrm{u}(\mathrm{z}, t)=1.25\left\{\begin{array}{l}
25.0(\mathrm{~m} / \mathrm{sec}) \\
19.2(\mathrm{~m} / \mathrm{sec}) \\
13.4(\mathrm{~m} / \mathrm{sec})
\end{array} \quad \mathrm{z} \geqslant \mathrm{H}\right.
\end{aligned}
$$

Accurate knowledge of the lateral wind field values would more correctly introduce the meandering and lateral shifting of the storm's actual direction. However, information regarding this velocity component is limited (with regards to describing the general nature of tornadic storms). In this study, the translational velocities of the three tornadic storm cells are assumed to be constant.' 


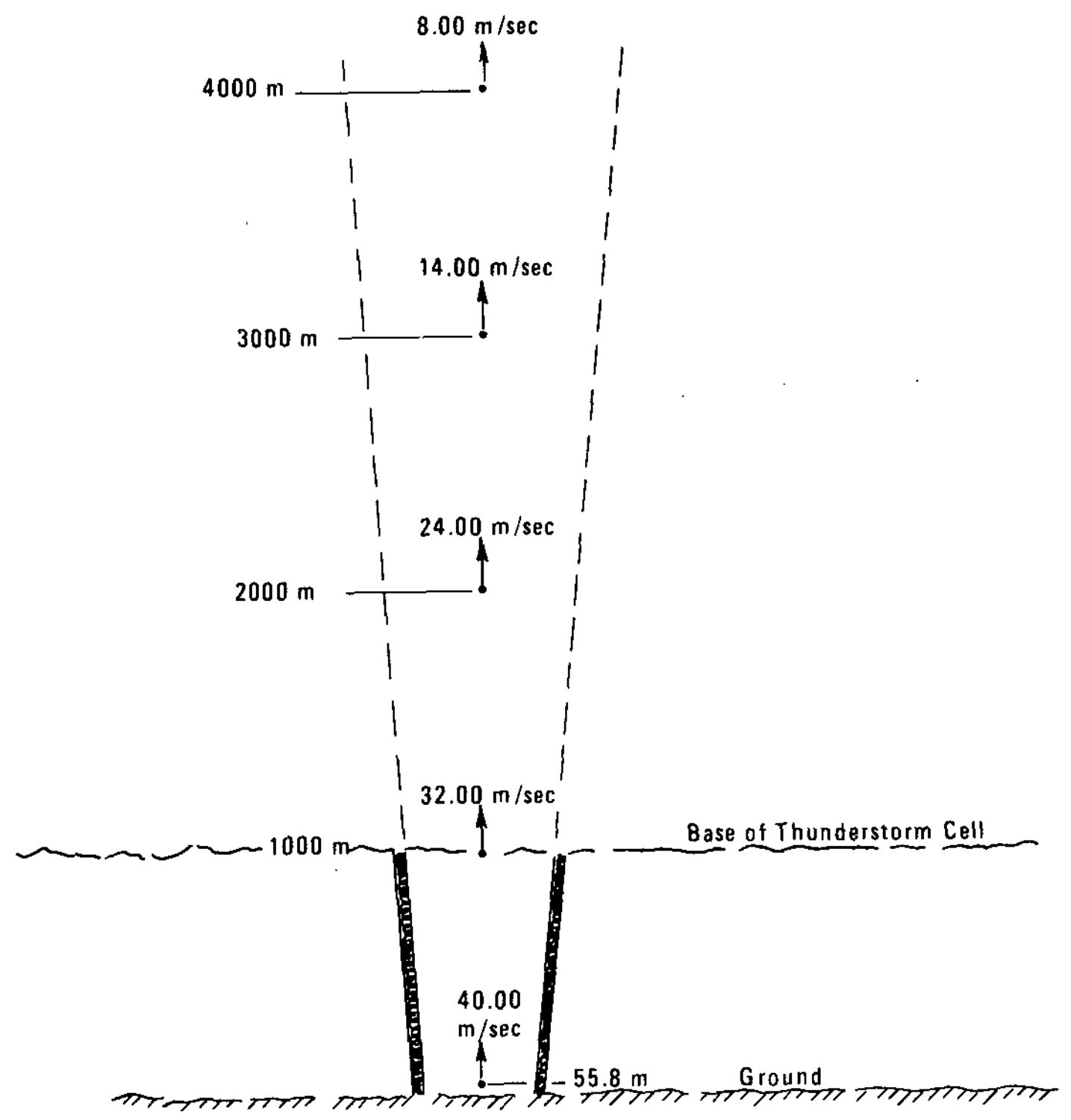

Figure 2. Variation of Vertical Velocity Within the Core of the Design Basis tornado 
The distribution of vertical winds in the tornadic storm cell is shown in Figure 3. The updraft and downdraft velocities are both allowed to increase logarithmically to specific heights within the thunderstorm cell and then decrease to zero at the top of the anvil, $H$. The magnitudes of the vertical wind speeds were chosen to be appropriate for the intensity of the tornadic storms and are comparable to observations and measurements of vertical velocities within severe storms (Browning and Donaldson, 32 Fujita ${ }^{6}$ ). The horizontal distribution of the vertical velocity field is shown in Figure 4 for the $1000 \mathrm{~m}$ level (corresponding to the base of the thunderstorm cell). The vertical distribution of the updraft and downdraft velocities is multiplied by the appropriate horizontal contour values to obtain the vertical component of the wind throughout the cell.

Advection and diffusion of the horizontal distribution of the vertical velocity field (at the $1000 \mathrm{~m}$ level of the cloud) enable the updraft and downdraft regions of the storm to be propagated with the trajectory of the storm. The rotational characteristics of the horizontal wind field within the storm cell are similarly progagated with the trajectory of the storm (analogous to the vertical velocity field calculation). The role of the vertical wind distribution tends to keep the pollutant well mixed initially throughout the storm cell. The rotational characteristics of the storm cell, on the other hand, continually shift the pollutant into the rearward and downdraft regions of the storm. This coupling of the two wind fields tends to create a somewhat unrealistic decoupling of the pollutant distribution, i.e., the downdraft velocities act more to bring the pollutant to the ground than keep it well mixed within the storm. Subsequent work on a more accurate velocity distribution within the storm cell may alleviate this characteristic of the present model. As a result of this decoupling effect, pollutant amount and time in reaching the surface is considered to be conservative.

To calculate the eddy diffusivities of air pollutants under the simplest of conditions is a classical problem. Little is known about their behavior except near the earth's surface (Shir and Shieh ${ }^{33}$ ). A number of empirical relations have been obtained for varying atmospheric stabilities. However, essentially no information is available for eddy diffusivities associated with the highly unstable nature of tornadoes and thunderstorm cells. While some experiments have been conducted on the distribution of chaff in thunderstorms, the data is sparse and the chaff are much larger in size than the plutonium particles considered in this study. Hence the dispersion patterns in chaff could be markedly different than that of plutonium particulates. Since precise formulation of the diffusion coefficients is intractable, a set of empirical parameters is used. Based on the work of Ragland and 


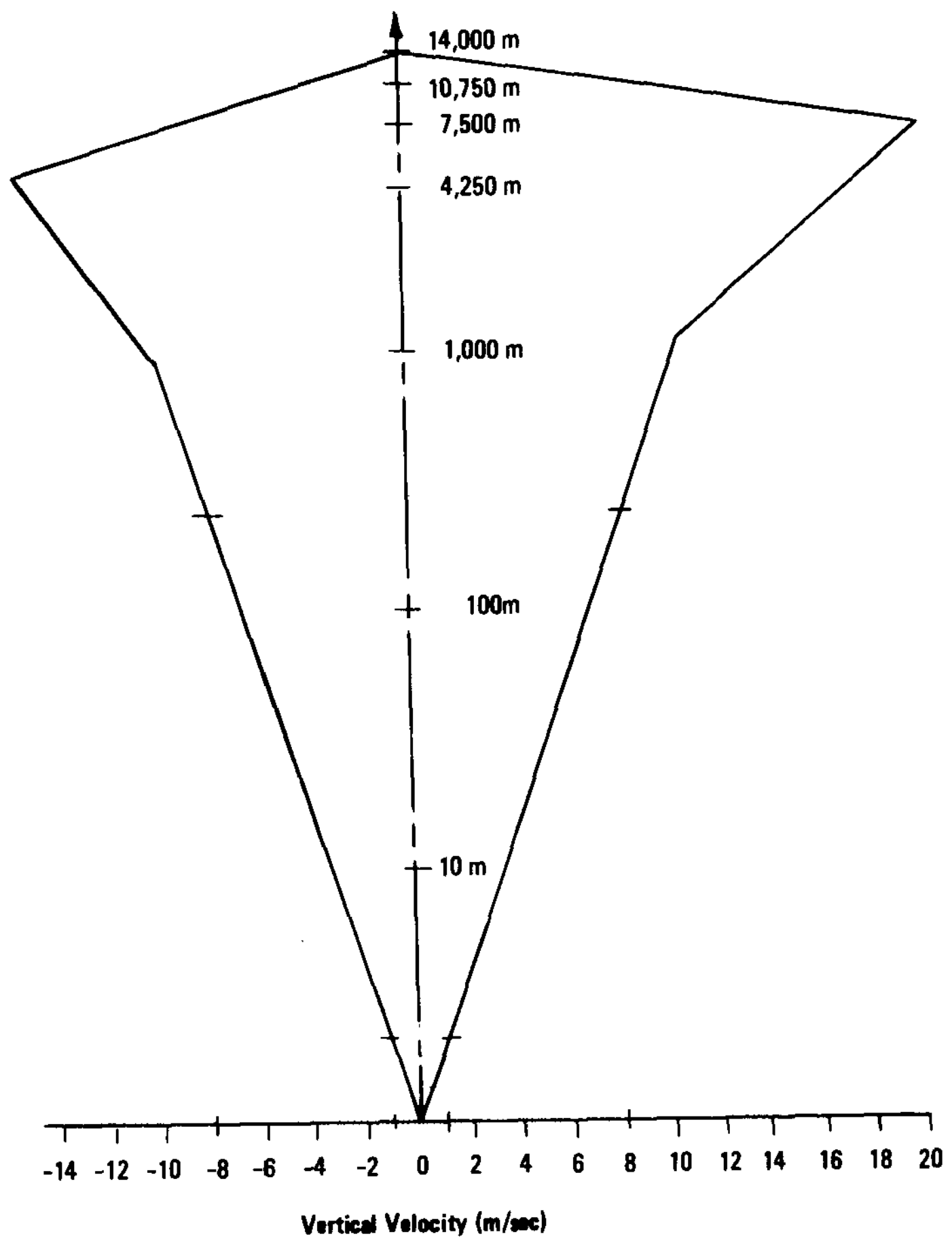

Figure 3. Distribution of Updraft and Downdraft Velocities with Height in the Thunderstorm 


\begin{tabular}{|c|c|c|c|c|c|c|}
\hline \multicolumn{7}{|c|}{ Truied Coll Volme $(\mathrm{m} / \mathrm{mes})$} \\
\hline-1.0 & -3.5 & -3.5 & -1.0 & 0. & 0. & 0 \\
\hline-3.5 & -10.0 & -3.5 & -1.0 & 0. & 0. & \\
\hline-3.5 & -1.0 & 0. & 2.0 & 0. & 0. & \\
\hline-1.0 & 2.0 & 3.5 & 10.0 & 2.0 & 0. & \\
\hline 0. & 1.0 & 3.0 & 3.5 & 3.5 & 2.0 & \\
\hline 0. & 0. & 0. & 0. & 0. & 0. & \\
\hline 0. & $\mathbf{0 .}$ & 0. & 0. & 0. & 0. & \\
\hline
\end{tabular}

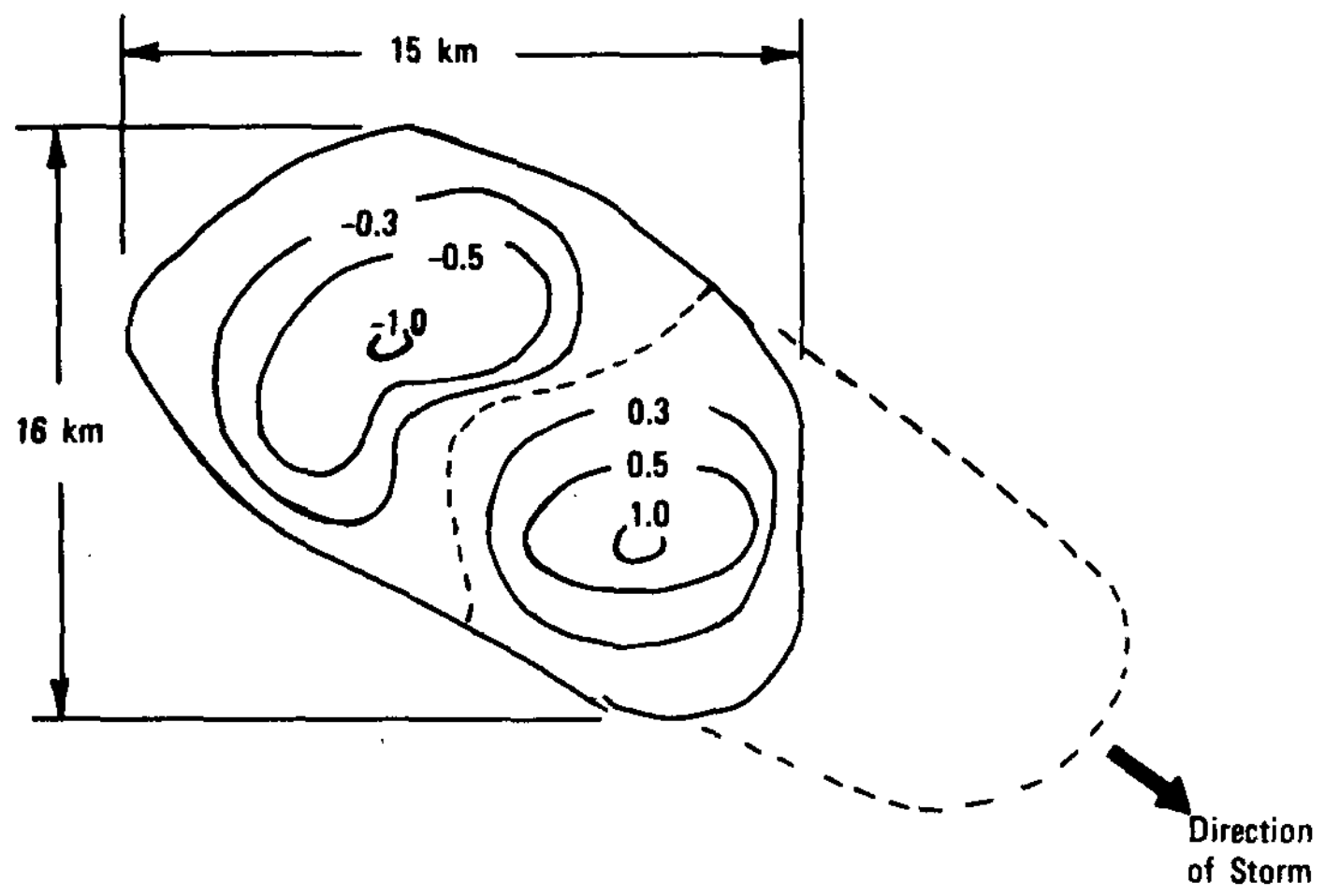

Figure 4. Distribution of Vertical Winds at $1000 \mathrm{~m}$ 
Dennis ${ }^{34}$, Pasquil110, Crawford*, Pepper 7 , Agee 35 , Frisch 36 , and Hildebrand 37 , the following general equation is used to describe the turbulent diffusion coefficients within a thunderstorm cells

$$
\hat{\mathrm{K}}=\frac{1}{2}\left(\frac{\mathrm{d} \sigma^{2}}{\mathrm{dt}}\right)=\sigma \frac{\mathrm{d} \sigma}{\mathrm{dt}}
$$

or

$$
\hat{\mathrm{K}} \cong \frac{\sigma^{2}}{\mathrm{nt}}=\mathrm{G} \sigma^{2-\mathrm{n} / \mathrm{n}}
$$

where $\sigma^{2}=G t$ and $G$ has dimension of $\mathrm{m}^{2} / \mathrm{sec}$. In order to account for vertical variation in turbulence intensity, Equation 4 is premultiplied by $\mathrm{Az}^{\mathrm{K}}$ such that

$$
\hat{\mathrm{K}}=A z^{\mathrm{K}} \sigma^{2-\mathrm{n} / \mathrm{n}}
$$

Equation 5 is assumed to be valid for $t<20$ minutes. Individual values for $A, K, C, \sigma$, and $n$ are given in Table 2 to be easily changed. When $t>20$ minutes, a different set of relations is used to account for the decrease of turbulent intensity following decay of the thunderstorm to very unstable atmospheric conditions. The expressions are:

$$
\begin{aligned}
& K_{x}=D(\bar{u} t)^{\alpha} /(2 t) \\
& K_{y}=E(\bar{u} t)^{\alpha} /(2 t) \\
& K_{z}=F u *_{z_{0}}\left(z / z_{0}\right)^{\beta}
\end{aligned}
$$

where $u$ is the translational velocity of the storm, $t$ is time, $D=0.004\left(1-.075 \log _{10} z\right), E=0.0075\left(1-.075 \log _{10} z\right), \sigma=7 / 4$, $F=0.41, \beta=.45, z_{0}$ is the surface roughness, $u^{*}$ is the friction velocity defined by $u^{*}=1.2 \mathrm{C}_{\mathrm{g}} \mathrm{U}_{\mathrm{g}}\left(\log _{10} \mathrm{R}_{\mathrm{O}}-1.8\right)$, with

* T. V. Crawford, Personnal Communication, 1977. 
$\mathrm{C}_{\mathrm{g}}$ being the geostrophic drag coefficient, $\mathrm{U}_{\mathrm{g}}$ the eostrophic wind, and $R_{o}=U_{g} / z_{O} f$ where $f$ is the coriolis parameter. The empirical constants appearing in Equation 6 were obtained from Ragland and Dennis ${ }^{34}$ and Lettau 38 for unstable atmospheric conditions. The effect of topography is not included in the advective wind field analysis,* but is introduced through the diffusion coefficients by the surface roughness parameter, $\mathrm{z}_{\mathrm{O}}$.

F1ux boundary conditions are important at $z=0$ and $z=H$. Neglecting resuspension, the surface is assumed to act as an absorber of plutonium particles (whether in the form of rain or dry particulates). When the flux of vertical concentration is set to zero at the surface, the surface acts as a perfect reflector. In order to account for deposition velocities at the surface, the flux at the ground is expressed in terms of a deposition velocity, $p\left(\right.$ Calder $\left.{ }^{39}\right)$, such that

$$
\lim _{z \rightarrow 0}[-F(z)]=p \lim _{z \rightarrow 0}[C(z)]
$$

and

$$
\lim _{z \rightarrow 0}\left[K_{z} \frac{\partial C}{\partial z}+\left(V_{g}-p\right) C(z)\right]=0
$$

\section{TABLE 2}

\begin{tabular}{|c|c|c|c|c|}
\hline$\underline{K}$ & A & $\underline{K}$ & C & $\sigma$ \\
\hline $\mathrm{K}_{\mathrm{x}}$ & 0.10 & 0.20 & 0.16 & $t$ \\
\hline$y$ & 0.10 & 0.20 & 3 & $t$ \\
\hline . & 0.60 & 0.20 & $\frac{3600-t}{t}$ & 1 \\
\hline
\end{tabular}

\section{Diffusion Coefficient Parameters}

*Topographic effects on pollutant dispersion are discussed in Pepper and Baker 35 and Reynolds, et al.40 
where $v_{g}$ is the actual settling velocity. By expressing the flux at the surface in form

$$
F=-(1-r) K_{z} \frac{\partial C}{\partial z}
$$

where $0<r<1$ is the reflection coefficient, Equations 7 through $9 \mathrm{can}$ be combined to give

$$
p C \approx v_{g} C+\left(K_{z} \frac{\partial C}{\partial z}\right)_{z=0} \approx(1-r) k_{z}\left(\frac{\partial C}{\partial z}\right)
$$

Thus, varying the value of $r$ from 0 to 1 will simulate the effect of losses at the surface by deposition (Kao4l).

The flux at the top of the cloud is set to transport 5 percent of the concentration within that layer to the stratosphere according to the mass balance of a rotating thunderstorm as suggested by Fujital. Figure 5 shows the mass balance of a tornadic storm with 5 percent of the concentration eventually reaching the stratosphere. The remaining 95 percent of the concentration is assumed to be well mixed within the thunderstorm cell. The top of the anvil is assumed to act as a vertical lid. The 5 percent injected into the stratosphere could be advected and diffused globally as fallout with continual entrainment between the tropopause and the stratosphere (Crawfor $\mathrm{d}^{*}$ ). Because these dispersion conditions usually have long-term effects spread over very large regions, the 5 percent mass lost out the top of the anvil is considered to have negligible immediate effect on the population.

Scavenging of the plutonium particles may occur by rainout. Based on the work of slinn 42 , the sink term in Equation 1 is written as

$$
S=-\psi C
$$

where the removal rate, $\psi$, is obtained from the relation (for in-cloud and below-cloud scavenging)

$$
\psi=\int_{0}^{\infty} d R N(r) \pi R^{2} E(a, R) V_{t}(R)
$$

* T. V. Crawford, Personal Communication, 1977. 
ST RATOSPHERE

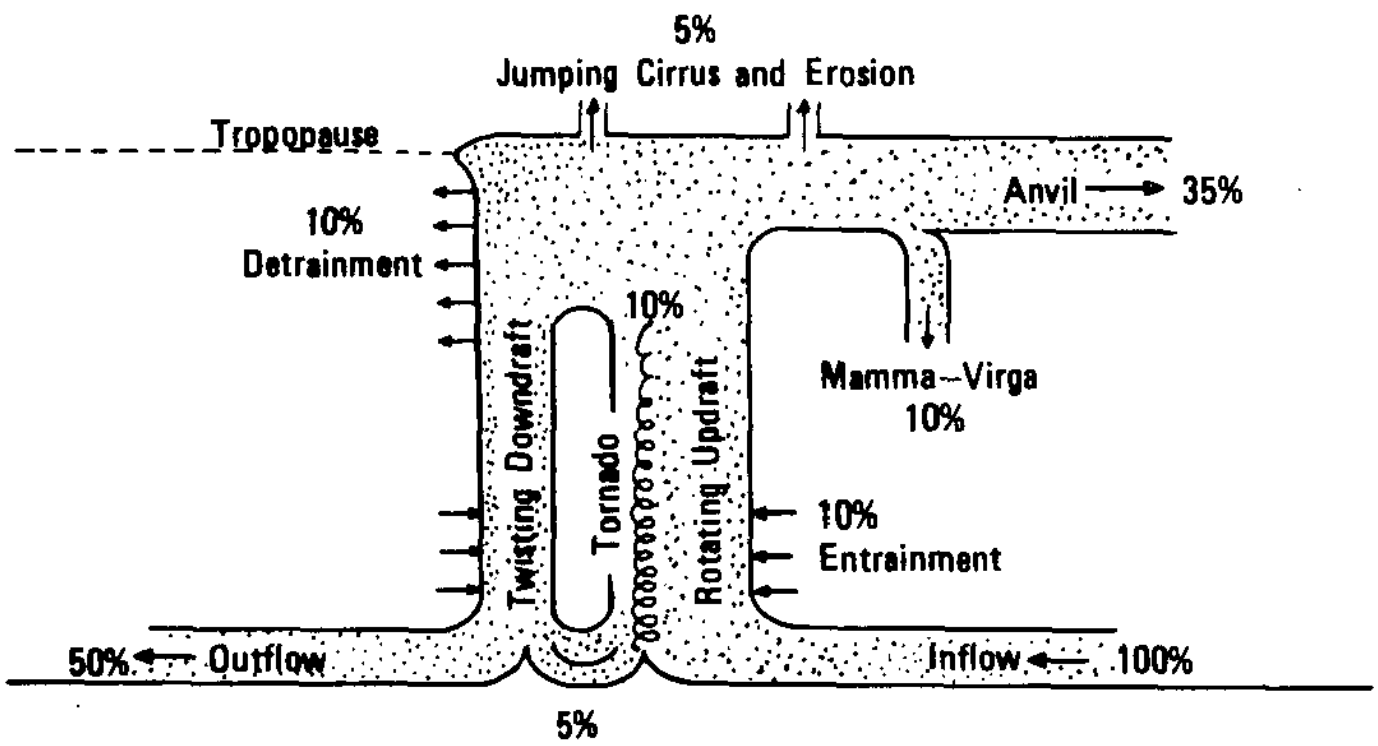

Recycling

Figure 5. Mass Budget Within a Rotating Thunderstorm 
with $N(r)$ being the number distribution function for liquid hydrometers in a cloud, $E(a, R)$ is the collision efficiency, $V_{t}(R)$ is the drop's terminal velocity, $a$ is the particle radius and $R$ is the droplet radius. In order to simplify the expression for the removal rate, Equation 12 is approximated by the empirical expression

$$
\psi \simeq C_{1} \frac{J_{0}}{R_{m}} E\left(a, R_{m}\right)
$$

Where $\mathrm{C}_{1}$ is an empirical constant which varies from 0.5 to $1.0, \mathrm{~J}_{0}$ is the rainfall rate, $R_{m}$ is the mean droplet size, $E$ is the collision efficiency ( $\equiv 1)$, and $a$ is the radius of the particulate. The relation for $\Psi$ is obtained in part from work by Davis ${ }^{9}, \operatorname{Slinn}^{4}$, Dingle 44 , and Hane 9 . In order to maintain mass conservation6, in the model, the amount of material scavenged in Equation 11 is added to the surface concentration on the ground $\left(\mathrm{g} / \mathrm{m}^{2}\right)$.

\section{THE NUMERICAL MODEL}

The model developed in this study is based on the solution of the three-dimensional time-dependent equation for pollutant transport as given by Fquation 1. Since little is known about the physics of motion in tornadic storms, a great deal of empiricism must be used. In order to accormodate the numerous velocity and diffusion values which exist during the lifetime of a tornado, a numerical model has been developed.

Release of radioactive material into a tornadic storm begins as a small scale dispersion problem which quickly develops into a mesoscale problem. Mesoscale problems normally require solution of three-dimensional equations. In order to keep computer running times and storage to a minimum, mesh spacing intervals are usually increased. However, if the mesh spacing is larger than the dimensions of the pollutant cloud, the mesh spacing is too coarse to accurately resolve steep gradients. Hence, the mesh spacing must either be redefined or subgrid scale monitoring of the concentration be maintained. Although Lagrangian marker particles are very useful in visualizing concentration transport and diffusion, their use is time consuming since the particle sum must be calculated for each cell. A particle-in-cell approach was attempted based on the methods used by Sklarew 45 and Lange 46 , but was found to be uneconomical and required excessive core storage.

In order to overcome the problems of numerical dispersion errors and mesh refinement inherent in most numerical schemes, a quasi-Lagrangian technique is coupled with a method which maintains subgrid scale resolution. To further reduce the core requirements needed to solve the three-dimensional equation of 
concentration transport (and winds), Equation 1 is split into a. series of one-dimensional advection-diffusion equations such that

$$
\begin{gathered}
\frac{\partial C^{*}}{\partial t}+u \frac{\partial C}{\partial x}-\frac{\partial}{\partial x}\left(K_{x} \frac{\partial C}{\partial x}\right)=s_{x} \\
\frac{\partial C^{* *}}{\partial t}+v \frac{\partial C^{*}}{\partial y}-\frac{\partial}{\partial y}\left(K_{y} \frac{\partial C^{*}}{\partial y}\right)=s_{y} \\
\frac{\partial C^{n+1}}{\partial t}+w \frac{\partial C^{* *}}{\partial z}-\frac{\partial}{\partial z}\left(K_{z} \frac{\partial C^{* *}}{\partial z}\right)=S_{z}
\end{gathered}
$$

This method involves splitting each individual one-dimensional equation into a Lagrangian advection part plus an Eulerian diffusion part. The method of second moments is used to maintain subgrid scale resolution of the concentration.

The method of second moments is a unique quasi-Lagrangian scheme initially developed by Egan and Mahoney 47 to accurately model the transport of urban pollutants. The method calculates the zeroth, first, and second moments of the concentration within $a$ mesh and then advects and diffuses the concentration by conserving the moments. The moments correspond to the mean concentration, center of mass, and scaled distribution variance (moment of inertia), respectively, and are given by

$$
\begin{gathered}
C_{m}=\int_{0.5}^{0.5} \mathrm{C}\left(\xi_{m}\right) d \xi \\
F_{m}=\int_{0.5}^{0.5} \mathrm{C}\left(\xi_{m}\right) \xi_{m} \mathrm{~d} \xi / C_{m} \\
R_{m}^{2}=12 \int_{0.5}^{0.5} \mathrm{C}\left(\xi_{\mathrm{m}}\right)\left(\xi_{\mathrm{m}}-\mathrm{F}_{\mathrm{m}}\right)^{2} \mathrm{~d} \xi / \mathrm{C}_{\mathrm{m}}
\end{gathered}
$$


where subscript $m$ denotes cell location in one-dimensional space, and $\S_{m}$ denotes the relative displacement of material within the $\mathrm{m}^{\text {th }}$ cell from the center of the cell. $\delta_{\mathrm{m}}$ varies from -0.5 to 0.5 corresponding to the left and right hand extreme boundaries of a cell. The length of the cell is non-dimensionalized by the grid element length (width or height, depending on the direction of the calculation). For simple rectangular concentration distributions (rectangular mesh geometry), the integrals are evaluated by summing the concentration distributions remaining and newly transported into each grid element for each time step. The advection of a single cell of concentration in two dimensions with the Courant numbers $(u \Delta T / \Delta s)$ equal to 0.5 is shown in Figure 6 . Simple advection tests in one dimension showed that a single cell of concentration could be advected accurately with time without numerical dispersion or computational damping errors. Further test cases with two- and three-dimensional advection of both single and multiple cells of concentration showed no numerical dispersion errors or minimal damping (Pepper and Long ${ }^{48}$ ). Similar tests with both finite difference and finite element techniques by Long and Pepper 49 showed either 1) severe spreading of the concentration due to computational damping, or 2) generation of wave packets of plus and minus concentration due to numerical dispersion. Since the dispersion of concentration within a tornadic storm is essentially one of advection, the second-moment technique appears to be applicable to the three-dimensional equation of concentration transport. The diffusion of concentration is of secondary importance during the early stages of dispersion within the storm cell; hence a simple, explicit centered difference scheme is used to approximate the second-order gradient transfer terms.

The downwind transfer of concentration by advection depends upon the value of the portioning parameter, $P_{m}$, where $P_{m}$ is defined (Egan and Mahoney ${ }^{47}$ )

$$
P_{m}=\left(F_{m}+\lambda+\frac{R_{m}}{2}-0.5\right) / R_{m}
$$

and $\lambda$ is the Courant number. If $P_{m}<0$, the concentration is not advected into the $\mathrm{m}+1$ cell. If $\mathrm{P}_{\mathrm{m}}>0$, all of the concentration is advected into the downwind cell. For $0<\mathrm{P}_{\mathfrak{m}}<1$, a fraction of the concentration $P_{m} C_{m}$ is advected while $\left(1-P_{m}\right) C_{m}$ remains behind. Figure 7 shows the scaling parameters involved in the advection of a rectangular concentration distribution in one dimension during one time step. The center of mass of the distribution is given at $-0.5+P_{m} R_{m} / 2$ relative to the center of the $m+1$ cell and has a horizontal spread equal to $P_{m} R_{m}$ in the new cell. The amount left in cell $\mathrm{m}$ has a center of mass at $\left(1-R_{m}+P_{m} R_{m}\right) / 2$ with a horizontal spread of $\left(1-P_{m}\right) R_{m}$. 

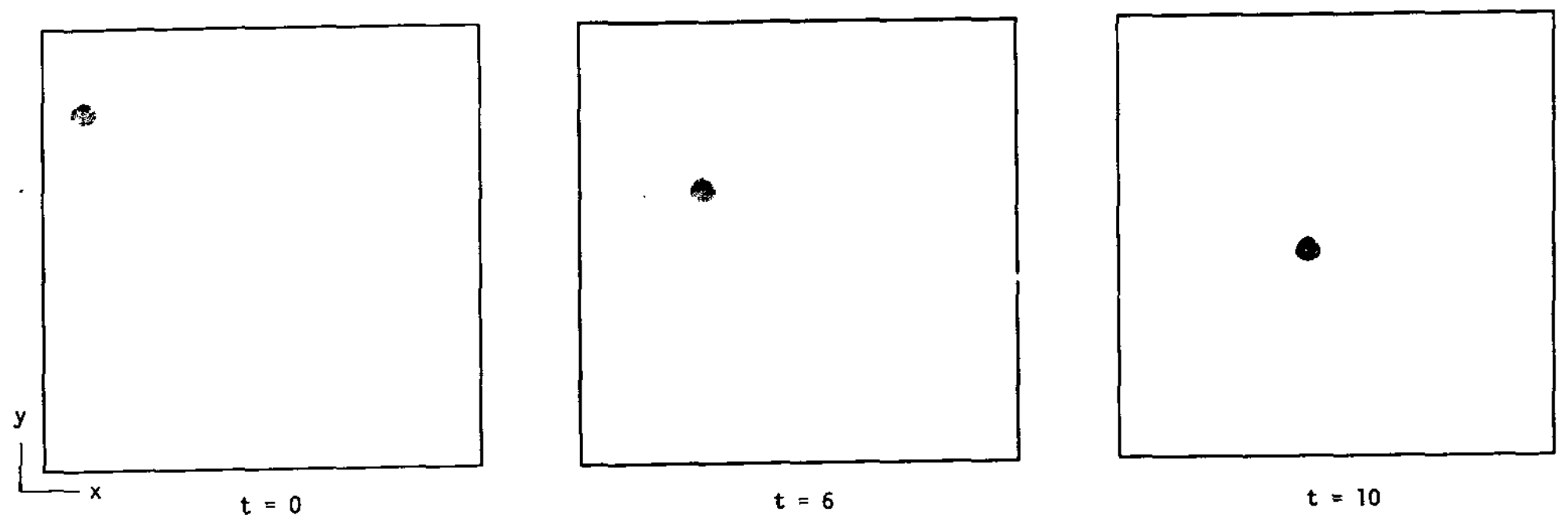

ก
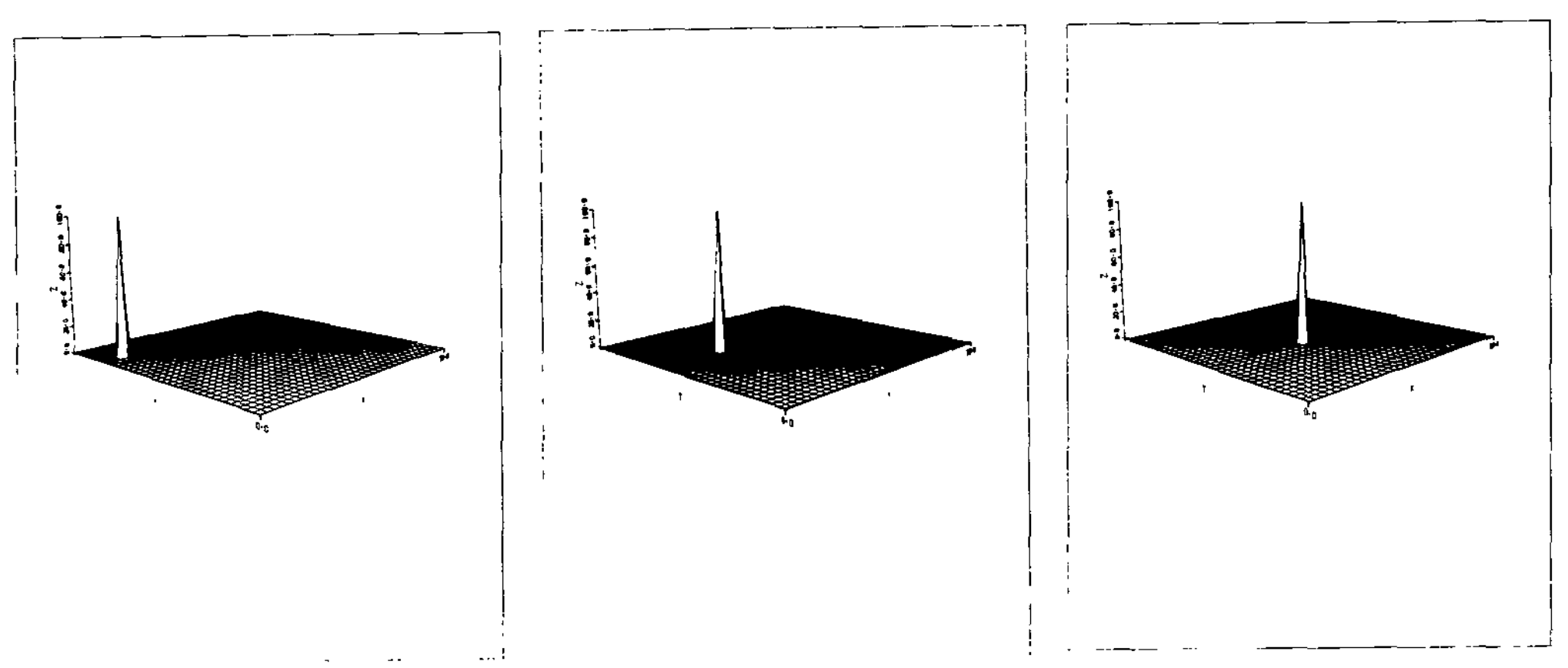

Figure 6. Advection of a Single Cell of Concentration in a Two Dimensional Plane 

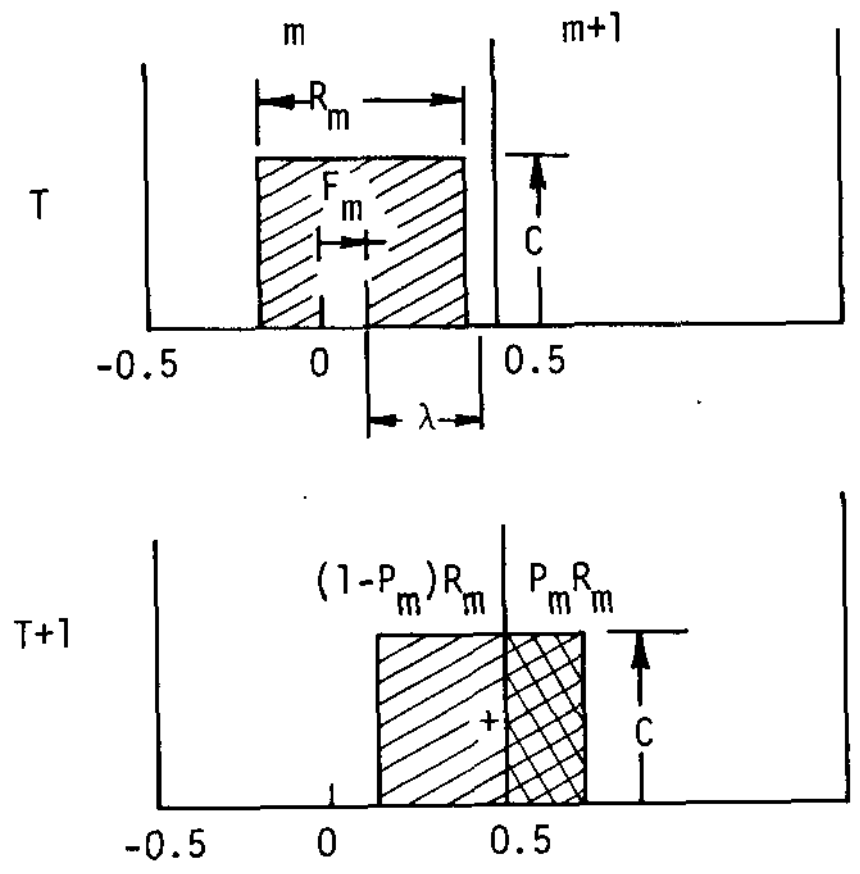

Figure 7. Transport of a Cell of Concentration by Advection During One Time Step Using the Method of Moments 
Extension of the method of moments to two and three dimensions is straightforward as based on the rules for the one-dimensional example just discussed (Pepper and Baker ${ }^{31}$ ). Since the zeroth through second moments are generally evaluated from concentration distributions advected from several adjacent cells in multidimensional flow, the computational procedure determines which neighboring cells contribute to the moment calculations and then computes the new values for each cell. Thus the moment distributions are calculated using

$$
\begin{gathered}
c^{n+1}=\sum C_{m} \\
F^{n+1}=\frac{\sum C_{m}{ }^{n}}{C^{n+1}} \\
\left(R_{i}^{n+1}\right)^{2}=\frac{\sum c^{n}(\S i) R_{i}^{n^{2}}}{C_{i}^{n+1}}+12\left[\frac{\sum c^{n}(\S i) F_{i}^{n^{2}}}{C_{i}^{n+1}}-\left(\frac{\sum C^{n}(\xi i) F_{i}^{n}}{C_{i}^{n+1}}\right)^{2}\right]
\end{gathered}
$$

where $n+l$ denotes values at the new time step and $m$ denotes cell location in the computational mesh. Equation 23 is obtained from the modified second-moment algorithms derived by Prahm and Pedersen 51 .

The diffusion terms in Equation 1 are solved with a forwardin-time, centered-in-space difference technique modified to account for variable grid spacing. In this study, the horizontal mesh distance was equally spaced while the vertical mesh spacing was varied to account for regions where variables change rapidly with height. The concentration within a cell acted upon by vertical diffusion is calculated by the equation

$$
c_{k}^{n+1}=\left(1-\alpha_{k+1}-\alpha_{k-1}\right) c_{k}^{n}+\alpha_{k+1} c_{k+1}^{n}+\alpha_{k-1} c_{k-1}^{n}
$$

where $k$ denotes concentration at the vertical level, $k$. The horizontal subscripts for $x$ and $y$ directions have been deleted. The values $\alpha_{k+1}$ and $\alpha_{k-1}$ are defined as 


$$
\begin{gathered}
\alpha_{k+1}=\frac{\left(k_{z_{k}}+k_{z_{k-1}}\right) \Delta t}{\Delta z_{k}\left(\Delta z_{k}+\Delta z_{k+1}\right)} \\
\alpha_{k-1}=\frac{\left(k_{z_{k}}+k_{z_{k-1}}\right) \Delta t}{\Delta z_{k}\left(\Delta z_{k}+\Delta k_{k-1}\right)}
\end{gathered}
$$

where the diffusion coefficients are evaluated at the center of the grid mesh and $\Delta z_{k}$ denotes the depth of the $k^{\text {th }}$ grid. At the top and bottom boundary cells, the a's are defined as

$$
\left.\begin{array}{l}
\alpha_{k+1}=\left(1-r_{\text {top }}\right) \alpha_{k-1} \\
\alpha_{k-1}=\left(1-r_{\text {bottom }}\right) \alpha_{k+1}
\end{array}\right\}
$$

where $r$ varies between 0 and 1 to account for absorption or loss of material from the grid network ( $\left.\mathrm{Kao}^{41}\right)$. In order to maintain conservation of the zeroth, first, and second moment distribution following solution of the diffusion terms, the first and second moments are recalculated using the following general relations (for one-dimensional vertical diffusion):

$$
\begin{aligned}
F_{k}^{n+1} & =\left[C_{k}^{n} F_{k}^{n}\left(1-\alpha_{k+1}-\alpha_{k-1}\right)+\alpha_{k+1} C_{k+1}^{n}+F_{k+1}^{n}\right. \\
& \left.+\alpha_{k-1} C_{k-1}^{n} F_{k-1}^{n}\right] / C_{k}^{n+1} \\
\left(R_{k}^{2}\right)^{n+1} & =\left\{c_{k}^{n}\left[\left(R^{2}\right)_{k}^{n}+12\left(F_{k}^{n}-F_{k}^{n+1}\right)^{2}\right]\left(1-\alpha_{k+1}-\alpha_{k-1}\right)\right. \\
& +\alpha_{k+1} C_{k+1}^{n}\left[\left(R^{2}\right)_{k+1}^{n}+12\left(F_{k+1}^{n}+F_{k+1}^{n+1}\right)^{2}\right] \\
& +\alpha_{k-1} C_{k-1}^{n}\left[\left(R^{2}\right)_{k-1}^{n}\right. \\
& \left.\left.+12\left(F_{k-1}^{n}-F_{k-1}^{n+1}\right)^{2}\right]\right\} / C_{k}^{n+1}
\end{aligned}
$$


Similar expressions are used for the horizontal and lateral moments as well.

In order to enhance resolution of the peak concentration and prevent inaccurate horizontal spread (due to computational damping), a slightly modified version of the second moment solution is used. Based on the technique developed by Pedersen and Prahm51, a width correction procedure is used to check the lateral spread of concentration with in each cel1. If $R_{m}{ }^{n+1}$ is greater than $F_{m}{ }^{n+1} \pm 0.5$, then $R_{m}{ }^{n+1}=\min \left|F_{m}{ }^{n+1}+0.5\right|$. This procedure reduces the smal1 amounts of lateral dispersion errors produced when the concentration field is nonuniform and the flow field irregular. An example of the effect of width correction on the second-moment method under variable conditions is given by Pepper and Long 48 .

The computational domain consists of 2640 cells, 30 cells in the longitudinal direction $(x), 11$ cells in the lateral direction $(y)$, and 8 levels in the vertical direction $(K)$. Equal mesh spacing is used inn the horizontal plane with $\Delta x=\Delta y=2000 \mathrm{~m}$. The vertical mesh is given as

$$
\begin{aligned}
& z_{1}=0 \\
& z_{2}=2 \mathrm{~m} \\
& z_{3}=350 \mathrm{~m} \\
& z_{4}=1000 \mathrm{~m} \\
& z_{k}=z_{k-1}+\left(H-z_{k}\right) / 4
\end{aligned}
$$

where the height of the anvil, $\mathrm{H}$, used in this study is $15,000 \mathrm{~m}$. The height of the cloud base above the ground is set to $1000 \mathrm{~m}$, corresponding to $z_{4}$. The time step increment, $\Delta t$, is equal to $30 \mathrm{sec}$. This insures the stability criteria, $\lambda<\overrightarrow{\mathrm{U}} \Delta \mathrm{t} / \Delta \mathrm{s}$, where $\overrightarrow{\mathrm{U}}$ and $\Delta s$ correspond to the particular direction of the velocity vector and grid interval. The translational velocity of the cloud center is prescribed according to the translational speeds of the tornado, given in Table 1 .

The magnitudes of the values chosen for the initial distribution of concentration (Figure 3 ) are based on crude assumptions. A more realistic distribution may be achieved by incorporating the tornado model proposed by Fujita 6 into the existing model and calculating the uptake and injection of the concentration into the thunderstorm cell. Lack of a definitive severe storm model restricts calculation of the concentration once in the thunderstorm. However, additional work could conceivably provide insight into the initial dispersal patterns within the storm. 
A modified second-moment technique is again used to advect the updraft and downdraft velocity distribution at the $1000 \mathrm{~m}$ level (Figure 4 and Figure 5). The horizontal distribution of vertical velocity is advected and diffused according to parameters established within the three-dimensional concentration code. Incorporation of particulate fall velocities with $v_{g}=0.3 \mathrm{~m} / \mathrm{sec}$ is added to the vertical velocity field to account for the terminal fall velocity of $10-\mu$ plutonium particles. Within the thunderstorm cell, the terminal velocity of particles ranging from 1 to $10-\mu$ in radius is negligible compared to the updraft and downdraft velocities. If the rainout removal rate is 1 arge, particle deposition becomes negligible. Once the storm has passed and scavenging by rainout and washout is over, particulate deposition becomes more significant.

Rain is assumed to fall throughout the lifetime of the thunderstorm cell at a constant rate of $20 \mathrm{~mm} / \mathrm{hr}$. This assumption is obtained from the measured rainfall rates of tornadic storms analyzed by Fujita, et al.52. Heavier rainfall rates have the effect of increasing the depletion rate, resulting in deposition closer to the site. The $20 \mathrm{~mm} / \mathrm{hr}$ rate is felt to be the average value over the entire storm area.

The concentration is readjusted for rainout scavenging after calculation of the advection and diffusion terms in Equation 1 by the relation

$$
C_{k}^{n+1}=C_{k}^{n}\left[1-C_{1} \Delta t \frac{J_{0}}{R_{m}} E\left(a, R_{m}\right)\right]
$$

where the horizontal indices for the $x$ and $y$ locations of concentration have been deleted. The value of $C_{1}$ is equal to $0.5, R_{m}$ is the mean droplet diameter of the hydrometers within the cloud and is arbitrarily set, and $J_{o}$ is equal to $20 \mathrm{~mm} / \mathrm{hr}$. The values for $\mathrm{C}_{1}$ and $\mathrm{E}$ are derived by SIinn 53 based on data obtained by Burtsev, et al., 54 for removal rates in convective storms.

Updrafts markedly reduce the effects of rainout in the forward portions of the storm cell. $\Psi$ is set equal to zero in those regions of the storm where vertical velocities are positive upwards. This allows the rainfall to occur in the trailing edges of the storm, corresponding to the rain core and downdraft regions observed in actual storms. In order for the model to maintain conservation of mass within the computational domain, the decrease in concentration in the upper levels of the thunderstorm cell due to rainout is counterbalanced by an equal increase in the surface concentration below the cloud. 
Computer storage is moderate due to the peripheral subroutine used to generate the three-dimensional wind fields and contour displays. Optimization of the basic program would help reduce the core requirements. Simulation of pollutant dispersal over one hour (corresponding to a longitudinal distance of approximately $60 \mathrm{~km}$ from the point of initial breachment of the nuclear facility), requires about two minutes of computer time on an IBM-360/195.

Listings of the computer programs have been sent to Dak Ridge National Laboratory, Argonne National Laboratory, and the Nuclear Regulatory Commission. The program is written in FORTRAN IV language for use on an IBM-360/195 computer.

\section{RESULTS}

Numerous cases were simulated to determine the most likely dispersion patterns as well as potential radiological hazard to the people. The results shown in this study should be regarded as conservative estimates.

Output of the numerical model consists of concentration values specified within individual cell volumes. These values are appropriately adjusted with in cell volume to correspond to the spatial dimensions of the cell. Since the amount of radioactive debris picked up by a tornado varies according to the structural damage sustained, a unit release of material has been used to specify the source term. Results are presented as isopleths of ground-level air concentration ( $r$ atio of concentration to source mass, $\left.X / Q, m^{-3}\right)$ and surface deposition $\left(\mathrm{m}^{-2}\right)$ at $t=60$ minutes. Centerline ground-level values of air concentration (maximum values) are shown as a function of longitudinal distance along the trajectory of the storm. The isopleths are drawn with respect to distance from the point where the material is initially dispersed with in the storm.

The convergence and divergence of the mesoscale wind field are not considered; therefore, the longitudinal wind transports the storm cell in a straight line. Since the direction of the tornadic storm is arbitrary, direction is independent of points of the compass. Similarly, since dispersion is a function of translational windspeed, the translational velocities are input into the model corresponding to each design-basis tornado. Lateral dispersion along the trajectory of the storm is due primarily to the horizontal extent of the downdraft region (and rotational wind field) in the rear of the storm with minor influence from horizontal diffusion. Scavenging acts to dilute the concentration in the cloud so that ground-level air concentrations are less than those obtained without scavenging. 
The initial air concentration distribution $(X / Q)$ is shown in Figure $8 a$ in the $x-z$ plane with the center of the plane passing through the axis of the tornado $(t=0)$. Figures $8 b$ and $8 c$ show air concentration $\left(\mathrm{m}^{-3}\right)$ isopleths for $\vec{U}=13.4 \mathrm{~m} / \mathrm{sec}$ in the $x-z$ plane at $t=5$ and $40 \mathrm{~min}$, respectively. Figure 9 shows groundlevel air concentration in the $x-y$ plane at $t=40 \mathrm{~min}$.

Ground-level centerline $X / Q$ values are shown in Figures 10 and 11 for each specific translational velocity. The displacement of concentration as a function of translational velocity is evident. In all three cases, $90 \%$ of the peak air concentration has reached ground-level with in one hour after initial dispersion within the cloud ( $20 \mathrm{~min}$ after uptake of the pollutant). The decrease of $X / Q$ values beginning at $X=20 \mathrm{~km}$ in Figure 11 is due to the depletion of concentration from the cloud (except that part transported to the anvil region) and to nearly complete diffusion of the concentration below the cloud base to the ground.

Isopleths of air concentration at ground level for $t=60 \mathrm{~min}$ are shown in Figure 12 corresponding to storm translational velocities of $\vec{U}=13.4,19.2$, and $25.0 \mathrm{~m} / \mathrm{sec}$, respectively. The irregularity in the isopleth contours is due primarily to the advection and diffusion of the concentration by the updraft/downdraft regions and rainout removal rate of the storm with time. The ground-level layer consists of unit cells with dimensions of $2000 \mathrm{~m} \times 2000 \mathrm{~m} \times 2 \mathrm{~m}$. Figure 12 shows that as the translational velocty of the storm increases, the lateral spread of air concentration is stretched downwind. Higher peak concentrations appear less displaced to the right for the tornadic storm with a translational velocity of $13.4 \mathrm{~m} / \mathrm{sec}$ than with the succeeding two velocities. However, once beyond the initial peak concentration area, downwind values of ground-level air concentration are less than values obtained for $\vec{U}=19.2$ and $25.0 \mathrm{~m} / \mathrm{sec}$. This is to be expected because the increase in advection causes the peak concentration values to be more displaced in the longitudinal direction. Similarly, the slower the translational velocity, the more effects turbulent diffusion, vertical advection, and rainout have to act on the airborne concentration.

In test cases run without the influence of updrafts, downdrafts, and scavenging, the air concentration eventually reached ground after six hours, but was several orders of magnitude less in value. If the storm moves at $25 \mathrm{~m} / \mathrm{sec}$ for six hours, deposi$t$ ion at the surface would begin approximately $540 \mathrm{~km}$ from the plant. However, studies made by Davis 8 , Hane ${ }^{9}$ and Jessup 10 indicate that it would be unlikely for the pollutant to remain entirely with-in a storm cell for several hours without vertical wind shear and scavenging bringing a fraction of the pollutant to the surface. 

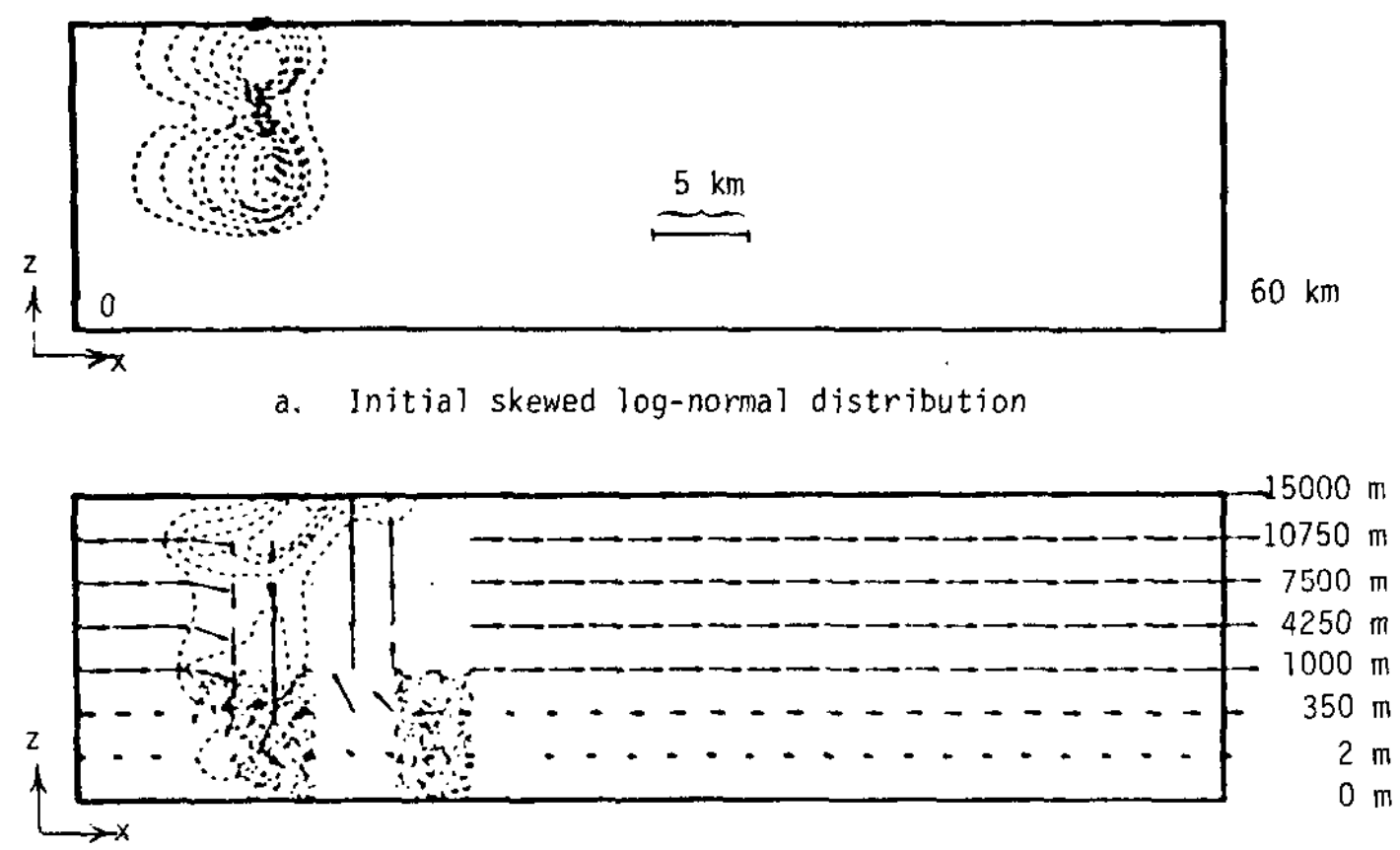

b. Air concentration at $t=5$ min (particles represent rain)

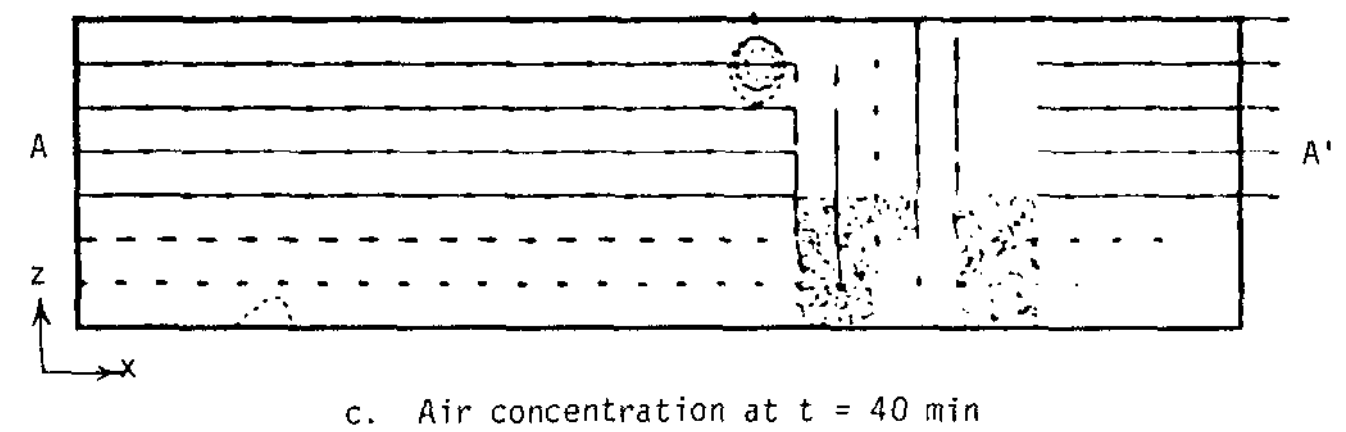

Figure 8. Air Concentration in the $X-Z$ Plane (Center of Plane Passing Through Axis of Tornado)

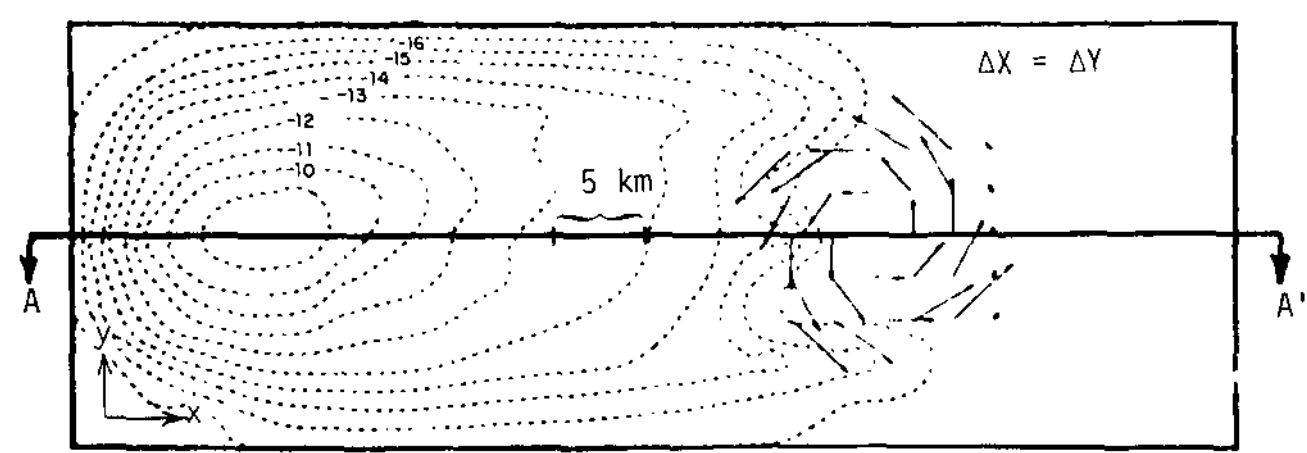

Figure 9. Ground Level Air Concentration in the X-Y Plane $(\mathrm{T}=40 \mathrm{~min}$ ) (Rotational Winds Represent Tornadic Storm) 


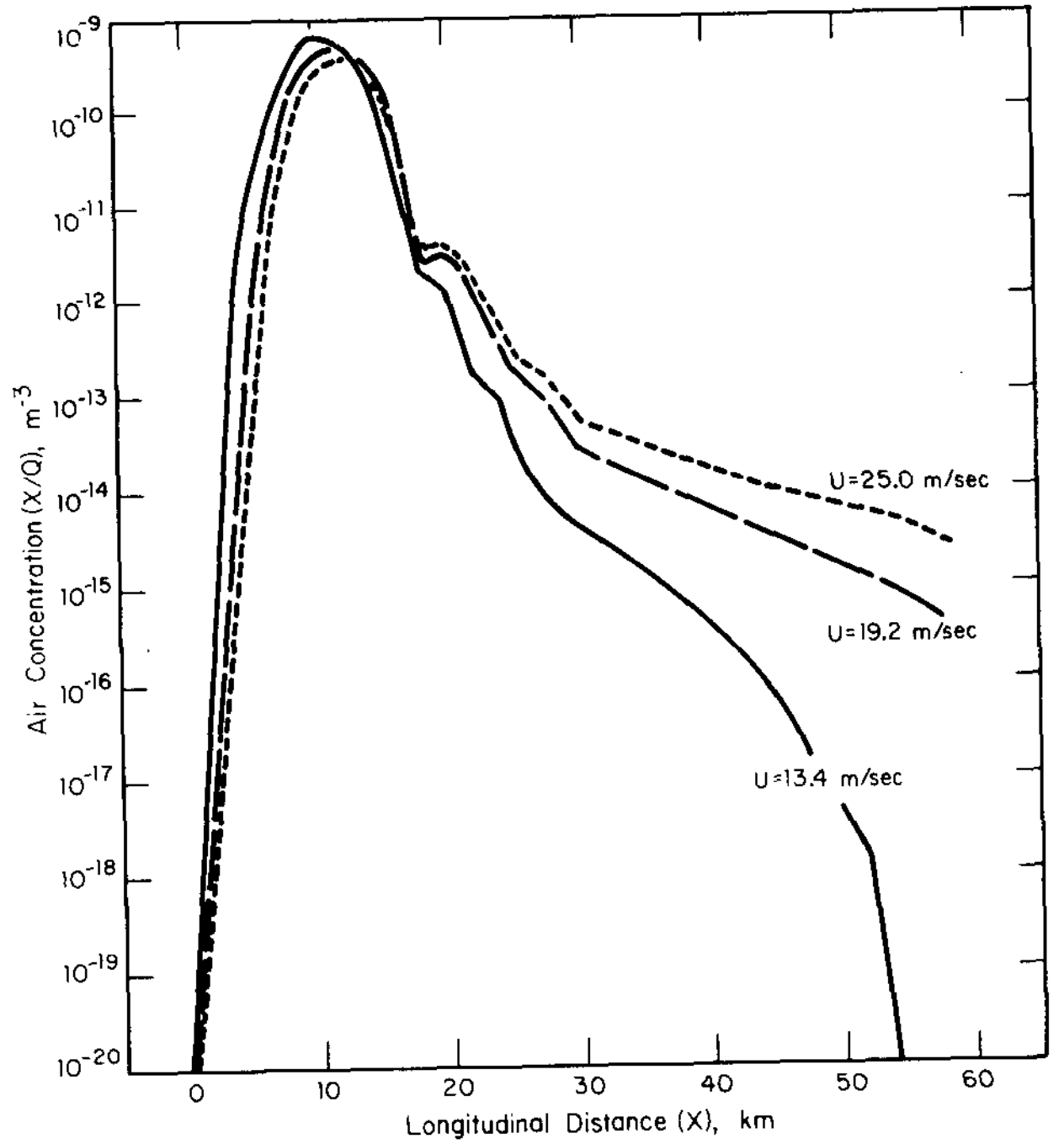

Figure 10. Maximum Ground-Level Centerline Air Concentration from Initialization Point in Storm 


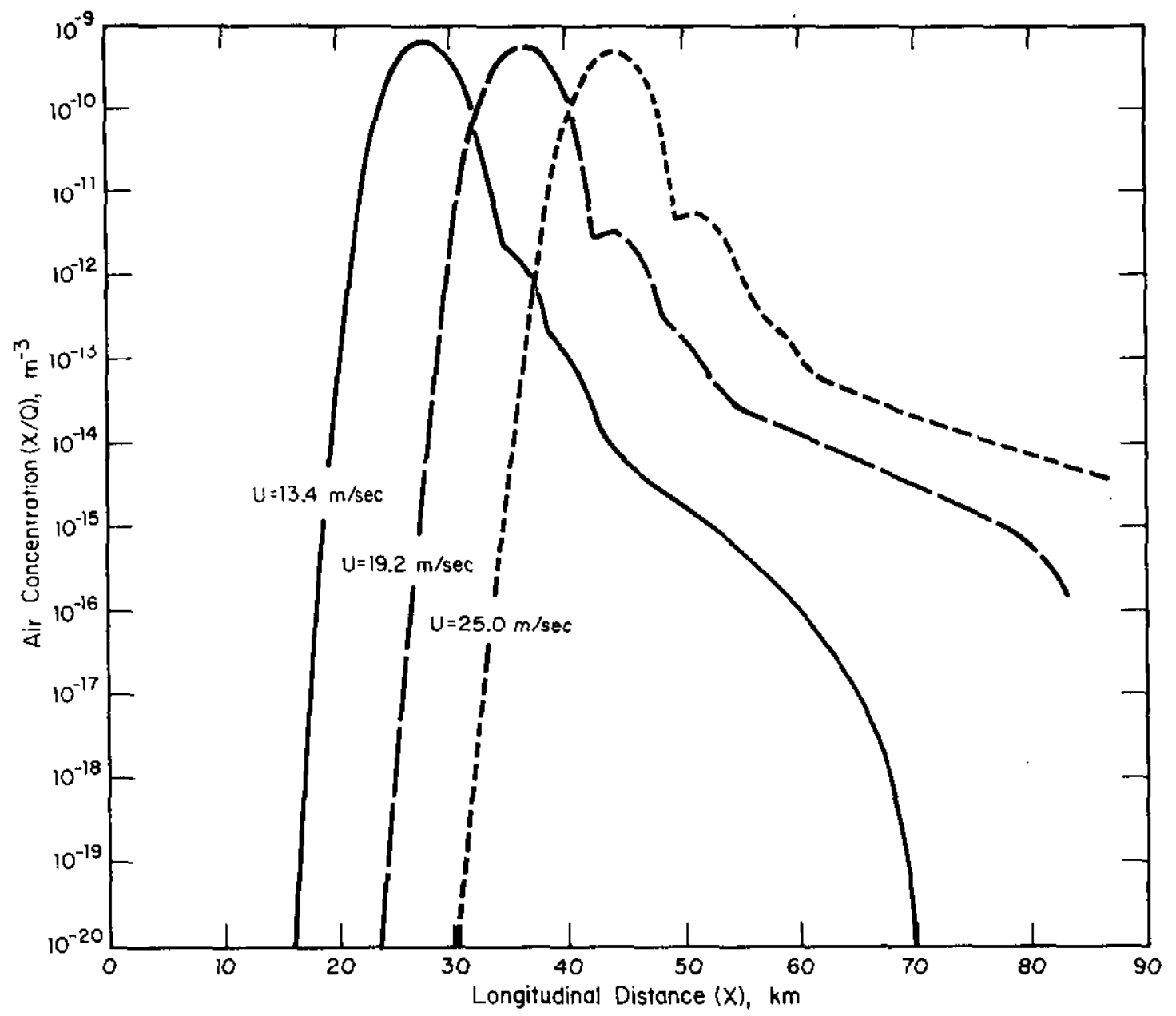

Figure 11. Maximum Ground-Level Centerline Air Concentration from Plant Site 

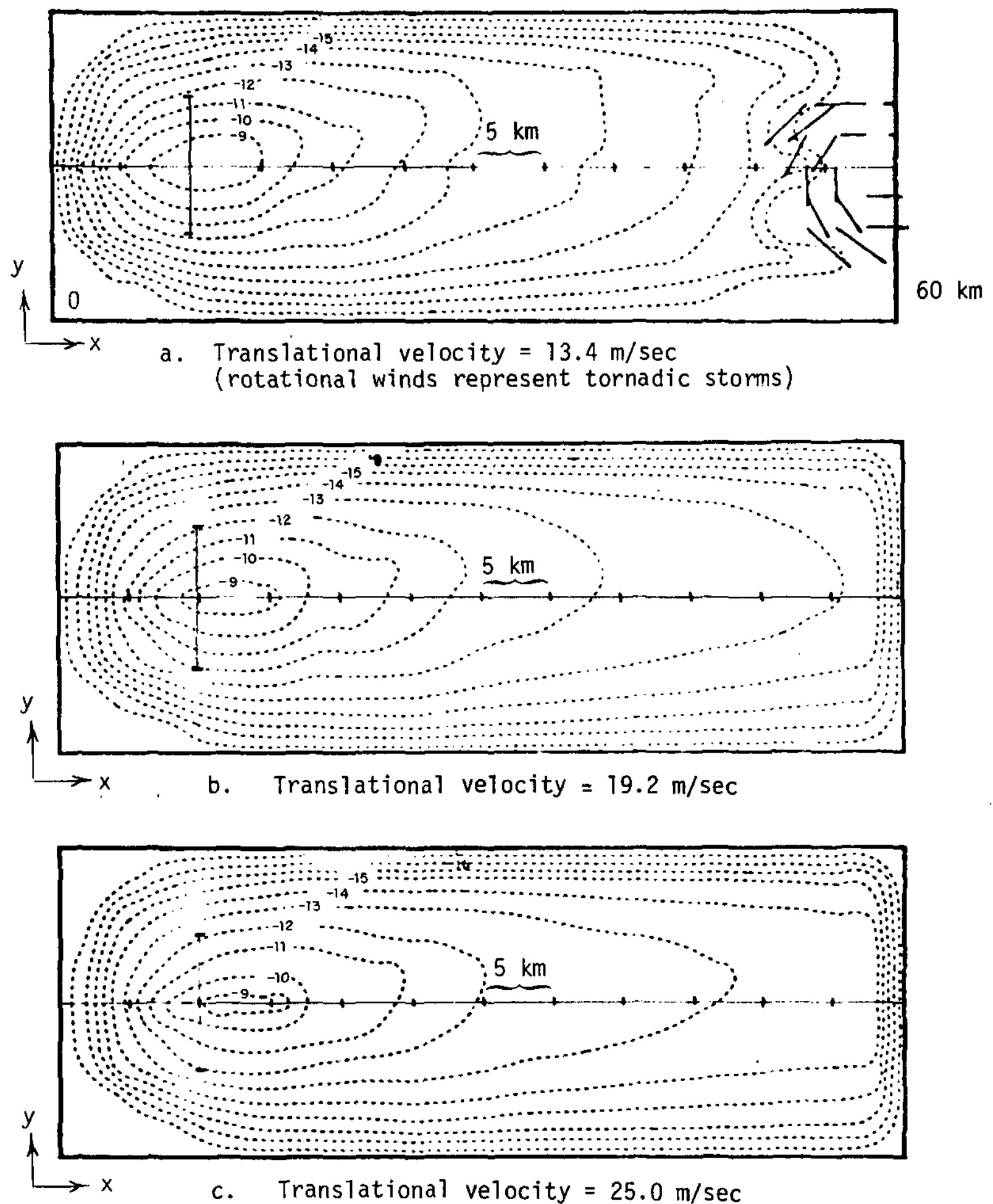

Figure 12. Ground-Level Air Concentration Isolog Plots $\left(\mathrm{m}^{-3}\right)$ in the $X-Y$ Plane $(T=60 \mathrm{~min})$ 
Ground-level raindrop depositions $\left(\mathrm{m}^{-2}\right)$ are shown in Figure 13 for $\vec{U}=13.4,19.2$, and $25.0 \mathrm{~m} / \mathrm{sec}$, respectively. The effect of advection on air concentration is also evident on groundlevel deposition: the highest peak values are obtained when $\vec{U}=13.4 \mathrm{~m} / \mathrm{sec}$ with the peak region being nearest to the initial dispersion point in the cloud; subsequent downwind values are less in value than the succeeding cases with $\vec{U}=19.2$ and $25.0 \mathrm{~m} / \mathrm{sec}$. The increase in translational velocity causes the region of peak concentration to be shifted along the direction of the storm.

Based on the test cases analyzed in this study, early deposition of concentration occurs within 10 to $20 \mathrm{~min}$ after the initial dispersion of concentration within the storm cell. The primary mechanisms for concentration reaching the surface come from the effect of the downdraft vertical velocity distribution and wet deposition. In all cases, $50 \%$ of the initial concentration, except that portion lifted into the anvil region of the cloud, is removed from the cloud within $15 \mathrm{~min}$ from the time of initial dispersion within the storm. The maximum ground-level concentration, in all cases occurs within about $35 \mathrm{~min}$ of ground-level injection.

Maximum centerline air concentration values reveal that peak concentration at the surface occurs within $15 \mathrm{~km}$ from the point where the initial dispersion within the storm is established (Figure 11). The concentration is essentially removed from the lower and middle layers of the cloud within $50 \mathrm{~km}$ of the peak ground-level value.

The modified Gaussian puff tornado model developed by Pepper ${ }^{7}$ was also used to calculate ground-level concentration and lateral spreading $\left(2 \sigma_{y}\right)$ of radioactive debris. Figure 14 shows the results of the Gaussian puff centerline values. The peak value occurs closer to the nuclear facility than that predicted by the numerical model. In these tests, the pollutant was diffused from a height of $6,500 \mathrm{~m}$ with an initial vertical distribution of $12,000 \mathrm{~m}$ $\left(\sigma z_{0}\right)$ and a lateral distribution of $5,000 \mathrm{~m}\left(\sigma y_{0}\right)$. These initial standard deviations were chosen to represent the material being dispersed within the thunderstorm cell. Calculation of the turbulent diffusion parameters (standard deviations) were based on a turbulent energy dissipation rate equal to $1 \mathrm{~m}^{2} / \mathrm{sec}^{3}$. Such values of energy dissipation rates are typical of the atmosphere associated with severe storms (Agee 35, Frisch and Strauch ${ }^{36}$ ).

The Gaussian puff model predicts ground-level concentration values that are several orders of magnitude less than those predicted by the numerical model. Similarly, the lateral spread of concentration at ground-level (distances obtained at $+2 \sigma y$ from the center of the puff) is significantly greater than that predicted by the numerical model. These rather small values of ground-level concentration are due primarily to the initial height at which the 


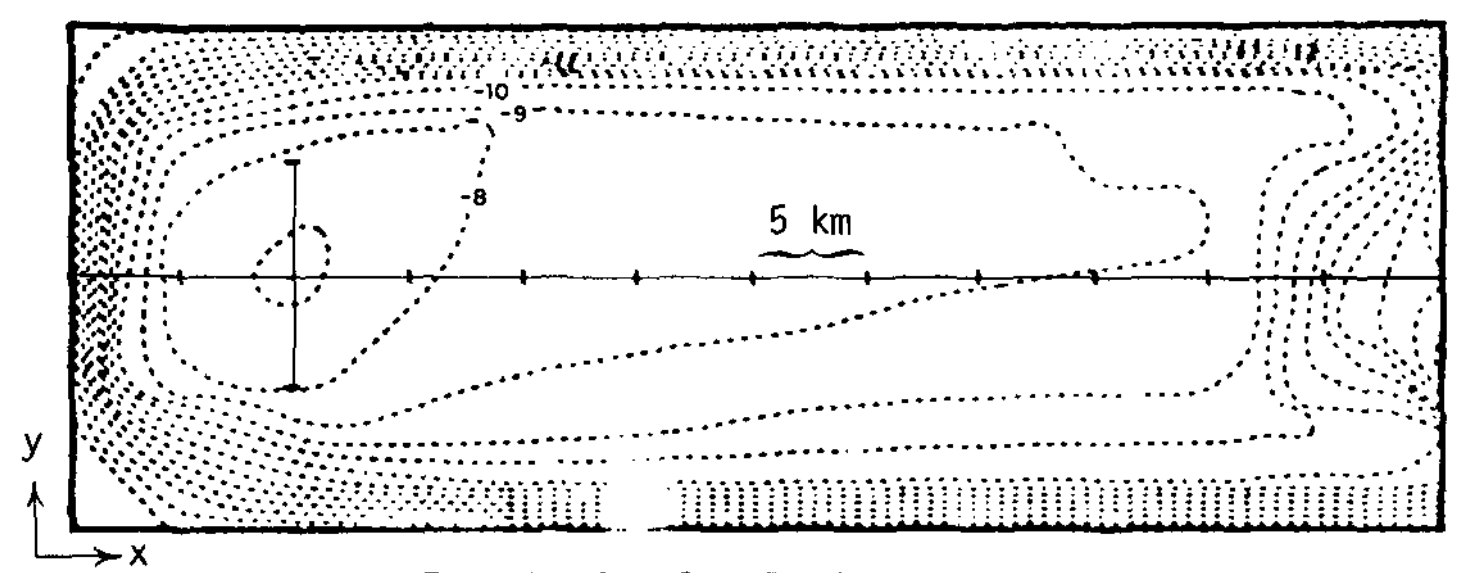

a. Translational velocity $=13.4 \mathrm{~m} / \mathrm{sec}$
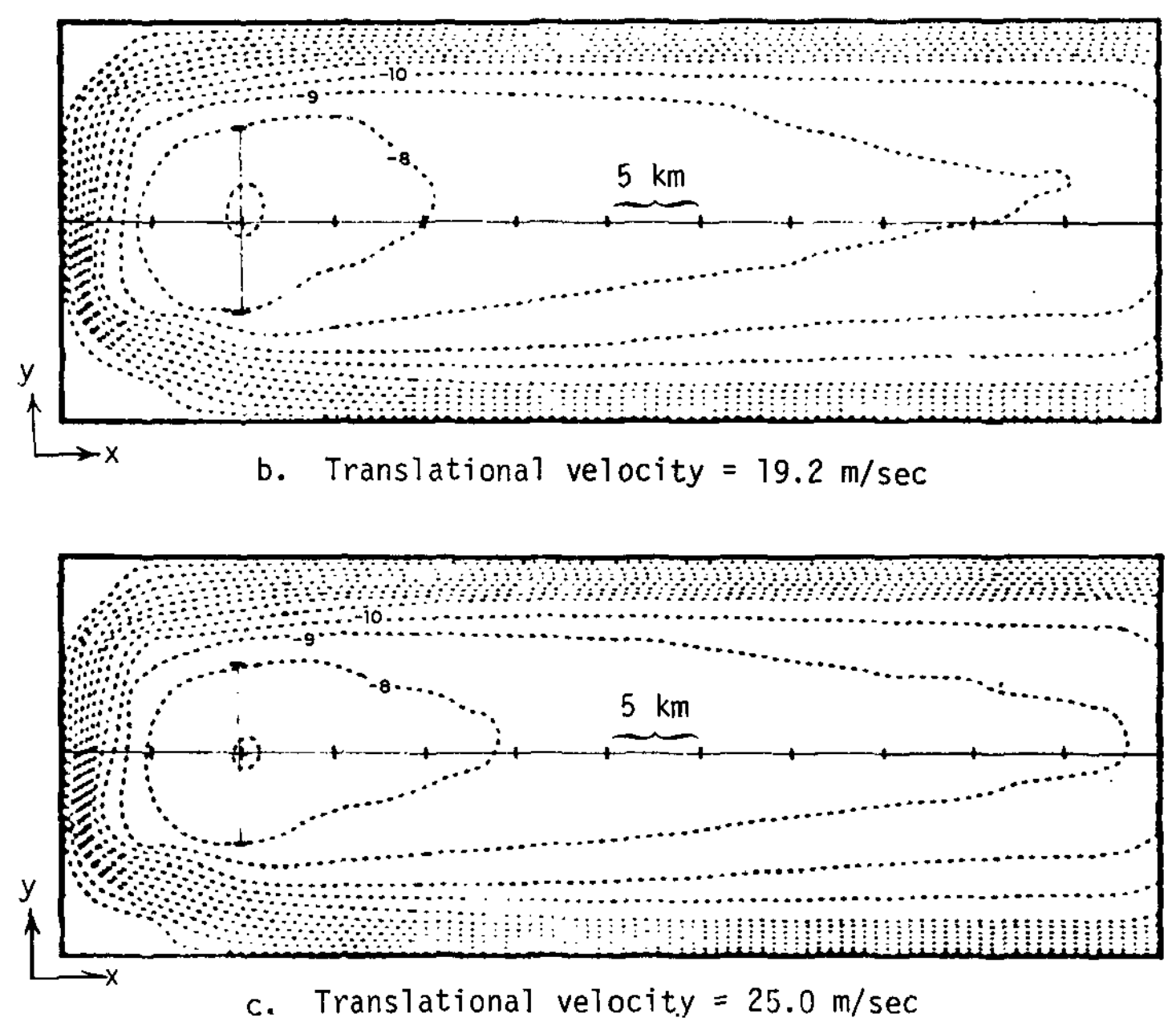

Figure 13. Ground-Level Deposition Isolog Plots $\left(\mathrm{m}^{-2}\right)$ in the $X-Y$ Plane $(T=60 \mathrm{~min})$ 


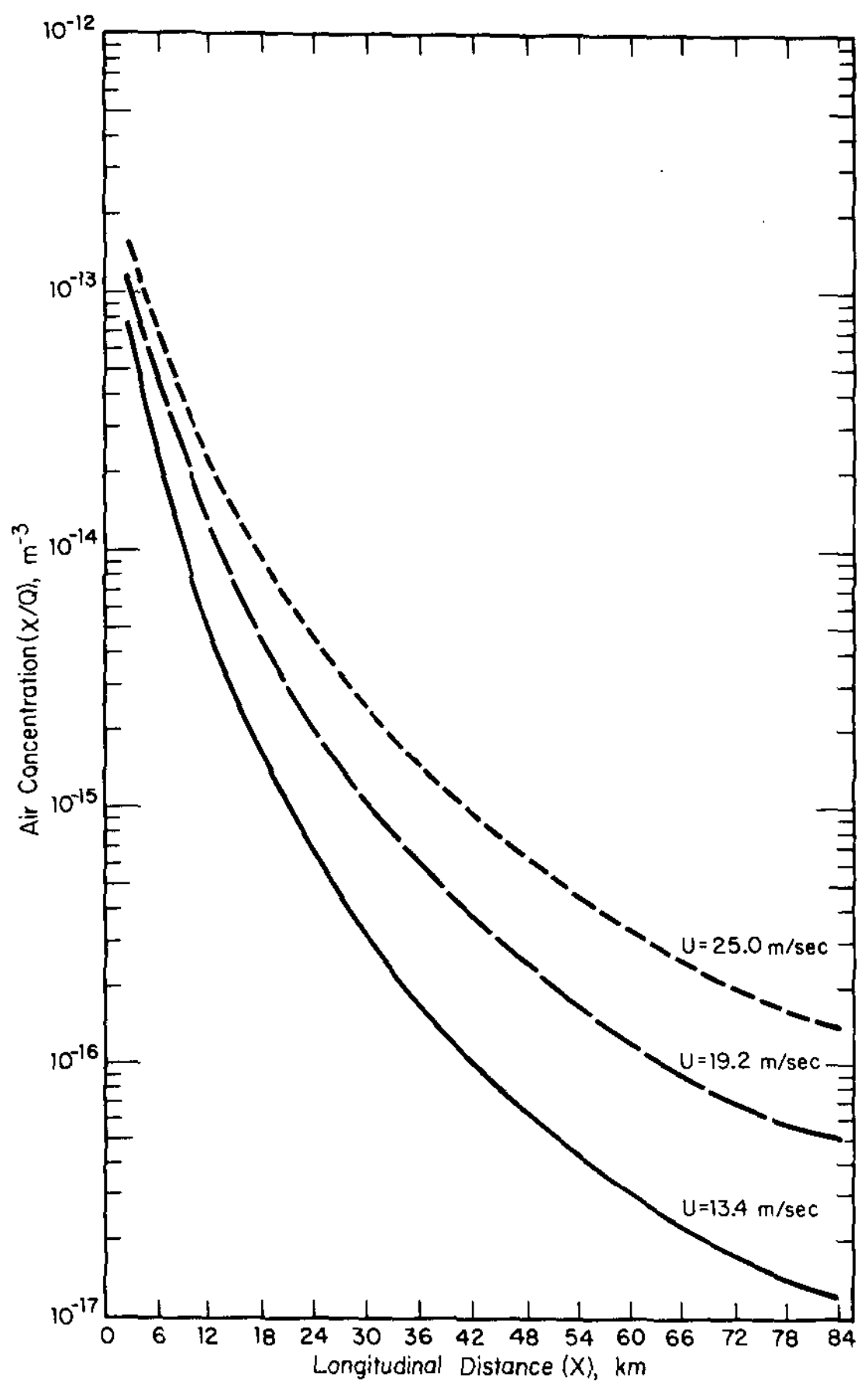

Figure 14. Maximum-Ground Level Centerline Air Concentration $\left(m^{-3}\right)$ - Modified Gaussian Model 
Gaussian model begins to diffuse the concentration, and to the high energy dissipation rate used to generate the diffusion parameters. A modified Gaussian model to include the effects of scavenging and topography ( $\mathrm{KaO}^{41}$ ) was also developed and the results compared to the Gaussian puff model developed by Pepper ${ }^{7}$. Only slight improvement was obtained in accounting for more rapid diffusion to the ground.

Figure 15 shows concentric annuli from the initial cloud dispersion point with radii of $1,2,3,4,5,6,7,8,9,10,20,30$, and 40 miles in $22.5^{\circ}$ sectors overlaid on the $x-y$ grid network. Average air concentration and deposition values after $60 \mathrm{~min}$ are given in Tables 3 and 4 corresponding to sector-averaged groundlevel values for each of the three translational velocities, respectively. Since the directional dependence of the storm has been eliminated, sector values for 180 to $360^{\circ}$ are considered to be zero. The centerline trajectory of the storm lies between Sectors 4 and 5 . Appropriate assignment of the centerline trajectory of the storm to a specific direction ( $N$, NNE, $E$, etc.), would then give corresponding sector averages based on compass points.

\section{MODEL SENSITIVITY}

\section{A. Effect of Neglecting Diffusion}

Most dispersion models neglect the effect of longitudinal diffusion since $u \partial c / \partial x$ is generally much larger than $\partial / \partial x\left(k_{x} \partial_{c} / \partial_{x}\right)$. In these cases where the source strength does not significantly vary with location, the lateral diffusion term is often neglected. The effect of neglecting horizontal dispersion generally increases as the wind velocity increases, with the transport becoming predominantly advection-oriented. When the winds are calm, diffusion becomes the dominant mechanism. However, in regions where the concentration gradients are very steep, horizontal diffusion cannot be neglected (when the concentration is undergoing rapid change).

Severe thunderstorms contain regions of intense updrafts and downdrafts and highly turbulent horizontal winds. Although the storm cell may be steered in a straight 1 ine, the storm cell fluid dynamics are very complex, with a continual cascade of turbulent energy from large- to small-scale eddies. It is reasonable to assume that the dispersion within the cloud is essentially advection-dominated. However, as the storm cell dissipates and the winds be come calm, lateral diffusion begins to spread the concentration in the horizontal plane. Tornadic storm cells have been observed to increase horizontally following dissipation of the tornado even though the intensity of the storm cell has decreased (Fujita, et al.52). Also, the decay of one storm cell has been known to be followed by generation of a new storm cell, 


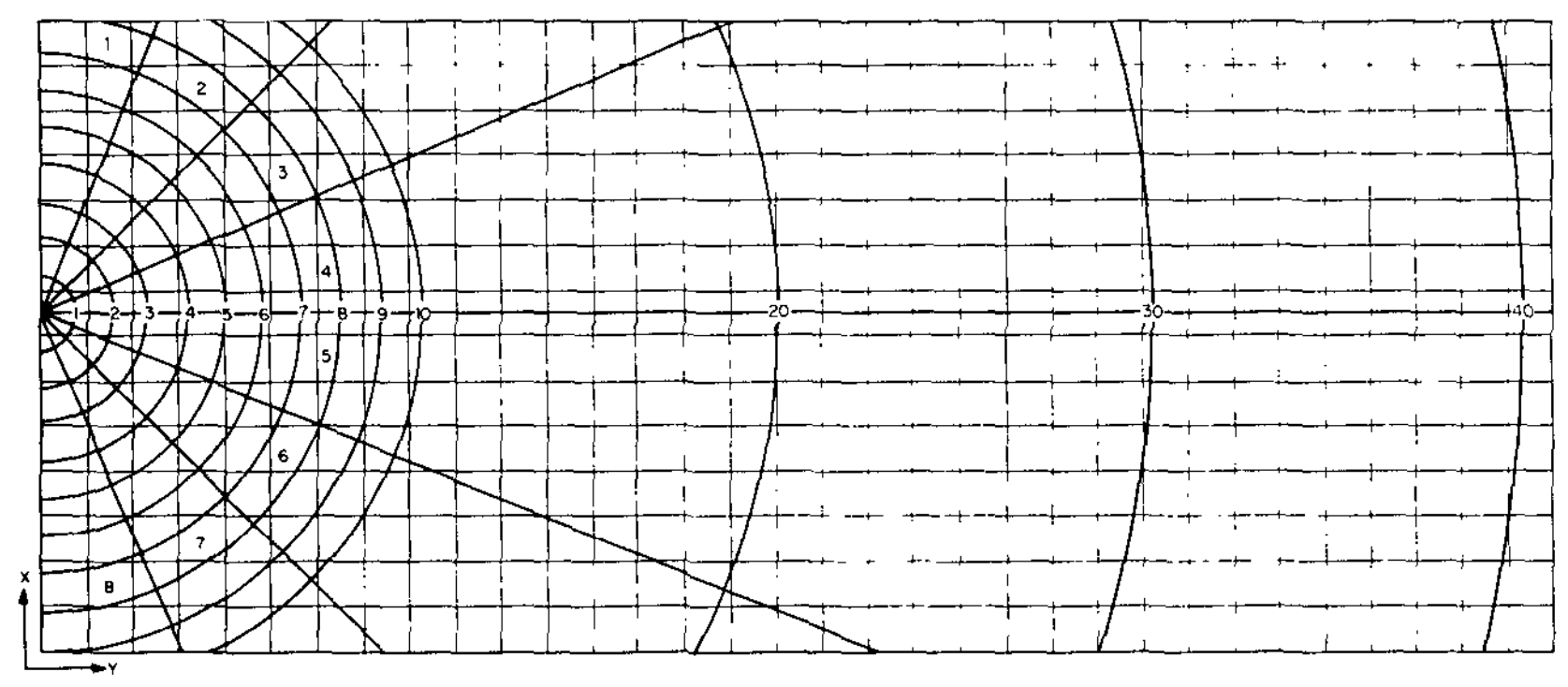

Figure 15. Concentric Annuli in $22.5^{\circ}$ Sectors in the X-Y Plane 
TABLE 3

Average Sector Air Concentration $\left(\mathrm{m}^{-3}\right)$ at Ground Level*

\begin{tabular}{|c|c|c|c|c|c|c|c|c|c|}
\hline ector & $\rightarrow \begin{array}{c}\text { TRANS } \\
\end{array}$ & VEL $=1$ & 4 & 4 & 5 & 6 & 7 & 8 & $\begin{array}{l}\text { Radius, } \\
\text { mile }\end{array}$ \\
\hline & $\begin{array}{l}0.0 \\
0.0 \\
0.0 \\
0.0 \\
0.0 \\
0.0 \\
0.0 \\
0.0 \\
0.0 \\
0.0 \\
0.0 \\
0.0 \\
0.0\end{array}$ & $\begin{array}{l}0.0 \\
7.3 E-14 \\
7.1 E-18 \\
4.0 E-16 \\
9.0 E-17 \\
5.0 E-18 \\
0.0 \\
2.8 E-20 \\
0.0 \\
0.0 \\
0.0 \\
0.0 \\
0.0\end{array}$ & $\begin{array}{l}0.0 \\
0.0 E-18 \\
1.1 E-15 \\
1.2 E-14 \\
2.0 E-13 \\
0.5 E-14 \\
4.0 E-14 \\
3.2 E-14 \\
2.0 E-15 \\
1.1 E-13 \\
9.4 E-17 \\
0.0 \\
0.0\end{array}$ & $\begin{array}{l}.0 \\
2.4 E-10 \\
3.3 E-15 \\
7.7 E-12 \\
5.7 E-11 \\
1.4 E-10 \\
2.4 E-10 \\
2.7 E-10 \\
1.0 E-10 \\
8.3 E-11 \\
1.2 E-11 \\
1.2 E-14 \\
1.0 E-17\end{array}$ & $\begin{array}{l}0.0 \\
2.4 E-16 \\
3.3 E-13 \\
0.0 E-12 \\
3.8 E-11 \\
1.0 E-10 \\
3.4 E-10 \\
3.5 E-10 \\
2.7 E-10 \\
1.5 E-10 \\
3.7 E-11 \\
4.0 E-14 \\
1.1 E-16\end{array}$ & $\begin{array}{l}0.0 \\
4.7 E-18 \\
1.2 E-15 \\
1.3 E-14 \\
3.0 E-13 \\
9.4 E-14 \\
1.7 E-13 \\
8.7 E-14 \\
2.3 E-14 \\
1.8 E-14 \\
2.4 E-15 \\
0.0 \\
0.0\end{array}$ & $\begin{array}{l}0.0 \\
4.2 E-19 \\
3.3 E-18 \\
4.0 E-16 \\
1.7 E-16 \\
1.8 E-16 \\
7.6 E-19 \\
6.5 E-19 \\
0.0 \\
0.0 \\
0.0 \\
0.0 \\
0.0\end{array}$ & $\begin{array}{l}0.0 \\
0.0 \\
0.0 \\
6.7 E-21 \\
1.5 E-20 \\
0.0 \\
0.0 \\
0.0 \\
0.0 \\
0.0 \\
0.0 \\
0.0 \\
0.0\end{array}$ & $\begin{array}{r}1 \\
2 \\
3 \\
4 \\
5 \\
6 \\
7 \\
8 \\
9 \\
10 \\
20 \\
30 \\
40\end{array}$ \\
\hline tor & $\begin{array}{c}\text { TRANS } \\
1 \\
\end{array}$ & $E L=1$ & 3 & 4 & 5 & 6 & 7 & 8 & $\begin{array}{l}\text { Radius, } \\
\text { mile }\end{array}$ \\
\hline & $\begin{array}{l}0.0 \\
0.0 \\
0.0 \\
0.0 \\
0.0 \\
0.0 \\
0.0 \\
0.0 \\
0.0 \\
0.0 \\
0.0 \\
0.0 \\
0.0\end{array}$ & $\begin{array}{l}0.0 \\
1.1 E-1 Y \\
1.1 E-18 \\
9.4 E-17 \\
2.1 E-17 \\
1.4 E-18 \\
0.0 \\
7.5 E-21 \\
0.0 \\
0.0 \\
0.0 \\
0.0 \\
0.0\end{array}$ & $\begin{array}{l}0.0 \\
1.2 E-18 \\
2 . c E-16 \\
2.5 E-15 \\
1.3 E-14 \\
2.5 E-14 \\
4.0 E-14 \\
2.2 E-14 \\
1.7 E-15 \\
1.1 E-15 \\
9.1 E-17 \\
0.0 \\
0.0\end{array}$ & $\begin{array}{l}U . U \\
2.2 E-10 \\
5.0 E-15 \\
1.1 E-12 \\
1.4 E-11 \\
4.1 E-11 \\
1.3 E-10 \\
1.0 E-10 \\
1.0 E-10 \\
9.4 E-11 \\
4 . U E-11 \\
\text { Y. OE - } 14 \\
\text { d. } 7 E-15\end{array}$ & $\begin{array}{l}0.0 \\
2.1 E-10 \\
5.0 E-15 \\
1.1 E-12 \\
1.4 E-11 \\
4.3 E-11 \\
1.0 E-10 \\
2.0 E-10 \\
1.0 E-10 \\
1.2 E-10 \\
0.7 E-11 \\
2.3 E-13 \\
1.8 E-14\end{array}$ & $\begin{array}{l}0.0 \\
6.1 E-15 \\
2.3 E-16 \\
2.7 E-15 \\
1.7 E-14 \\
1.8 E-14 \\
0.0 E-14 \\
3.5 E-14 \\
9.0 E-15 \\
7.5 E-15 \\
1.1 E-15 \\
0.0 \\
0.0\end{array}$ & $\begin{array}{l}0.0 \\
7.4 E-20 \\
0.4 E-19 \\
1.0 E-16 \\
4.6 E-17 \\
7.1 E-17 \\
3.0 E-19 \\
4.5 E-19 \\
0.0 \\
0.0 \\
0.0 \\
0.0 \\
0.0\end{array}$ & $\begin{array}{l}0.0 \\
0.0 \\
0.0 \\
2.1 E-21 \\
4.6 \mathrm{E}-21 \\
0.0 \\
0.0 \\
0.0 \\
0.0 \\
0.0 \\
0.1 \\
0.0 \\
0.0\end{array}$ & $\begin{array}{r}1 \\
2 \\
3 \\
4 \\
5 \\
6 \\
7 \\
8 \\
9 \\
10 \\
20 \\
30 \\
40\end{array}$ \\
\hline tor & $\begin{array}{r}\text { TKANS } \\
+\quad 1 \\
\end{array}$ & $\begin{array}{r}E L= \\
2\end{array}$ & 3 & 4 & 3 & 0 & 7 & 8 & $\begin{array}{l}\text { Radius, } \\
\text { mile }\end{array}$ \\
\hline & $\begin{array}{l}0.0 \\
0.0 \\
0.0 \\
0.0 \\
0.0 \\
0.0 \\
0.0 \\
0.0 \\
0.0 \\
0.0 \\
0.0 \\
0.0 \\
0.0\end{array}$ & $\begin{array}{l}0.0 \\
2.0 E-20 \\
1 . Y E-19 \\
2.4 E-17 \\
5.3 E-18 \\
5.1 E-19 \\
0.0 \\
3.0 E-21 \\
0.0 \\
0.0 \\
0.0 \\
0.0 \\
0.0\end{array}$ & $\begin{array}{l}0.0 \\
2.2 E-19 \\
5.8 E-17 \\
0.7 E-16 \\
4.7 E-15 \\
1.1 E-14 \\
2.5 E-14 \\
1.3 E-14 \\
1.2 E-15 \\
0.7 E-10 \\
0.9 E-17 \\
0.0 \\
0.0\end{array}$ & $\begin{array}{l}0.0 \\
4.0 E-19 \\
4.3 E-10 \\
2.0 E-13 \\
1.0 E-12 \\
1.2 E-11 \\
7.7 E-11 \\
1 . \angle E-10 \\
1.2 E-10 \\
8 . \angle E-11 \\
4.9 E-11 \\
2.0 E-13 \\
3.4 E-14\end{array}$ & $\begin{array}{l}0.0 \\
4.6 E-19 \\
4.0 E-10 \\
2.0 E-13 \\
3.0 E-12 \\
1.2 E-11 \\
7.7 E-11 \\
1.2 E-10 \\
1.2 E-10 \\
0.5 E-11 \\
0 . \angle E-11 \\
4.0 E-13 \\
0.5 E-14\end{array}$ & $\begin{array}{l}.0 \\
2.0 E-14 \\
0.6 E-17 \\
7.7 E-16 \\
5.9 E-15 \\
1.4 E-14 \\
3.0 E-14 \\
1.0 E-14 \\
5.1 E-15 \\
4.2 E-12 \\
7.1 E-16 \\
0.0 \\
0.0\end{array}$ & $\begin{array}{l}0.0 \\
1.8 E-20 \\
1.7 E-19 \\
3.1 E-17 \\
1.5 E-17 \\
3.1 E-17 \\
1.3 E-19 \\
3.2 E-19 \\
0.0 \\
0.0 \\
0.0 \\
0.0 \\
0.0\end{array}$ & $\begin{array}{l}0.0 \\
0.0 \\
0.0 \\
8.5 E-22 \\
1.9 E-21 \\
0.0 \\
0.0 \\
0.0 \\
0.0 \\
0.0 \\
0.0 \\
0.0 \\
0.0\end{array}$ & $\begin{array}{r}1 \\
2 \\
3 \\
4 \\
5 \\
6 \\
7 \\
8 \\
9 \\
10 \\
20 \\
30 \\
40\end{array}$ \\
\hline
\end{tabular}

: Values followed by the letter $E$ (for exponent), minus symbol, and two digits indicate the powers of 10 by which the number must be multiplied to obtain the correct value: for example, $7.3 \mathrm{E}-19$ is $7.3 \times 10^{-19}$. 
TABLE 4

Average Sector Deposition $\left(\mathrm{m}^{-2}\right)$ at Ground Level*

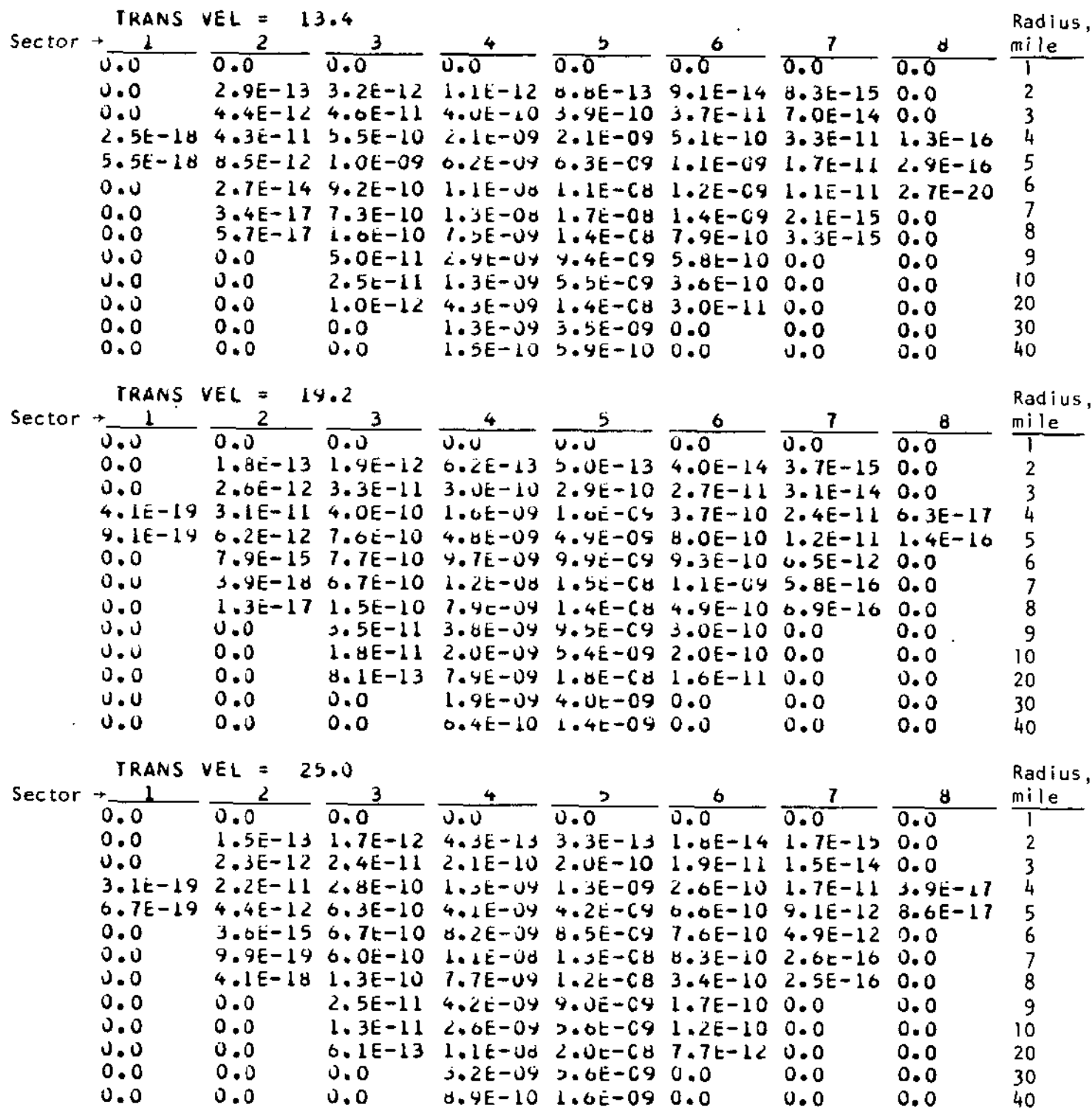

*Values followed by the letter $E$ (for exponent), minus symbol, and two digits indicate the powers of 10 by which the number must be multiplied to obtain the correct value; for example, $7.3 \mathrm{E}-19$ is $7.3 \times 10^{-19}$. 
with ingestion of the pollutant concentration possible in the new ce11. If this were to occur, it is assumed that the dimensions of the new thunderstorm cell would define the lateral boundaries of the concentration. Within the thunderstorm cell, the concentration would be continually sheared and dispersed by the winds.

Vertical diffusion is quite sensitive to the atmospheric temperature gradient. Highly unstable conditions create a turbulent flow field which in turn causes the pollutant to be well-mixed in the atmosphere. As the atmosphere becomes stable, the turbulence becomes suppressed, the winds become calm, and the pollutant is generally confined near the surface of the earth under an inversion. Because the tornadic storm is associated with extremely unstable conditions (within the thunderstorm cell), the pollutant is assumed to be well-mixed throughout the vertical extent of the cell. Below the base of the thunderstorm, the atmosphere is also unstable, but to a much lesser extent. In test cases run with only horizontal advection and vertical diffusion, the pollutant began to diffuse below the base of the cloud after two hours, but failed to reach ground level even after six hours. Abnormally high values of $\mathrm{k}_{z}$ did not significantly decrease the amount of time for the pollutant to reach the ground. This indicated that the dominant mechanism of pollutant dispersion was the advection terms. Hence the only way the pollutant could reach the ground in a short length of time was by downdraft and in-cloud scavenging. However, if the storm cell were to quickly dissipate, the vertical diffusion would become more significant as the wind speeds decrease in intensity.

\section{B. Effects of Wind Profiles}

The variation of the wind speed with height and magnitude significantly influence the downwind spread and shear of the pollutant cloud. The greater the wind speed, the further the pollutant is advected before settling to the ground. If the wind velocity increases dramatically with height, the concentration becomes sheared and distributed more longitudinally in the direction of the shear. As the wind profile becomes flat and the speed drops, vertical diffusion acts to spread the concentration throughout the mixed layer.

\section{Effects of Updrafts and Downdrafts}

Significant updrafts and downdrafts of tens of meters per second are known to exist in severe storms. The design bas is tornado used in this study is assumed to entrain radioactive particles ranging in size from $1-10 \mu$ into its vortex and then to lift the particles to the base of the storm cell. Once the particles enter the storm cell, the action of the severe updrafts 
and downdrafts act to disperse the particles throughout the storm cell. The updrafts 1 ift these small particles considerable distances, possibly out the top of the anvil and into the stratosphere. Downdrafts near the rear of the storm cause the pollutant to be dispersed below the storm cell. Test cases were run with and without the vertical wind vectors. Results showed that the concentration remained in the cloud over considerable lengths of time (hours) before turbulent diffusion brought the concentration to the ground. Inclusion of updrafts and downdrafts diluted the concentration in the vertical plane initially, but then began to bring the concentration to the ground much more rapidly.

\section{In-Cloud Scavenging (Rainout)}

Precipitation-scavenging field experiments by the Illinois State Water Survey and Batelle Northwest Laboratories have shown that scavenging by storms which are greater than 10,000-ft deep deposit a significant fraction of released tracer. However, in the study of dispersion with in tornadic storms, the following factors must be considered:

- Is the particulate incorporated entirely into the rain?

- Are there motions in the rain area which are upward (creating scavenging by consideration processes)?

- Does the rain area extend over the entire thunderstorm cell as the tornado and severity of the thunderstorm decrease?

These questions $c$ an be answered more accurately when more data becomes available. The present model assumes the concentra$t$ ion is acted upon by rainout and washout scavenging, except for that concentration which is lifted into the anvil (top level of the mode1). In test cases run with and without rainout, inclusion of the rainout sink term in the governing equations significantly added to early deposition of the radioactive particulate on the ground. Coupling of this effect with the effect of downdraft velocities within the storm account for earlier deposition than with downdraft velocities alone. The magnitude of the rainfall rate and mean droplet diameter determine the rainout removal rate. Under actual conditions, the rainfall rates vary throughout the lifetime of the storm and are more intense in some areas than in others. This in turn ultimately affects the deposition patterns on the ground, creating areas of intense concentration beneath the storm.

Since the radioactive material is assumed to be totally entrained within the thunderstorm, scavenging by washout is less significant than rainout scavenging. Should part of the pollutant 
be thrown out of the vortex below the base of the thunderstorm, washout is likely to contribute to early deposition near the strike point.

\section{E. Height of the Storm}

Severe tornadoes are generally associated with large singlecell convective storms with anvil tops reaching as high as $15,000 \mathrm{~m}$ or more. A tornadic storm with a $15,000 \mathrm{~m}$ top is considered to be typical of storms observed in the vicinity of the Pennsylvania plant site. The heights to which the concentration is initially assumed to reach are critical to the dispersion pattern. The higher the concentration is initally transported, the longer it takes for the concentration to be deposited on the ground. For point sources released at varying heights under less severe atmospheric conditions, downwind ground-level concentrations reach peak levels at greater distances from the initial point of release than those emitted closer to the ground. Turbulent mixing distributes the concentration vertically so that by the time the concentration begins to reach the ground, it has been sufficiently diluted. Evidence of radar signals of tracers in convective clouds indicate that wide-level dispersion occurs almost immediately following injection. The heights to which the concentration is reached in th is model is based on estimates made by Fujita* and Carson.** A compact distribution of concentration (puff) near the base of the thunderstorm cell, as used by Pepper ${ }^{7}$, could cause the concentration to be more quickly deposited near a nuclear facility in higher amounts.

\section{F. Effect of Topography (Surface Roughness Length)}

The influence of topography on a velocity vector field is well known from elementary fluid dynamics. In regions where the terrain is irregular, the flow fields converge and diverge in an effort to conform to the boundary shape of the terrain. Atmospheric flow over hills and mountains creates upward motion and wake-recirculation regions which are downwind of high terrain features. Since the advection of the wind strongly governs the dispersion pattern of concentration in a severe storm, accurate prediction of the wind velocities should give a fairly accurate estimation of the dispersion pattern. In regions where the terrain is essentially flat, classical 'flat plate' boundary layer analys is is usually adequate for determining the wind velocities. When the texrain is irregular, however, the equations of fluid motion must be solved in order to account for the secondary

\footnotetext{
* T. T. Fujita, Personal Communication, 1977.

** J. Carson, Personal Communication, 1977.
} 
motions created by the terrain. The effect of topography (surrounding the Pennsylvania plant site) on advection has not been considered in this study. However, the model can be modified to account for terrain by using a grid network with variable mesh spacing (Pepper and Baker 31 ). Provided the terrain features are known over the entire surface domain of calculation, and a mass-consistent wind field is generated (which requires reasonably good initial estimates of the three-dimensional flow field within the storm and its surrounding vicinity), the terrain influence on the deposition pattern can be taken into account. Neglect of the irregularity in terrain undoubtedly produces some degree of error in the calculations. As knowledge of the correlation between terrain feature and air flow patterns in severe storms improves, this effect can be more accurately modeled.

Topography also influences the degree of turbulence intensity which acts on the diffusion parameters in the atmosphere. Little is known about turbulent diffusion in severe storms, and even less about the effect of terrain on dispersion during severe storms. In order to account for the influence of terrain on the vertical diffusion coefficient, $\mathrm{K}_{\mathrm{z}}$, an empirical relation has been used which incorporates a surface roughness parameter, $z_{0}$. Turbulent diffusion below the base of the thunderstorm cell and near the surface is determined by an extrapolated surface layer of "lay of the wall" formulation which includes $z_{0}$. The higher the value of $z_{0}$, the more turbulent the diffusion near the surface. This empirical relation coupled with an additional relation for $\mathrm{K}_{z}$ above the surface layer allows the influence of surface roughness (or topography) to be propogated with height to the base of the thunderstorm ce11. The intensity of turbulence within the thunderstorm cell is felt to be significantly greater than that below the cloud base. Hence the influence of terrain on dispersion within the thunderstorm cell is considered to be negligible.

\section{CONCLUSIONS}

A three-dimensional numerical model is used to calculate the dispersion of small particles in a tornadic storm. The model is designed to allow various meteorological parameters to be updated as more precise information becomes available. The threedimensional transient equation of concentration transport is solved by a quasi-Lagrangian method of second moments in an Eulerian mesh centered over the assumed trajectory of the storm.

The horizontal wind field varies with height over the onehour period after the facility is breached. The updrafts and downdrafts associated with the tornadic storm are calculated from initial empirical estimates and then advected with the storm. The horizontal rotational wind field within the storm cell is also 
advected with the vertical velocity field. As the storm cell spreads horizontally, the wind field within the storm cell spreads accordingly.

Because of the lack of precise information regarding turbulence within severe storms, the turbulence diffusion coefficients are obtained from empirical estimates. These estimates are based on data measured within storms, theoretical equations appearing in the literature, and discussions with noted authorities on turbulence diffusion.

Scavenging is calculated as a sink term in the governing equation. Rainout scavenging acts on small particles within the cloud. However, limited knowledge of scavenging in severe storms necessitates the use of a simple general expression based on rainfall rates, droplet size, and $100 \%$ collision efficiency. Washout scavenging below the cloud base is not considered because of the high values of downdraft velocities associated with the storm. The effect of droplet evaporation is also neglected.

The effect of topography downwind of the plant is introduced through specification of roughness heights used in determining turbulent diffusion below the cloud. The effect of topography on advection is not considered.

The dispersion patterns and total time of uptake within the vortex have not been considered. However, the recent appearance of an engineering design-basis tornado model provides the threedimensional wind patterns surrounding the vortex. Inclusion of this model with the dispersion model should allow the trajectories and dispersion patterns of material to be calculated inmediately following ground-level uptake. In this study the pollutant is assumed to be dispersed throughout the thunderstorm cell in a skewed log normal distribution with the maximum value centered on the axis of the tornado. The material is allowed to disperse for $20 \mathrm{~min}$ before rainout contributes to the deposition. About $35 \%$ of the material is assumed to be dispersed with in the upper regions of the cloud, $15 \%$ within the middle section of the storm, and $50 \%$ with in the lower layers and cloud base of the storm.

The updraft and downdraft vertical velocity distributions and wet deposition account for most of the material being deposited at the surface one hour after initial uptake of the material. Scavenging accounts for about $50 \%$ of the particle removal from the cloud within $15 \mathrm{~min}$ (following the initial $20 \mathrm{~min}$ ).

In test cases without the inclusion of rainout and updrafts and downdrafts, the material diffuses to the ground after six hours following uptake into the cloud. Since the advection 
velocities dominate the dispersion of the pollutant, the effect of turbulent diffusion between the cloud base and the surface are not significant until the storm cell has traveled some distance. Although rainfall $r$ ates and droplet diameters vary during the life of the storm, a constant rainfall rate of $20 \mathrm{~mm} / \mathrm{hr}$ and a mean droplet diameter of $1 \mathrm{~mm}$ were chosen as ensemble averages in order to simplify the complexity of input data. Once the pollutant is completely dispersed with in the thunderstorm cell, capture of the particulates by rain drops begins immediately with 100 percent efficiency. The deposition of concentration at the surface consists primarily of plutonium particles suspended within waterdrops. As additional information on rainfall rates and velocities in tornadic storms becomes available, deposition will likely become high ly nonuniform.

Ground-level air concentration begins to reach the surface within $5 \mathrm{~min}$. Results show that ground-level concentrations begin occurring within 20 to $45 \mathrm{~km}$ from the plant site. Peak centerline concentrations occur within $15 \mathrm{~km}$ of the point of initial dispersion within the cloud. The concentration decreases significantly with distance after peak-ground level values are reached. The lateral spread of ground-level concentration is principally governed by the size of the thunderstorm cell directly overhead. Downdrafts and scavenging have more influence on bringing the concentration directly from the storm cell to the surface than turbulent diffusion. Shearing of the pollutant cloud occurs in the upper level of the storm cell. Concentration reaching the anvil portion of the cloud is advected at a faster velocity than concentration in the lower levels of the storm; $5 \%$ of the concentration is advected out of the anvil into the stratosphere.

Results obtained with a modified Gaussian puff model were considered to be low and showed the inflexibility of the analytical solution to account for the transient nature of the vertical wind field. Ground-level $X / Q$ values were several orders of magnitude less than those obtained from the numerical method (within $20 \mathrm{~km}$ of the point of initial dispersion in the tornadic storm). Lateral spread of the concentration was significantly greater than that predicted by the three-dimensional numerical model. 


\section{REFERENCES}

1. T. T. Fujita. Review of Severe Weather Meteorology at Babcock and Wilcox Company, Leechburg, Pennsylvania. Department of Geophysical Sciences, University of Chicago, January 31, 1977, 24 p. (1977).

2. E. H. Markee and J. G. Berkerley. Technical Basis for Interim Regional Tornado Criteria. USAEC Report WASH-1300, U.S. Atomic Energy Commission, Washington, DC (1974).

3. K. C. Mehta, J. R. McDona1d, and D. A. Smith. Response of Structures to Extreme Wind Hazard at the Babcock and Wilcox Plant, Leechburg, Pennsylvania, Vol. I. Institute for Disaster Research, Texas Tech. University, Lubbock, TX 30 pp. (1977).

4. J. Mishima, L. C. Schwendiman, and J. A. Ayer. Airborne Release of Plutonium from $B$ \& $W$ Plant as a Result of Severe Wind Hazard. Battelle Pacific Northwest Laboratories, Richland, WA. (1978).

5. T. T. Fujita. Tornado Structure for Engineering Application with Design Basis Tornado Model (DBT-77) Department of Geophysical Sciences, University of Chicago, $111 \mathrm{pp}$. (1977).

6. T. T. Fujita. Workbook of Tornadoes and High winds for Engineering Applications. SMRP Research Paper 165, Department of Geophysical Sciences, University of Chicago, Savannah River Laboratory, Aiken, SC (1975).

7. D. W. Pepper. Dispersion of Small Particles in a Tornado. USERDA Report DP-1387, E. I. du Pont de Nemours and Company, Savannah River Laboratory, Aiken, SC, (1975).

8. W. E. Davis. An Estimate of In-Cloud Scavenging of a Tracer Injected into Two Frontal Storms. BNWL Report 2000, Pt. 3, Batelle Pacific Northwest Laboratories, 160-165 (1978).

9. C. F. Hane. "Scavenging of Urban Pollutants by Thunderstorm Rainfall: Numerical Experimentation." J. Appl. Meteorl., 17, 699-710 (1978).

10. E. A. Jessup. "Interpretations of Chaff Trajectories Near a Severe Thunderstorm." Month1y Weather Review, 100, 653-661 (1972).

11. R. P. Davies-Jones. Tornado Wind Fields. Presented Workshop on Dispersion and Deposition of Entrained Materials after a Tornado Strike, ANL, June 17-19 (1975). 
12. J. C. Fankhauser. "Thunderstorm-Environment Interactions Determined from Aircraft and Radar Observations." Monthly Weather Review, 99 (3) (1971).

13. D. W. Burgess, L. R. Lemon, and R. A. Brown. "Tornado Characteristics Revealed by Doppler Radar." Geo. Res. Letters, 2, $183(1975)$.

14. J. R. Eagleman and W. C. Lin. "Severe Thunderstorm Internal Structure from Dual-Doppler Radar Measurements." J. Appl. Meteorol., 16, p. 1036 (1977).

15. R. A. Kropfli and L. J. Miller. "Thunderstorm Flow Patterns in Three Dimensions." Mo. Wea. Rev., 103, 70-71 (1975).

16. F. A. Brandes. "Flow in Severe Thunderstorms Observed by DualDoppler Radar." Mo. Wea. Rev., 105, 113-120 (1977).

17. F. M. Heymsfield. "Kinematic and Dynamic Aspects of the Harrah Tornadic Storm Analyzed from Dua1-Doppler Radar nata." Mo.

Wea. Rev., 106, 233-254 (1978).

18. S. L. Barnes. "Oklahoma Thunderstorms on 29-30 April 1970. Part I: Morphology of a Tornadic Storm." Mo. Wea. Rev., $106,685-696(1978)$.

19. P. S. Ray. "Vorticity and Divergence Fields within Tornadic storms from Dual-Doppler observations." J. Appl. Meteorol., 15 (8) (1976).

20. L. R. Hoxit, C. F. Chappell, and J. M. Fritsch. "Formation of Mesolows or Pressure Troughs in Advance of Cumulonimbus Clouds." Mo. Wea. Rev., 104, 1419-1428 (1976).

21. P. S. Ray, K. K. Wagner, K. W. Johnson, J. J. Stephens, W. C. Bumgarner, E. A. Mueller. "Triple-Doppler Observations of a Convective Storm." J. App1 Meteorol., 17, 1201-1212 (1978).

22. J. B. Klemp and R. B. Wilhelmson. "The simulation of ThreeDimensional Convective Storm nynamics." J. Atmos. Sci., 35, 1070-1096 (1978).

23. A. J. Thorpe and M. J. Miller. "Numerical Simulations Showing the Role of the Downdraught in Cumulonimbus Motion and Splitting." Quart. J. R. Met. Soc., 104, 873-893 (1978).

24. Y. J. Lin and P. T. Chang. "Some Effects of the Shearing and Veering Environmental Wind on the Internal Dynamics and Structure of a Rotating Supercel1. Thunderstorm." Mo. Wea. Rev., $105,987-997$ (1977). 
25. R. E. Schlesinger. "A Three-Dimensional Numerical Model of an Isolated Thunderstorm: Part I. Comparative Experiments for Variable Ambient Wind Shear." J. Atmos. Sci., 35, 690-713 (1978).

26. J. H. Golden and D. Purcell. "Airflow Characteristics Around the Union City Tornado." Mo. Wea. Rev., 106, 3-11, (1978).

27. R. P. Davies-Jones and E. Kessler. Tornadoes. Chapt. 16, Weather and Climate Modification. ed. W. M. Hess, New York, John Wiley and Sons.

28 R. Rotunno. "Numerical Simulation of a Laboratory Vortex." J. Atmos. Sci., 34, 1942-1956 (1977).

29. R. Orville, "Wind Profile in the Subcloud Layer of a Thunderstorm." EOS, Trans. Amer. Geophy. Union, 58, 610-611 (1977).

30. C. W. Newton. "Circulations in Large Sheared Cumulonimbus." Tellus, 18 (4) (1966).

31. D. W. Pepper and A. J. Baker. "A High-Order Accurate Numerical Algorithm for Three-Dimensional Transport Prediction." Computers and Fluids, $\underline{8}, 371-390$ (1980).

32. K. A. Browning and R. J. Donaldson, Jr. "Airflow and Structure of a Tornadic Storm," J. Atmos. Sci., 20, 533-545 (1963).

33. C. C. Shir and L. J. Shieh. "A Generalized Urban Air Pollution Model and Its Application to the Study of SO2 Distribution in the St. Louis Metropolitan Area." J. Appl. Meteorol., 13, 1.85-204 (1974).

34. K. W. Ragland and R. L. Dennis. "Point Source Atmospheric Diffusion Model with Variable Wind and Diffusivity Profiles." Atmos. Environ., 9, 175-189 (1974).

35. E. M. Agee. "Some Inferences of Eddy Viscosity Associated with Instabilities in the Atmosphere." J. Atmos. Sci., 32, 642-646, (1975).

36. A. S. Frisch and R. G. Strauch. "Doppler Radar Measurements of Turbulent Kinetic Energy Dissipation Rates in a North eastern Colorado Convective Storm." J. Appl. Meteorol., $15(1976)$.

37. P. H. Hildebrand. "A Radar Study of Turbelent Diffusion in the Lower Atmosphere." J. App1. Meteorol., 16, 493-510 (1977). 
38. H. H. Lettau. "Wind Profile, Surface Storms, and Geostrophic Drag Coefficients." Adv. in Geophysics, 6, Atmospheric Diffusion and Air Pollution, Academic Press, New York, 241-256 (1959).

39. K. L. Calder. "The Numerical Solution of Atmospheric Diffusion Equation by Finite Difference Methods." Dept. of Army Tech. Memo, 130 (1968).

40. S. D. Reynolds, P. M. Roth, and J. H. Seinfeld. "Mathematical Modeling of Photochemical Air Pollution: Formulation of the Model." Atmos. Environ., 7, 1033 (1973).

41. S. K. Kao. "A Model for Turbulent Diffusion Over Terrain." J. Atmos. Sci., 33, 157-158 (1976).

42. W. G. Slinn. In-Cloud Scavenging of Aerosol Particles Annual Report for 1974. Part III. Atmospheric Sciences. USAEC Report BNWL-1950 (Pt. 3), Battelle Northwest Laboratories, Richland, WA, February (1975).

43. W. G. N. Slinn. Washout Ratios for Particulate Pollution with an Application to Convective Storms. USAEC Report BNWL $2000 \mathrm{Pt}$. 3, Battelle Pacific Northwest Laboratories, 139-142 (1978).

44. A. N. Dingle. "Scavenging of Tracer in Severe Storms." Pub1 ished in Precipitation Scavenging, USAEC Tech. Report edited by R. J. Engelman and W. G. N. Slinn, Pp. 21-33 (1970).

45. R. C. Sklarew, A. J. Fabrick, and J. E. Prayer. "Mathematical Modeling of Photochemical Smog Using the PICK Method." J. Air Pollut. Control. Assoc., 22, pp. 865-869 (1972).

46. R. Lange. ADPIC: A Three Dimensional Computer Code for the Study of Pollutant Dispersal and Deposition Under Complex Conditions. UCRL-51462, Lawrence Livermore Laboratory, 60 pP. (1973).

47. B. A. Egan and J. R. Mahoney. "Numerical Modeling of Advection and Diffusion of Urban Area Source Pollutants." J. App1. Meteorol., 11, 312-322 (1972).

48. D. W. Pepper and P. E. Long. "A Comparison of Results Using Second Order Moments with and without Width Correction to Solve the Advection Equation." J. Appl. Meteorol., 17 pp. (1978).

49. P. E. Long and D. W. Pepper. "A Comparison of Six Numerical Schemes for Calculating the Advection of Atmospheric Pollution." Presented at the Third Symposium on Atmospheric Turbulence, Diffusion, and Air Quality, Raleigh, NC, October 19-22, 1976. 
50. L. P. Prahm and L. B. Pedersen. "Comments on Numerical Modeling of Advection and Diffusion of Urban Area Source Pollutants." J. Appl. Meteoro1., 17, 1243-1244 (1978).

51. L. B. Pedersen and L. P. Prahm. "A Method for Numerical Solution of the Advection Equation." Tellus, 26, 594-602 (1974).

52. T. T. Fujita, M. R. Hjelmfelt, and S. A. Changnon. 'Mesoanalys is of Record Chicago Rainstorms Using Radar, Satellite, and Rain Gauge Data." Tenth Conference on Severe Local Storms, October 18-21, Omaho, Nebraska, p. 65-72 (1977).

53. W. G. N. Slinn. In-Cloud Scavenging of Aerosol Particles. USAEC Report BNWL-1950, Pt. 3, Battelle Pacific Northwest Laboratories, 114-118 (1975).

54. I. I., Burtsev, L. V. Burtseva, and S. G. Malakov. "Washout Characteristics of a $32 p$ Aerosol Injected to a Cloud." Atmospheric Scavenging of Radioisotopes, B. Styra, et al., (eds.), Proc. Conf. in Palanga, June 7-9, 1966. Trans. as TT-69-5509 from NTIS, springfield, VA, (1970). 


\section{APPENDIX}

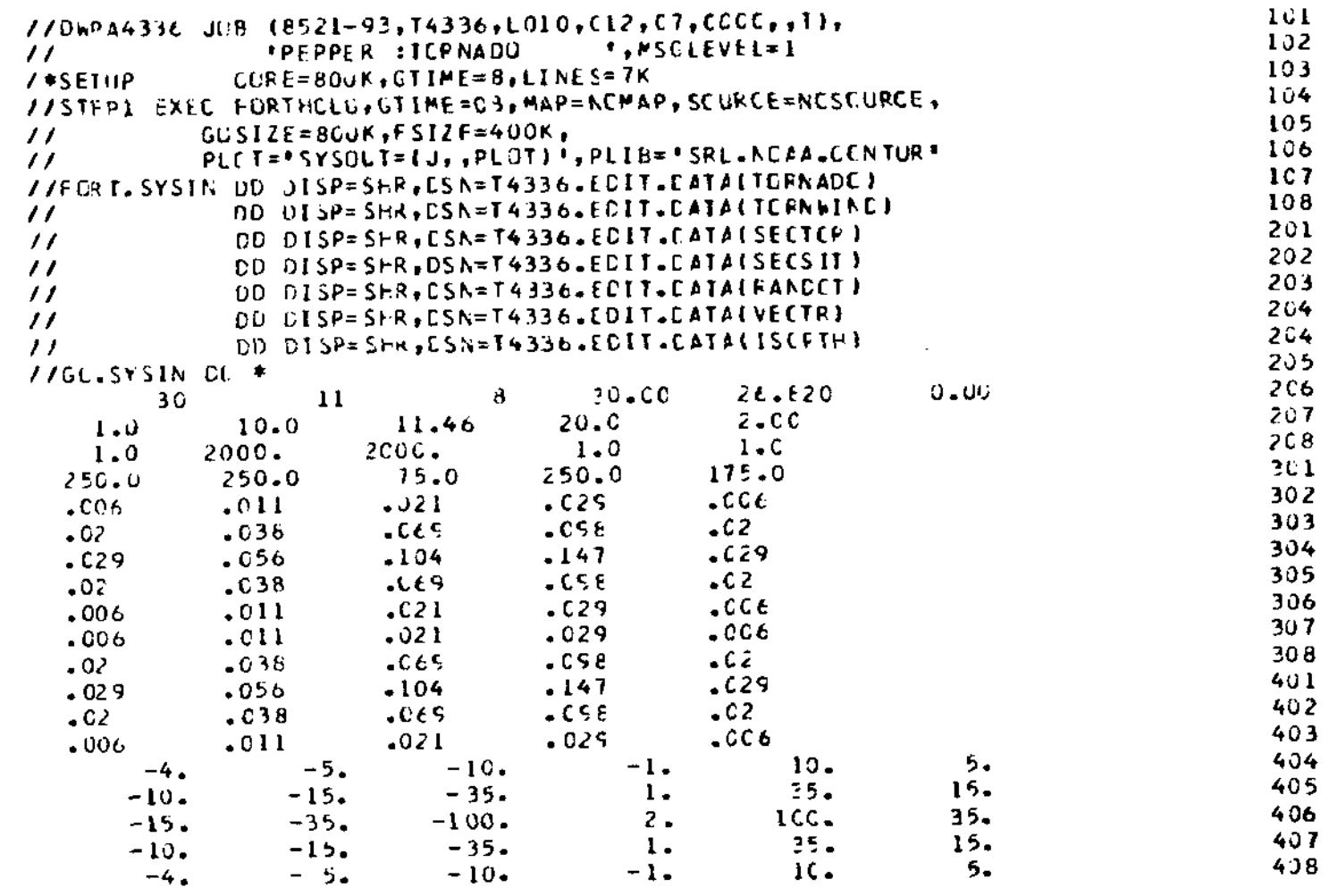




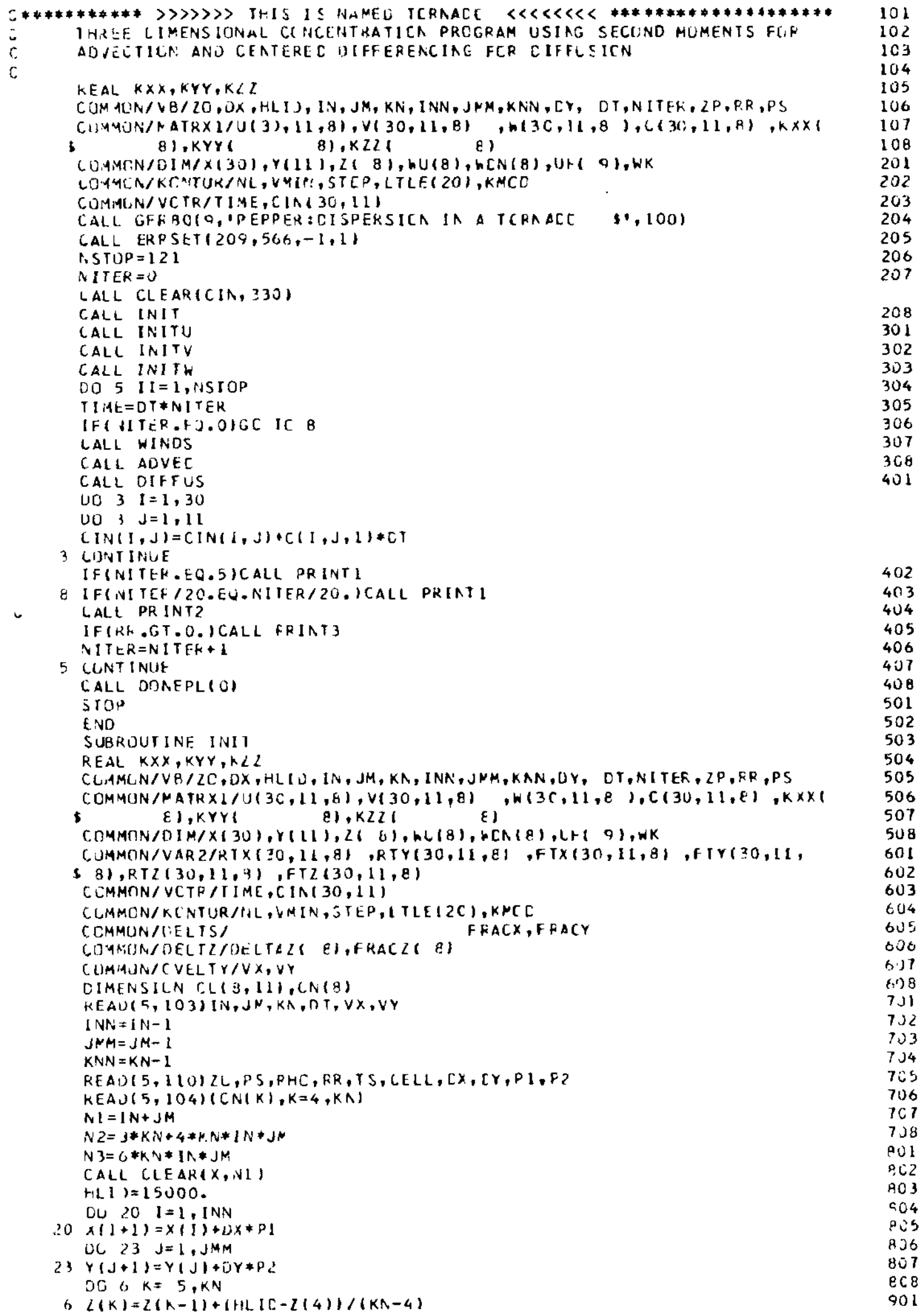


DO $4 K=1, K N N$

4 DELTAZ(K) $=Z(K+1)-Z(K)$

DELTAZ(KN) $=Z(K A)-Z(K$ KNA

PRINT $100, I N, D X, V X, V Y$

年,

PRINT 101,ZC,PS, RHE,RR,TS,CELL

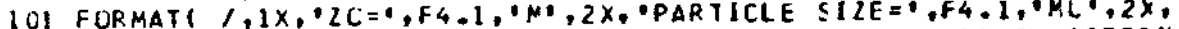

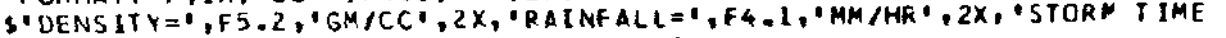

$3=1, F 4,1$, HR $, 2 X, 1, C$, CELLS $=1, F 3.0,1$,

CALL CLFAR (U,N2)

CALL CLEAR(RTX, $\cap 3)$

READ $(5,102)((C L(1, J), I=3,7), J=4,8)$

DO $10 \mathrm{~K}=4, \mathrm{KNN}$

DO $10 \quad J=4,8$

UO $10 \quad I=3,7$

$10 C(I, J, K)=C N(K) * C(I, J)$

REAU $(5,102)(\{(L L, 1, J), I=3,7\}, J=4,8)$

$K=K N$

DO $12 \mathrm{~J}=4,8$

UC $12 \quad I=3,7$

$12 C(I, J, K)=C N(K) * C L(I, J)$

$0011 K=4, K N$

DO $11 \mathrm{~J}=4,8$

DO $11 \quad I=3.7$

If $(C(I, J, K), E Q \cdot, \cdot 100$ TC 11

$R T X(I, J, K)=1: 0$

RiY $(I, J ; K)=1.0$

$K T Z(I, J, K)=1.0$

11 CCNTINUF

110 FORMAT (5F10.4)

102 FURMAT (5F10.4)

103 FORMAT $13110,3 \mathrm{~F} 1 \mathrm{C} .21$

104 FORMATISF10.0)

KETURN

END

SUBROUT INE PR INT

COMMON/VE/ ZO, OX, FL(1), IN,JM, KN, INN, JMM,KNN,OY, DT,NITER, ZP,RR,PS

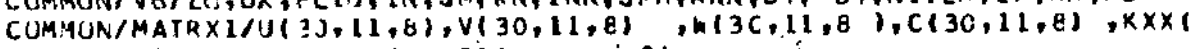
8), KYY Q), K22 (e)

DI MENSION OUMY(30,11), LS 30,11$)$, DUM $1(30,11)$, CUM $2(30,11)$

COMM(IN/DIM/X130), Y(11), 21 8), hU(8), hCN(8), UH( 91, WK

COMMUN/YCTR/TIHE, CIN $(30,11)$

COMMON/CVELTY/VX, VY

DUMN $=V X * .02$

DU $6 \quad I=1, I N$

DU $6, j=1, j 4$

DUMI(I, J)=U( $[\because, J, 4) \div .02$

I F SOUMI (I, J).EQ.OUMM) DUMI $(1, d)=0$.

$6 \operatorname{DUM} 2(I, J)=V(I, J, 4) * .02$

CALL VECTRIOUMI, CUML, IN, JN, CLPYI

SUM $=0.0$

DC: $B \quad K=1, K N$

DO $8 \quad l=1, I N$

DO \& $\mathrm{J}=1, \mathrm{JM}$

a $S U H=S{ }^{\prime}+C([, J, K)$

$K=1$

$F L A G=0$.

DO $9 \quad 1=1, I N$

UC $9 \quad J=1, J M$

$J J=J \cdot Y-J+1$

OUMY $(I, J)]=C(1, J, K) /(0 X * 0 Y * Z 12) * 1 C(C$,

If ( DUMY $(I, J J), G T \cdot 0$.$) IF LAG =1$.

$(S(1, J)=C(1, J, K)$

9 CUNTINUE

IF IFLAG.EQQ0.1GC IC I

PRINT ?

2 HURMATIIHI, 50X, 'AIR CINCENTRATION', I

WFITF(t, IUI)

WRITE $(5,102) K$, I INE, SUM

IR $R$ TTE $(6,107\}\{1,1=1,30\}$

[U $5 \mathrm{~J}=1, \mathrm{JH}$

$J J=J M-J+1$

1102

1103

1104

1105

1106

1107

$110 \mathrm{~B}$

1201

1202

$12 \mathrm{C3}$

1204

1205

1206

1207

1208

1301

1302

1303

1304

1305

1306

1307

1308

1401

1402

1403

1404

1405

1406

1407

1.408

1501

1502

I503

1504

1505

1506

1507

1508

1601

1602

1603

1604

1605

1606

1607

1608

1701

1702

1703

1704

1705

1706

1707

1708

1801 
FORMATIIHL,5OX WRIIE $(6,101)$

WRITE $(6,102) K, T I N E$, SUM

DO $15 I=1, I N$

$I I=I N-I+I$

15 WRITE $(6,104)$ (CINIII,J),J=1,111

CALL ISDLOGIDUMY, IN, JM, DUMI,CLMZI

UU $3 \quad I=1, I N$

DO $3 \quad J=1, j M$

$3 \operatorname{DUMY}(I, J)=C(I, J, 1) /(D X * D Y * Z(2) * 10 \mathrm{CO}$,

IFINITER.EQ.120ILALL SECTOR (DUMY, IN, JM)

IFINITER.EQ.120ICALL SECSIT (CLMY,IA,JM,VX)

1 CONTINUE

CALL PRINT2

101 FCRMATE $1, T 46,1 X-Y$ PLAME ',

102 F CRMAT $(20 x, ' K=1,15,10 x$, IIME= , f $10, z, 10 x$, 'SLM $1,1 P E 10,3)$

103 F GRMAT $(30(\{4))$

104 FURMATC IX, IP ILE II.3)

107 FORMATI $1,1 \times, 30(13,(x), 1)$

RETURN

END

SUBROUT INE PRIINTZ

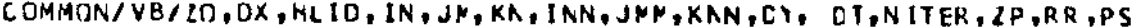

1704

1705

1706

1708

1801

1802

1803

$1 \mathrm{RO}$

1805

1806

1807

1808

1901

1902

1903

1904

1905

1905

1906

1907

1908

2001

COMMON/MATRXI/U( $30,11, B), V(30,11,8), \ln (3 \mathrm{C}, 11,8), \mathrm{C}(30,11,8), \mathrm{K} \times \mathrm{C}$ E) 8I, KYYI B), $K 221$

2002

2003

2004

$2 \mathrm{CCS}$

COMMON/ VCTR/T IME CIN( 30,11

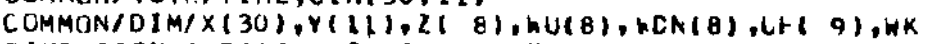

DIMENSION ZPT $(3 \mathrm{C}), \operatorname{LS}(30,8), \operatorname{CUMY}(30,8), \mathrm{W} 1(3 \mathrm{C}, 8)$, CUM $1(30,8), U N(30, E)$

$J=6$

$N 1=I N * K N$

UO $4 \quad I=1, I N$

$004 \quad K=1, K N$

$H I(I, K)=H([, J, K) * ., C 1$

$U N(I, K)=U(I, d, K) * 01$

UUMY $(I, K)=C(I, \ldots, k)$

$4 L S(I, K)=(I I, J, K)+S I \operatorname{GN}(0.5$,

CALL VECTRIUN,W $1, I A, K A, D U M L)$

$C(1, j, x)$

2006

$2 \mathrm{CO} 7$

2008

2101

2.102

2103

2104

2105

2106

2107

2108

2201

2202

DC $7 \mathrm{~K}=1,3$

DC $7 \quad I=2, I N$

$\mathrm{FLAG}=0$.

I F(W) $(1, K), L T,-, C C 5) F L A G=1$

IFIFLAG .EO.1. ICALL RANDOT (FLCATII),FLCATIN,IA, KN, IY)

2203

2204

2205

2206

2207

2208

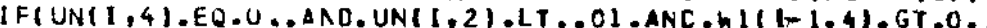

SCALL RANDCT(FLJAT(I),FLOAT(K),IA,KA, IY)

$I Y=I Y+2$

7 CONTINUE

CALL RECON

CALL I SOPTH(DUMY, IN,KN,OUML, $, \ldots, 0.5)$

DO $40 \quad I=1, I N$

ZPT $(I)=$ DUAY $(1, I H(C X * D Y * Z(2) * 1000)$.

40 IF(ZPTII).LE. O. IZPT $(I)=1.00 E-20$

CALL YLGPLT $(X, \angle P T, T O)$

101 FCRMATI/1,46X," $X-2$ PLANE IN J DIRECTICN", I

102 FORMAT( $30 X$, "TIME $=$, F 10.2$)$

103 FORMAT $(30114))$

ICT FORMATI $1,1 \times, 30(13,1 \times 1,1)$

RETUP.N

END

SUBROUTINE PKLNT3

COMMUN/VE/ZO,DX,HLI I, IN, JM, KN, INN, JNM,KAN,DV, CT,NITER, ZP,RR,PS

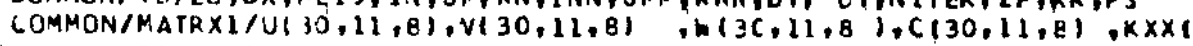

8),KYYI $\quad$ 8), KZZ

DIMENSION DUMY $(30,11), L S(30,11), \times \times(30,11)$, CUM $1(30,11), D(N 213 C, 11)$ COMMCN/CVELTY/VX, VY

COMMON/VCTR/TIME, CIN 30,11$)$

N $2=I N * J M$

IF (N) TER.E).JICALL CLEAR(XX,A2)

2308

2401

2402

2403

2404

2405

2406

2407

2408

2501 


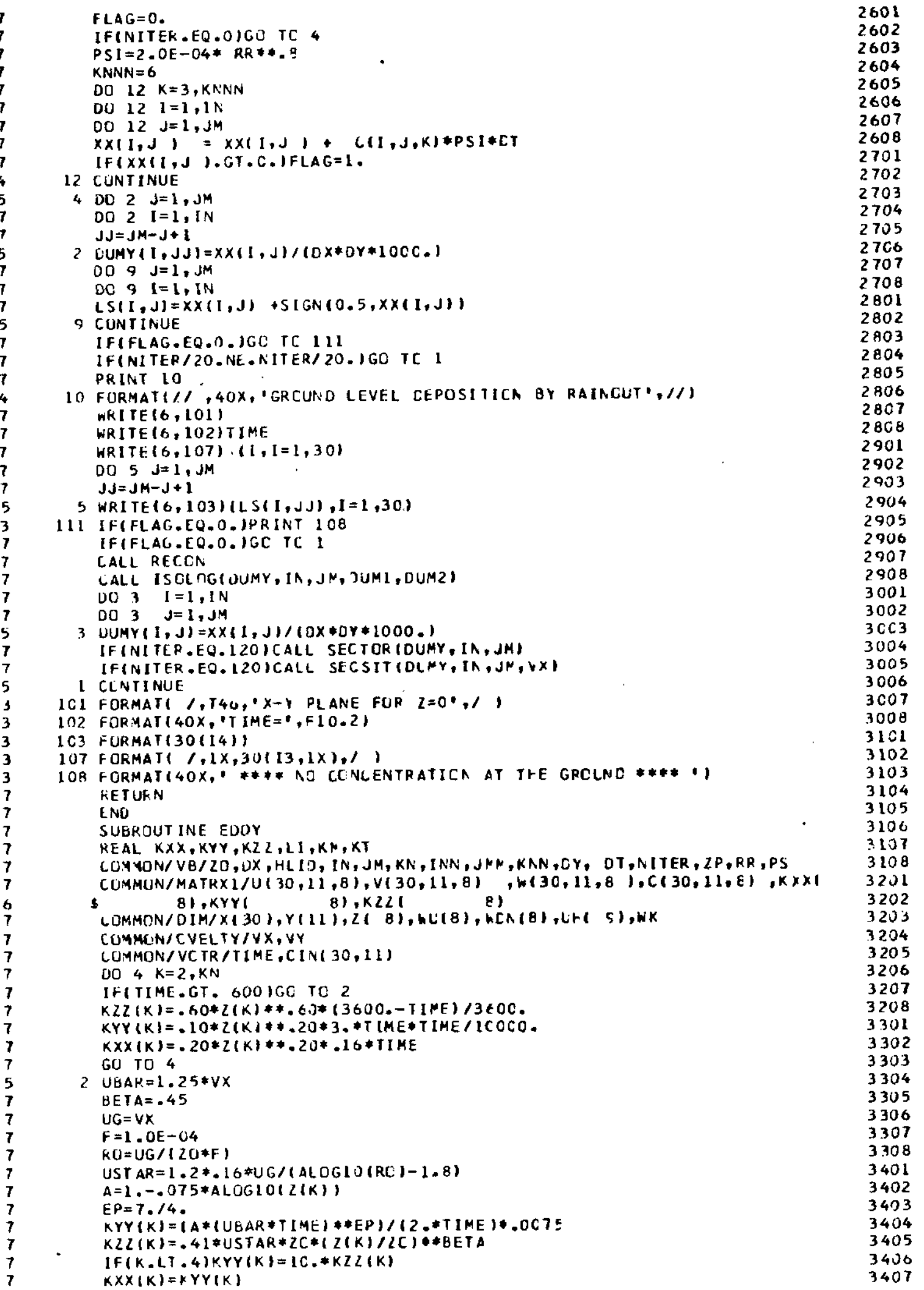




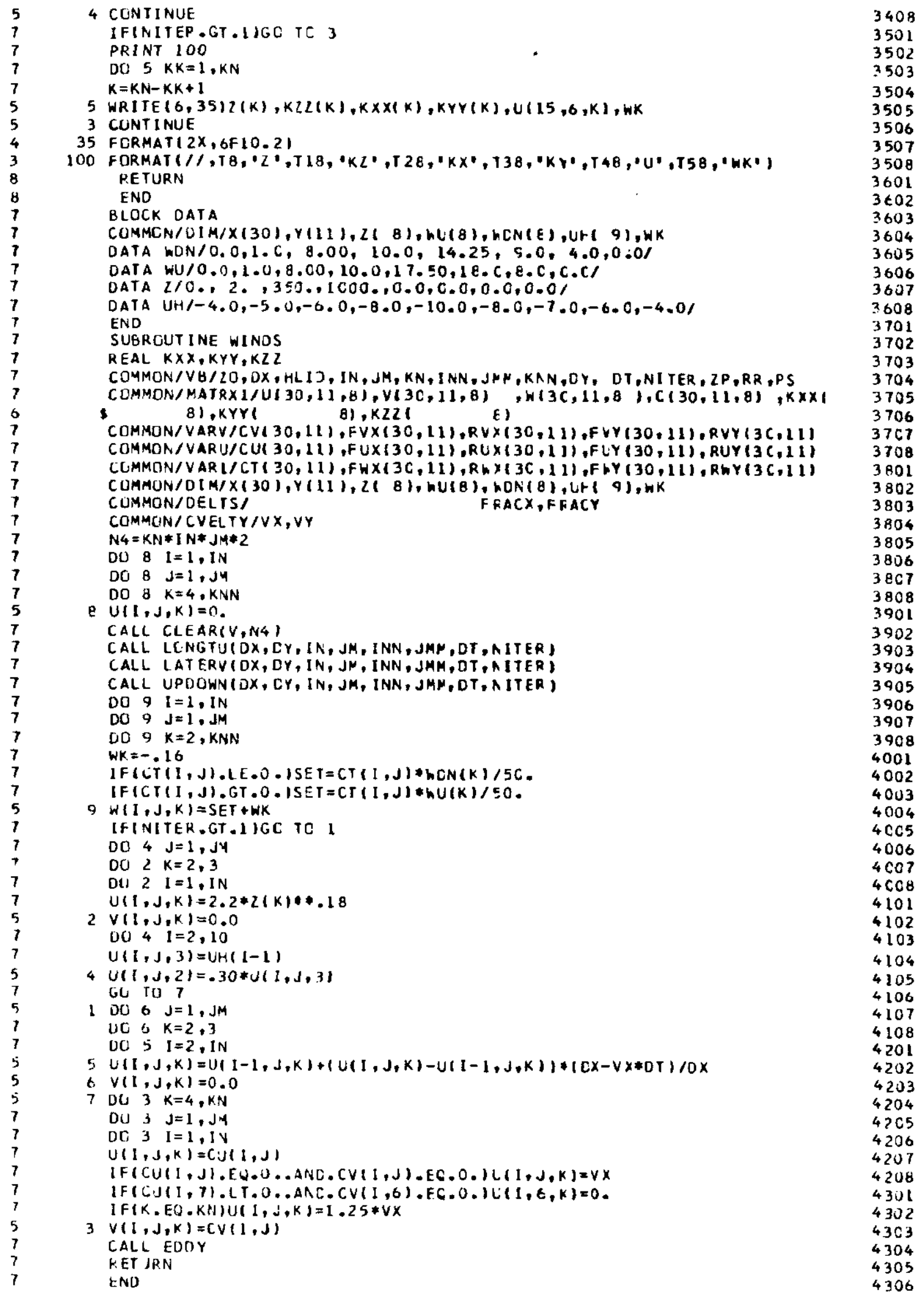


SUBRUUTINE DIFFLS

REAL KZZ,KLZK,KZZP,KZZM,KXX,KYY,KXXK,KYYK

COMMUNIVB ZR, UX, HLID, IN, JM, KA, INN, JYM, KNN,DY, DT,NITER, ZP,RR,PS

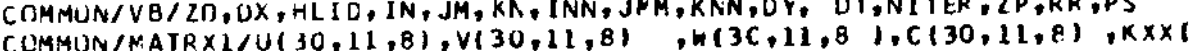
Q) , KYYI 8), $K 221$ E)

4402

4403

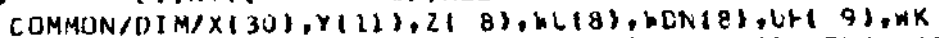

COMMUN/CALGOR/O (30,Il,8),FXA $(30,11, E)$,FYA $(30,11,8)$,FZA

4404

$1(30,11,8), R \times A(30,11,8)$, RYA $(30,11, E), R Z A(30,11,8)$

CLMMON/DELTS/

FAACX, FAACY

COMMON/DELTZ/OELTAZ\& \&),FRACZ( E)

COYMUN/VAR2/RTX $(30,11,8), R T Y(30,11,8), F T X(30,11,8)$,FTY(30,11.

S $B), R T 2(30,11,8), F(2(30,11,8)$

CALL SLAVEG

DU $10 \quad K=1, K N$

DO $10 \quad \mathrm{~J}=1, \mathrm{JM}$

$0010 \quad I=1, I N$

$U(I, J, K)=C(I, J, K)$

$R X A(I, J, K)=R I X(I, J, K)$

$R Y A(I, J, K)=R T Y(I, J, K)$

$R Z A(I, J, K)=R T Z(I, J, K)$

FXA $(I, J, K)=F T X(1, J, K)$

FYA $(1, J, K)=F T Y(I, J, K)$

FZAII,J,K)=FTZ(I,J,K)

20 CONT INUE

$R B=1.0$

$R T=0.95$

$006 \quad I=2, I N N$

$006 \quad j=2, J M M$

$006 \quad K=1, K N N$

$K X X K=K X X(\quad K)$

$K Y Y K=K Y Y(\quad K)$

$A X M=K X X K * D T /(D X * D X)$

$A X P=A X M$

$A Y M=K Y Y K * D T /(D Y * D Y)$

$A Y P=A Y M$

IF (K.EQ.1)GO TO A

IF(K.EO.KNN)GO TO $S$

$D Z P=Z(K+2)-Z(K)$

$D Z=2(K+1)-Z(K-1)$

$K Z Z P=K Z Z(K+1)$

$K L L K=K L L(K)$

$K Z Z M=K Z Z(K-1)$

$A K M=A K P$

$\triangle K P=(K Z Z K+K Z Z P) * Z T /(O Z * C Z P)$

$D I F=1 .-A X P-A X M-A Y P-A Y M-\triangle X P-A K F$

IFI DIF.LT.D.JPR INT IOC

$C(I, J, K)=C(I, J, K) \neq C$ If $+A \times P * 0(I+1, J, K)+A \times M *$

$\$ 0(1-1, J, K)+A Y P * C(1, J+1, K)+A Y M * C(I, J-1, K)+A K P * C(I, J, K+1)+A K M *$

$\$ 0(1, J, K-1)$

IF(CII,J,K).LT. I.E-LO)GO TO 30

$F T X(I, J, K)=(J(I, J, K) * F X A(I, J, K) * D(F+A X P * C(I+1, J, K) * F X A(I+1, J, K)$

$S+A X M * D(1-1, J, K)+F X A(I-1, J, K)+A Y P * C(1, J+1, K) * F X A(1, J+1, K)+A Y M *$

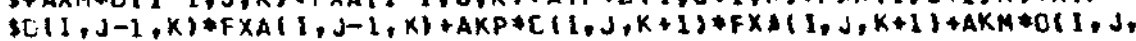
SK-1)*FXA(I,J,K- l))/C(I,J,K)

$F T Y(I, J, K)=(C 1 I, J, K) * F Y A(I, J, K) * U(F+A X P * C(I+1, J, K) * F Y A(I+1, J, K)$

$S+A X M * O(I-1, J, K) * F Y A(I-1, J, K)+A Y P * C(1, J+1, K) * F Y A(I, J+1, K)+A Y A *$

SU $(1, J-1, K) * F Y A I 1, j-l, K)+A K P *[(1, J, K+1\} * F Y A(1, \ldots, K+1)+A K K *(1, J$,

$\$ K-1)$ FYYA $1, J, K-1) 1, C(I, J, K)$

FI $Z(1, J, K)=(U(I, J, K) * F Z A(I, J, K) * 0 I F+\Delta X P * C(I+1, J, K) * F Z A(I+1, J, K)$

$S+A X M * O(I-1, J, K) \oplus F Z A(I-1, J, K)+A Y P * C(I, J+1, K) * F Z A(I, J+1, K)+A Y M *$

$\$ 0(1, J-1, K) * F(A \mid 1, J-1, K)+A K P+[(1, J, K+1) * F 2 A(1, J, K+1)+A K M * C(1, J$, SK-1)*FZA $(I, J, K-1), C(I, J, K)$

GO TO 6

B $\quad D Z P=2(2)-2(1)$

$\mathrm{DZ}=2(3)-Z(1)$

$K Z Z P=K Z Z(2)$

$K Z Z K=K Z Z Z 1)$

$A K P=(K \angle Z K+K Z \angle P) * J T /(0 Z * 0 Z P)$

CIF $=1 .-A X P *(2,-R E)-A X P-A X M-A Y P-A Y M$

IF(I)IF.LI.0.)PRI.VT 101

$C(I, J, K)=(I(1, J, K) * D[f+A \times P * O(1+1, J, K)$

4406

4407

4408

4501

4502

4503

4504

4505

4506

4507

4,508

4601

4602

4603

4604

4605

4606

4607

4608

4701

4702

4703

4704

4705

4706

4707

4708

4801

4802

4803

4804

4805

4806

4807

4808

4901

4902

4903

4904

4905

4906

4907

4908

5001

5002

5003

5004

5005

5006

5007

5008

5101

5102

5103

5104

5105

5106

5107

5108

5201

5202

5203

5204

5205 
$5+A X M * D(I-1, J, K)+A Y P * C(I, J+1, K)+A Y K * C(1, J-1, K)+A K P * 0(I, J, K+1)$

IF $(C I I, J, K), L T, I, E-10) G 0$ JO 30

$F T X(I, J, K)=(U(I, J, K) * F X A(I, J, K) * 0 I F+A X P \neq 0(I+1, J, K) * F \times A(I+1, J, K)$

$5+A X M * C(I-1, J, K) * F X A(I-1, J, K)+A Y P * C(I, J+1, K) * F X A(1, J+1, K)+A Y M *$

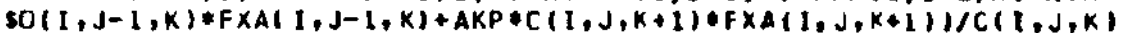

$F T Y(I, J, K)=(U(I, J, K) \neq F Y A(I, J, K) \neq D I F+A X P \neq 0(1+1, J, K) \neq F Y A(I+L, J, K)$

$S+A X M * O(I-1, J, K) * F Y A(I-1, J, K)+A Y P * C(I, J+1, K) \notin F Y A(I, J+1, K)+A Y * *$

$S U(I, J-l, K) * F Y A(I, J-l, K)+A K P * C(I, J, K+1 J * F Y A(I, J, K+1), / C(I, J, K)$

$F T Z(I, J, K)=(U(I, J, K) * F Z A(I, J, K) * 0 I F+A X P \bullet C I I+1, J, K I * F Z A(I+1, J, K)$

$\$+A X M * 0(I-1, J, K)+F Z A(I-l, J, K)+A Y P * C I I, J+1, K) * F Z A(I, J+1, K)+A Y M *$

$S O(I, J-1, K) * F L A I I, J-I, K)+A K P *(I, J, K+1) * F Z A(J, J, K+1), / C(1, J, K)$ GO 106

$9 \quad A K M=A K P$

OIF $=1 .-A K M *(2 .-R T)-A X P-A X M-A Y F-A Y M$

IFIOIF.LT.O.JPKIVT 102

$C(I, J, K)=O(I, J, K) \oplus J I F+A X P \oplus O(I+1, J, K)$

$S+A X Y * B(I-1, J, K)+A Y P \leqslant O(I, J+1, K)+A Y *(I, J-1, K)+A K M * J(I, J, K-1)$

IF $(C(I, J, K), L T, 1, E-10) G C$ JO 30

$F T \times(l, J, K)=(U(1, J, K) \neq F \times A(1, J, K) \neq 0 I F+A \times F * 0(1+1, J, K)+F \times A(1+1, J, K)$

$\$+A X M * O(I-1, J, K) * F X A(I-1, J, K)+A Y P * C(I, J+1, K) * F X A(I, J+I, K)+A Y N *$

$S O(I, J-1, K) * F X A(I, J-1, K)+A K M *[(I, J, K-1) * F X A(I, J, K-1)(C, I, J, K)$

$F T Y(I, J, K)=(D(I, J, K) * F Y A(I, J, K) * 0 I F+A X P * 0(I+1, J, K) * F Y A(I+1, J, K)$

$S+A X Y+O(I-1, J, K) \neq F Y A(I-1, J, K)+A Y P+(I, J+1, K)+F Y A(I, J+1, K)+A Y K$

$\$ O(1, J-1, K) * F Y A(I, J-1, K)+A K M * O(1, J, K-1) \oplus F Y A(I, J, K-1) / C(I, J, K)$

$F T Z(I, J, K)=(D(I, j, K) * F Z A(I, J, K) * 0 I F+A X P * C(I * 1, J, K) * F Z A(1 * 1, J, K)$

$S+A X M * C(I-1, J, K) * F Z A(I-1, J, K)+A Y P * C(I, J+1, K) * F Z A(I, J+1, K)+A Y * *$

$S[(J, J-1, K) * F Z A\} J, J-1, K)+A K M * C(I, J, K-1) \bullet F Z A(I, J, K-1),(C(I, J, K)$ GO TO 6

$30 F T X(I, J, K)=0.0$

FTY $(1, J, K)=0.0$

FT $Z(I, J, K)=0.0$

$C(I, J, K)=0.0$

6 CONTINUE

DO $39 I=2$, INN

$0039 \mathrm{~J}=2, \mathrm{JMM}$

DO $39 \mathrm{~K}=1, \mathrm{KNN}$

IF $(C(I, J, K) \cdot L T \cdot 1 . E-10100$ TO 50

KXXK=KXXY K)

$K Y Y K=K Y Y I \quad K)$

$A X: A=K X X K * 0 T /(D X * D X)$

$A X P=A X M$

$A Y M=K Y Y K * D T /(D Y * D Y)$

$A Y P=A Y M$

GO TO 40

$50 R T \times(1, J, K)=0.0$

$R T Y(I, J, K)=0.0$

$R T L(I, J, K)=0.0$

GO TO 39

$40 R \times 1=R \times A([, J, K)$

$R \times 2=R \times A(I+1, J, K)$

$R \times 3=R \times A(I-1, J, K)$

$R \times 4=R \times A(I, J+1, K)$

$R \times 5=R \times A(I, J-1, K)$

$S \times 1=R \times 1 * R \times 1+12, *(F \times A(J, J, K)-F T \times(I, J, K)) * 2$

$S \times 2=R \times 2 * R \times 2+12$. $*(F \times A I I+1, J, K)-F(X \times\{I+1, J, K\}) * 2$

$S \times 3=R \times 3 * R \times 3+12, *(F \times A(1-1, J, K)-F I \times(1-1, J, K)\} * 2$

$S \times 4=R \times 4 * R \times 4+12, *(F \times A \mid I, J+1, K)-F T \times(I, J * 1, K)) * 2$

$S \times 5=R \times 5 * R \times 5 * 12$ * $*(F \times A(I, J-l, K)-F(X X(I, J-1, K)) * 2$

$R Y I=R Y A(I, J, K)$

$R Y Z=R Y A(I+1, J, K)$

$R Y 3=R Y A(1-1, d, K)$

$R Y 4=R Y A(1, J+1, K)$

$R Y 5=R Y A(I, J-1, K)$

$S Y I=R Y I * R Y I+12$ * $(F Y A(I, J, X)-F I Y(I, j, X)) * * 2$

$S Y 2=R Y 2 * R Y 2+12 *(F Y A(I+1, J, K)-F T Y(I+1, J, K) * * 2$

$S Y 3=R Y 3 * R Y 3+12$. * (FYAI $I-1, J, K)-F T Y(I-1, J, K)) * 2$

$5 Y 4=K Y 4 * R Y 4+12$. $*(F Y A(I, J+1, K)-F T Y(I, J+1, K)) * 2$

$S Y 5=R Y S * R Y S+12$. * $(F Y A(I, J-1, K)-F P Y(I, J-1, K)) * 2$

$R Z I=R Z A(I, J, K)$

$R Z Z=R Z A(I+1, J, K)$

$R Z 3=R Z A(I-1, J, K)$

5301

5302

5303

5304

5305

5306

5307

5308

5401

5402

5403

5404

5405

5406

5407

5408

5501

5502

5503

5504

5505

5506

5507

5508

5601

5602

5603

5604

5605

5606

5607

5608

5701

5702

5703

5704

5705

5706

5707

5708

5801

5802

5303

5804

5805

5806

$5 \varepsilon 07$

5808

5901

5902

5903

5904

5905

5906

5907

5908

6001

6002

6003

6004

6005

6006

6007

6008

6101

6102

6103

6104 
$R Z 4=R Z A(1, J+1, K)$

6105

$R Z 5=R Z A(L, J-1, K)$

$S Z I=R Z I * R Z I+12$. $(F Z A(I, J, K)-F T Z(I, J, K)) * * 2$

$S Z Z=R Z 2 * R Z 2+12$. *(FLA(I+1,J,K)-FIZ(I*I,J,K))**2

$S Z 3=R Z 3 * R Z 3+12 *(F Z A(I-1, J, K)-F T Z(I-1, J, K)) * 2$

$S Z 4=R Z 4 * R Z 4+12$ * $(F Z A(J, J+1, K)-F T Z(I, J+1, K)) * 2$

$S Z 5=R Z 5 * R Z 5+12$ * $(F Z A(I, J-1, K)-F T 2(I, J-1, K) * 2$

IFIK.EO.I)GO TO 41

I $F(K . E Q . K N N) G U$ TC 42

$. U Z P=Z(K+2)-Z(K)$

$0 Z=2(K+1)-2(K-1)$

$K Z Z P=K Z Z 1 \quad K+11$

$K Z Z K=K Z Z I \quad K J$

$K Z Z H=K Z Z 1 \quad K-11$

$A K M=A K P$

$A K P=(K Z Z P+K Z Z K) * C I / 10 Z * D Z P)$

$R \times 6=R \times A(I, J, K+1\}$

$R \times 7=R \times A(1, J, K-1)$

$S \times 6=R \times 6 * R \times 6+12$. $(F \times A(I, J, K+1)-F T \times(1, J, K+1) * 2$

$S \times 7=R \times 7 * R \times 7+12 * *(F \times A(I, J, K-1)-F T \times(1, J, K-1)) * 2$

$R Y G=R Y A(I, J, K+1)$

$R Y 7=R Y A(I, J, K-1)$

SY $6=R$ Y $6 * R$ R $6+12$. * (FYA $(I, J, K+1)-F$ JY $(1, J, K+1) * 2$

SY $7=R Y 7 * R Y 7+12$ * (FYAI I, J,K-1)-FTY I , J,K-1)\}*2

$R Z 6=R Z A(I, J, K+1)$

$R Z 7=R Z A(I, J, K-1)$

$S \angle 6=R 26 * R Z 6+12 *(F Z A(I, J, K+1)-F T Z(I, J, K+1)) * 2$

$S Z 7=R Z 7 * R Z 7+12$. $*$ IFLAPI,J,K-1)-FTZ(I,J,K-1) $) * 2$

UIF $=1 .-A X P-A X M-\triangle Y P-A Y M-A K P-A K Y$

$R T \times(I, J, K) \neq(U(I, J, K) * 5 \times 1 * 0 I F+C(I * 1, d, K) * A \times P * S) 2+O(I-1, J, K) * A \times M$ $S * 5 \times 3+C(1, J+1, K) * A Y P * S \times 4+0(1, J-1, K) * A Y \mu * S \times 5+C(1, J, K+1) * A K P * S \times 6+$ $S C(I, J, K-1) * A K M * S X 7) / C(I, J, K)$

KTY $(I, J, K)=(0(I, J, K) * 5 \times 1 * 0 I F+0(I * 1, j, K) * A \times P * S Y 2 * U(I-1, J, K) * A \times M$ $\$ * S Y 3+0(I, J+1, K) * A Y P * S Y 4+0(I, J-1, K) * A Y K * S Y \leq * C(I, J, K+1\} * A K P * S Y \in+$ $\$ O(I, J, K-1) * A K M * S Y 7\} / C(1, J, K)$

$R T Z(I, J, K)=(O(I, \sim, K) * S Z 1 * 0 I F+C(I+1, \ldots, K) * A \times P * S Z 2 * 0(I-1, J, K) * \Delta \times M$ $\$ * S 23+0\{1, j+1, K) * A Y P * S 24+0(1, j-1, K) * A Y M * S 2 \leq+C(1, J, K+1) * A K P * 52 \in *$ LQ $(I, J, K-1) * A K M * S L 7) /(,(I, J, K)$ Gu 1043

$41 \quad D Z P=2(2)-2(1)$

$\mathrm{D} Z=2(3)-2(1)$

$K Z Z P=K Z Z(2)$

$K Z Z K=K Z Z(1)$

$A K P=(K Z Z P+K Z Z K) * 0 T /(D Z * D Z P)$

$D I F=1 .-A X P *(2 .-P B)-A X P-A X Y-A Y P-A Y N$.

$P \times 6=R \times A(I, J, K+1)$

$S \times 6=R \times 6 * R \times 6+12 *(F \times A(I, J, K+1)-F\} \times(1, J, K+1)) * 2$

$R Y G=R Y A(1, J, K+1\}$

$S Y G=2$ Y $6 * R Y G+12$. (FYAI 1,J, K+1)-FTY(I, J,K+1) $* 2$

$K \angle 6=R Z A(1, J, K+1)$

$S Z 6=R Z 6 * R Z 6+12 *(F Z A(I, J, K+1)-F T Z(1, J, K+1)) * 2$

$R T X(I, J, K)=(011, J, K) * S \times 1 * D I F+0(1+1,1, K) * A \times P * S \times 2+0(1-1, J, K) * A \times M$

$\$ * S \times 3+O(I, J+1, K) * A Y P * S \times 4+0(1, J-1, K) * A Y * * S \times 5+[(I, J, K+1) * A K P * 5 \times()$

$s / C(I, J, K)$

$R \operatorname{TY}(I, J, K)=(O(I, J, K) * S Y I * 0 I F+C(I+1, \ldots, K) * A \times F * S Y 2+O(I-1, J, K \mid * A \times M$

\$*SY $3+[)(I, J+1, K) * A Y \rho * S Y 4+D(I, J-1, K) * A Y K * S Y 5 * C(I, J, K+1) * A K P * S Y G)$

$4(C, J, K)$

$K J Z(I, J, K)=(O(I, J, K) * S L I * O I F+O(I+1, J, K) * A \times P * S Z 2+O(I-1, J, K) * A \times \psi$

$s * S Z 3+U(1, J+1, K) * A Y P * S 24+0(I, J-1, K) * A Y M * S Z E * C(I, J, K+1) * A K P * S Z \in)$

$\$ / C(I, J, K)$

GO 1043

42 AKM $=A K P$

UIF $=1 .-A K M *(2 .-R I)-A X P-A X M-A Y P-A Y N$

$K \times T=R \times A(I, J, K-1)$

$S \times 7=R \times 7 * R \times 7+12$. $*(F \times A(I, J, K-1)-F T \times(I, J, K-1) * 2$

$K Y T=R Y A(I, J, K-1)$

$S Y 7=R Y 7 * R Y 7+12, *(F Y A(1, J, K-1)-F T Y(1, J, K=1) * * 2$

RZT =RZACI, J,K-1)

$S \angle 7=R Z 7 * R Z 7+12 *(F Z A(1, J, K-1)-F T Z(I, J, K-1) * 2$

$R T \times(I, J, K)=(0(1, J, K) * S \times 1 * 0 I f+0(I+1, J, K) * A \times P \neq S \times 2+0(I-1, J, K) * A, K$

$5 * 5 X j+0(I, J * 1, K) * 1 Y P * 5 X 4+D(I, J-1, K) * A Y * * S X E+[(I, J, K-1) * A K M * S \times])$

6108

6201

6202

6203

6204

6205

6206

6207

6208

6301

6302

6303

6304

6305

6306

6307

6308

6401

6402

6403

6404

6405

6406

6407

6408

6501

6502

6503

6504

6505

6536

6507

6508

6601

6602

6603

6604

6605

6606

6607

6608

6701

6702

6703

6704

6705

6706

6707

6708

6801

6802

6803

6904

6845

6806

6807

$68 \mathrm{CB}$

6901

6902

6903

6904

6905

6906

6907

$69 \mathrm{CB}$

7001

7002

$7 \mathrm{CO} 3$ 
$S /(I, J, K)$

$R T Y(I, J, K)=(O(I, J, K) * S Y I * D I F+C(I+1, J, K) * A \times P * S Y 2+D(I-1, J, K) * A \times M$

S*SY $3+O(1, J+1, K) * A Y P * S Y 4+O(I, J-1, K) * A Y N * S Y 5+[(I, J, K-1) * A K * S Y]$

7005

$S / C(I, J, K)$

$R T L(I, J, K)=(0(I, J, K) * S 2 I * 0 I f+C(I+1, j, K) * A \times P * S Z 2+0(I-1, J, K) * A \times M$

7006

$\$ * S Z 3+0(1, J+1, K) * A Y P * S 24+0(I, J-1, K) * A Y K * S Z 5+C(1, J, K-1) * A K * S 27)$ $s /(1, J, k)$

$43 R T Z(I, J, K)=S Q R T(R T Z\{I, J, K))$

$R T X(I, J, K)=S O R T(R T X(I, J, K))$

$R T Y(I, J, K)=S O R T(R T Y(I, J, K))$

39 CONTINUE

100 FORMAT $110 \times$, DIF FOR $0<Z<K N$ IS $\left.<0^{\circ}\right)$

101 FORMATI $10 X, \circ D I F$ FCR $Z=1$ I $S<0 \% 1$

102 FORMAI ( $10 X$, DIF FCR $Z=K N$ IS $<0$. RETURN

END

SUBROUT INE ADVEC

CONMON/VB/ZO,DX,KLID,IA, JP, KN, INN, JPN, KNN,CY, DT,NITER, ZP,RR,PS

7008

7101

7102

7103

7104

7105

7106

7107

7108

7201

7202

7203

7204

7205

COMMON/MATRX1/U $(30,11,8), V(30,11,8), \operatorname{mi}(30,11,8,, \mathrm{C}(30,11,8), \mathrm{KXX1}$

7206

7207

COMMON/CALGOR/O $(30,11,8)$, FXA $(30,11, E), F Y A(30,11,8), F Z A$

7208

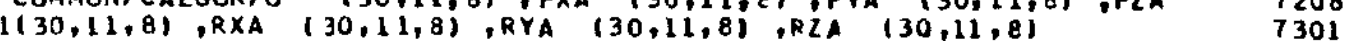

COMMON/SOFXYZ/F 2XA ( $30,11,8)$, F 2YA $(30,11, E), F 22 A(30,11,8)$

COMMON/VAR2/RTX(30,11,8),RTY $(30,11,8), F T \times(30,11,8), F T Y(30,11,1303$

(8),RTL(30,11,8), FTL (30,11,8)

COMMON/DELTS/

COMMON/OELTZ/DELIAZI BI,FRACZ( B)

FRACX, FRACY

$N 5=7 * K N * I N * J M$

$N 6=3 * K N *[N * J M$

CALL CLEAR(O,NS)

CALL CLEAR (F2XA, NG)

CALGULATE SIGMAS, PX, PY, AND PI

FRAC $X=D T / D X$

FRACY $=$ DT / DY

DO $3 K=1, K N$

3 FRACZ $\{K\}=$

DI JELIAZ (K)

CALL SCAYEG

DO $500 K=1, K N$

$005001=2 . I N N$

$00500 \mathrm{~J}=2, \mathrm{JH}$

IF (CII,J,K).LT.L.E-10, GOTC 500

SIGMAX $=U(I, J, K) * F R A C X$

$S I G M A Y=V(I, J, K) * F R A C Y$

SIGMAZ $=W(I, J, K) * F R A C Z(K)$

IF (S IGMAX.NE. O. ISI GNX =SIGMAX/ABSISIGMAX)

IFI SI GMAX.EQ.0.) $51 G N X=1$

IFISIGMAY.EQ.0.) $\leq I G N Y=1$.

I F(SI GMAY . NE . O. I SI GNY = SI GMAY/ABS(SIGMAY)

IF I SIGMAZ .EO. 0. ) \$IGNZ = 1 .

IFISIGMAZ.NE. O. ISI GNZ = SI GMAZ/ABSISI GHAZ)

IF (SIGMAX.GT.0.) GOTO 100

$P X=(-2, *(F I X(1, \therefore, K)+S I G M A X)+R T X(I, J, K)-1, I /(2, * R T X(I, J, K))$ GoT 110

$100 \mathrm{PX}=(2, *(F T X(1, J, K)+S I G M A X)+R T X(I, J, K)-1,1 /(2, * R T X(I, J, K))$

110 IFISIGMAY.GT.0.) GC TC 120

$P Y=(-2, *(F T Y(I, J, K)+S I G M A Y)+R T Y I I, J, K)-1,1 /(2, * R Y Y(I, d, K)]$ GUTU 130

120 PY $=(2, *(F T Y(I, J, K)+S I G M A Y)+R T Y(I, J, K)-1.1 /(2, * R T Y(I, J, K))$

130 IFISIGMAZ.GT.U.) GC TO 140

$P Z=1-2, *(F I Z(I, S, K)+\operatorname{SIGMAZ})+R T Z(I, \downarrow, K)-1,) /(2, * R T Z(I, J, K)$ GUTO 145

$140 P Z=(2, *(F T Z(I, J, K)+5 I G H A Z)+F J Z(I, J, K)-1, I /(2, * R T L(I, J, K)$ C

145 CONTINIUE

NOKMAL ECNOITIIJNS

7304

7305

7306

7307

7308

7401

7402

7403

7404

7405

7406

7407

7408

7501

7502

7503

7504

7505

7506

7507

7508

7601

7602

7603

7604

7605

7606

7607

7608

7701

7702

7703

7704

7705

7736

7707

7708

7801

7802

7803

$78 \mathrm{BC}$

7805

7810

7807

7808

IF $P X, P Y, P Z<U$ CR PX,PY,PZ>I MOCIFICATICAS ARE MADE 7GOI

7902 


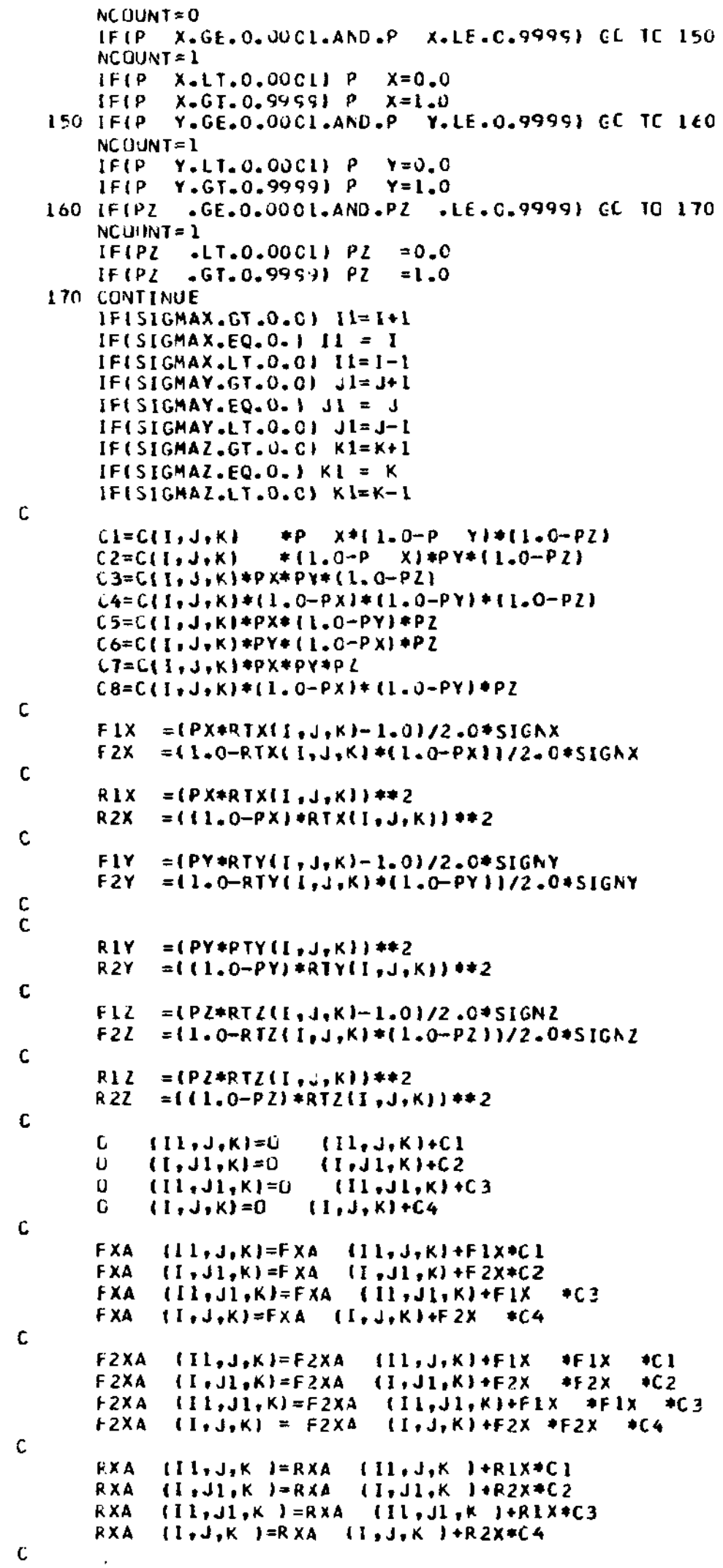




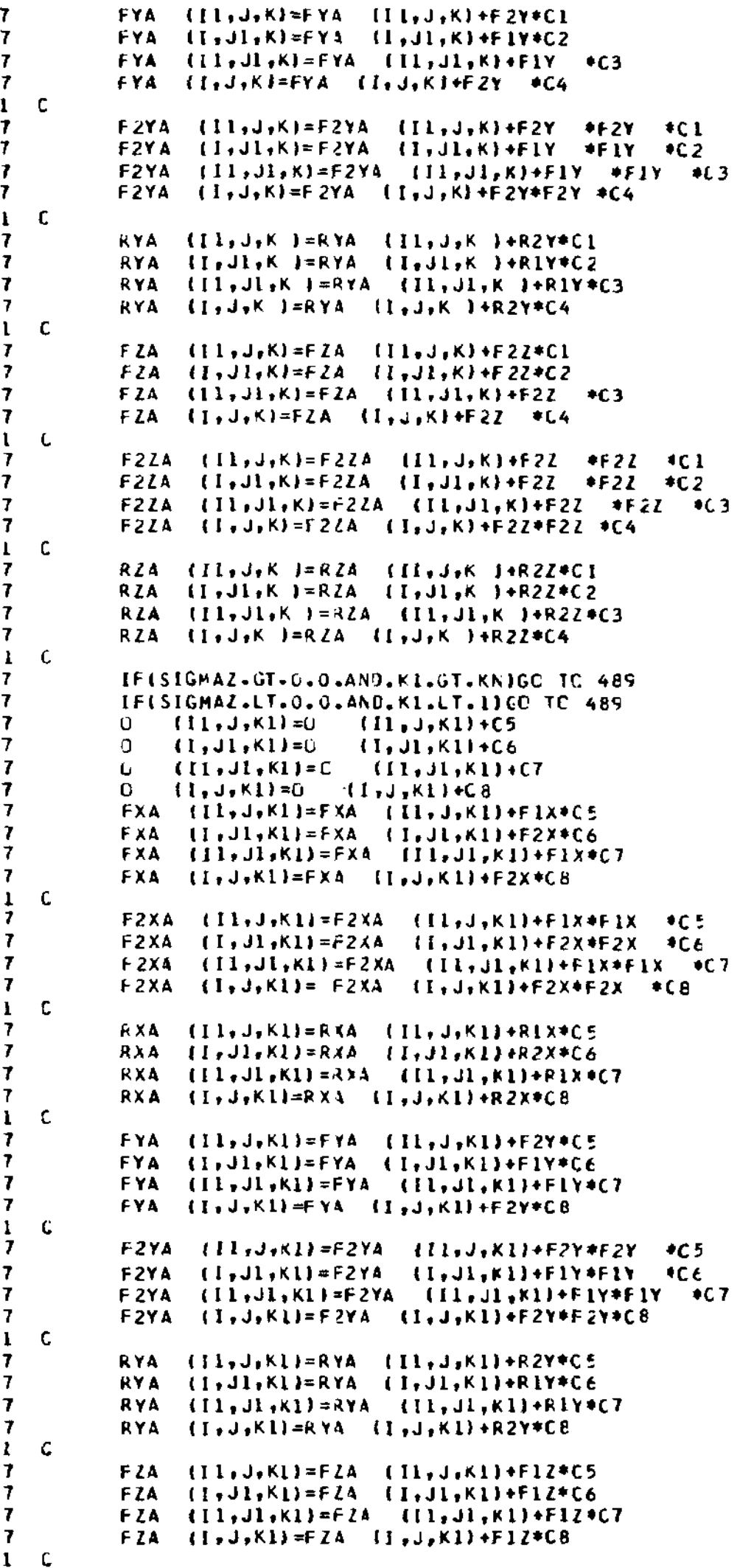

8908

9001

9002

9003

9004

9005

9006

9007

9008

9101

9102

9103

9104

9105

9106

9107

$91 C B$

9201

9202

9203

9204

9205

9206

9207

9208

9301

9302

9303

9304

9305

9306

9307

9308

4401

9402

9403

9404

7405

9406

9407

9408

9501

9502

9503

9504

9505

9506

9507

9508

9601

9602

9603

9604

9605

9606

9607

9608

9701 


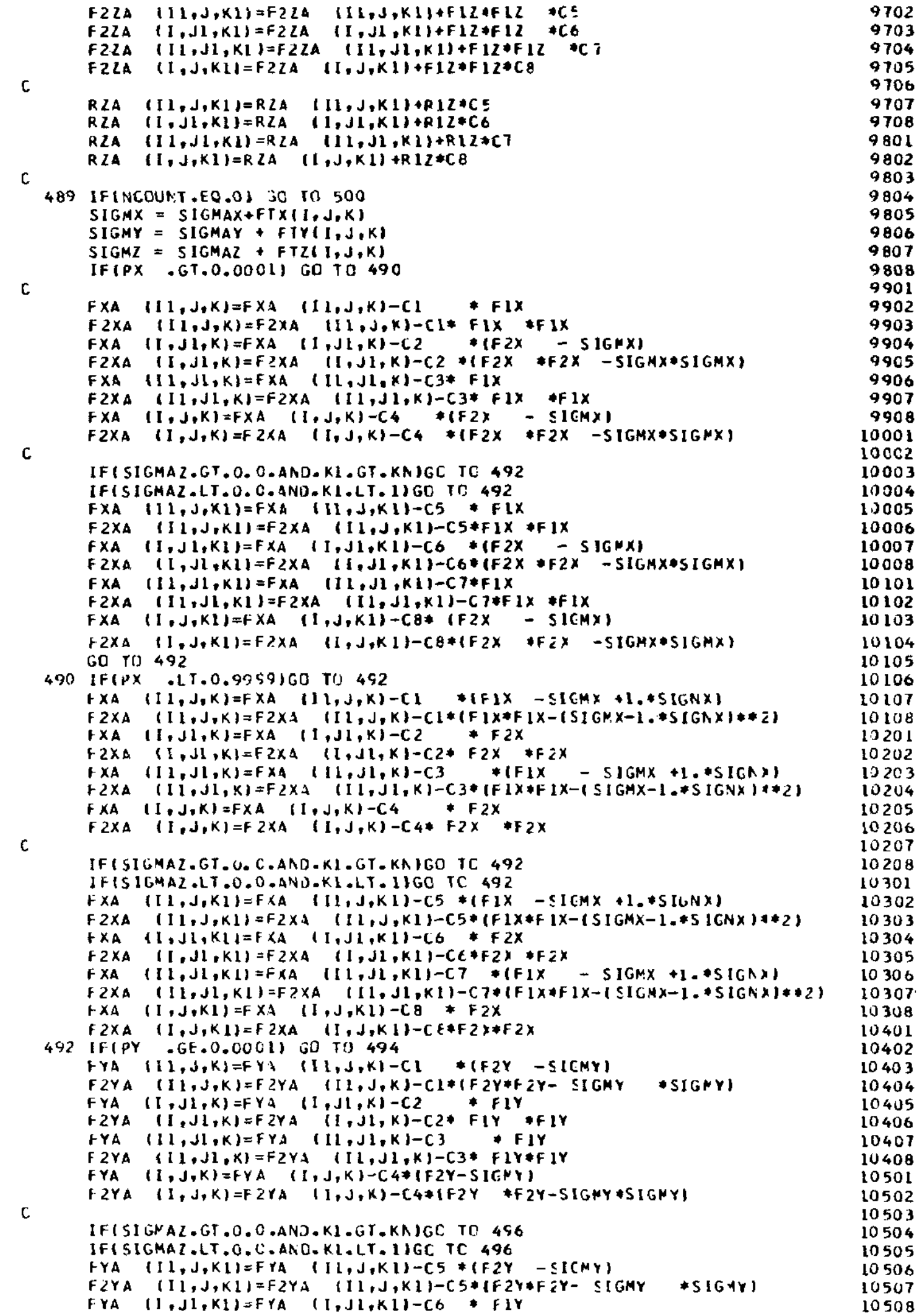




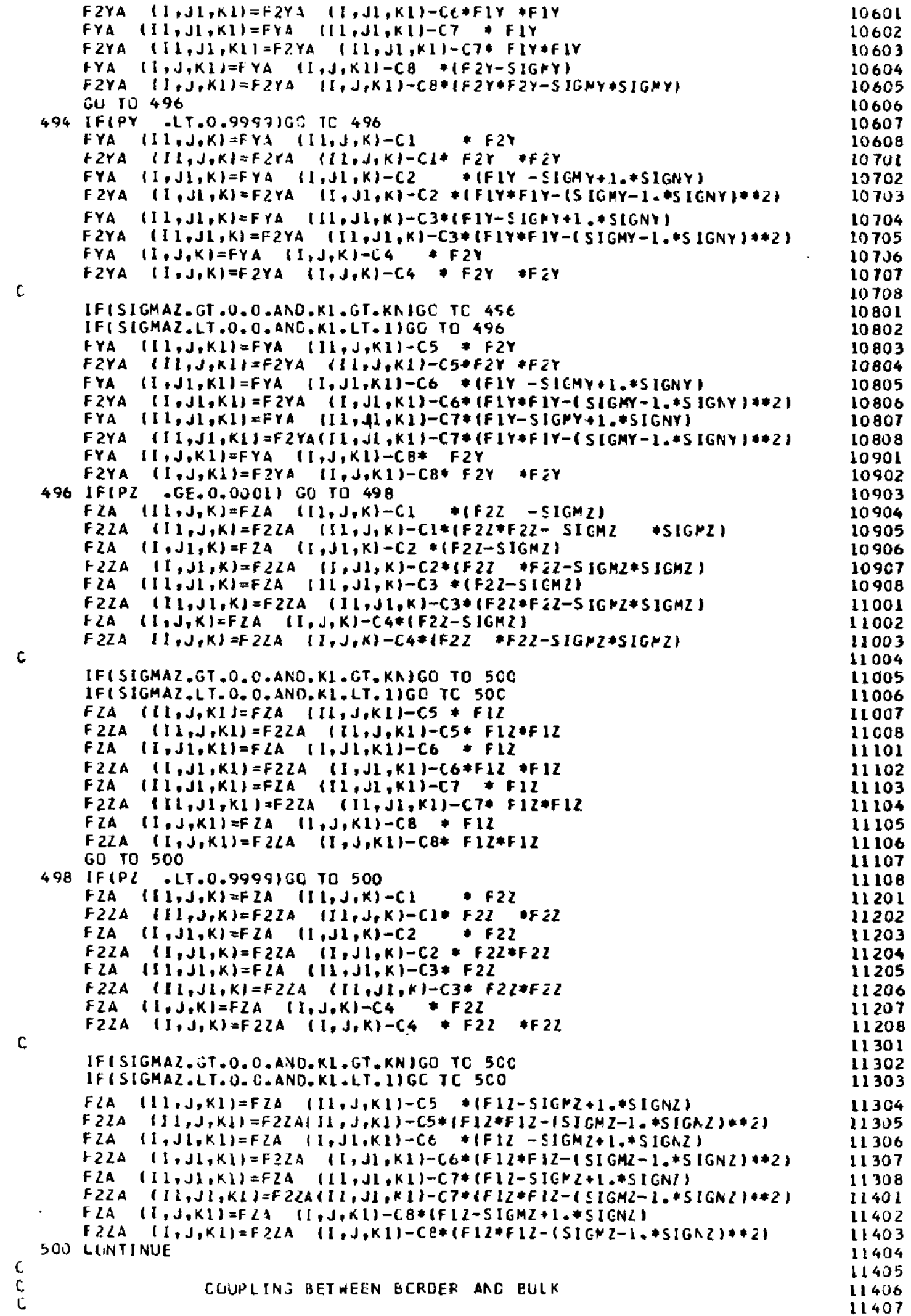




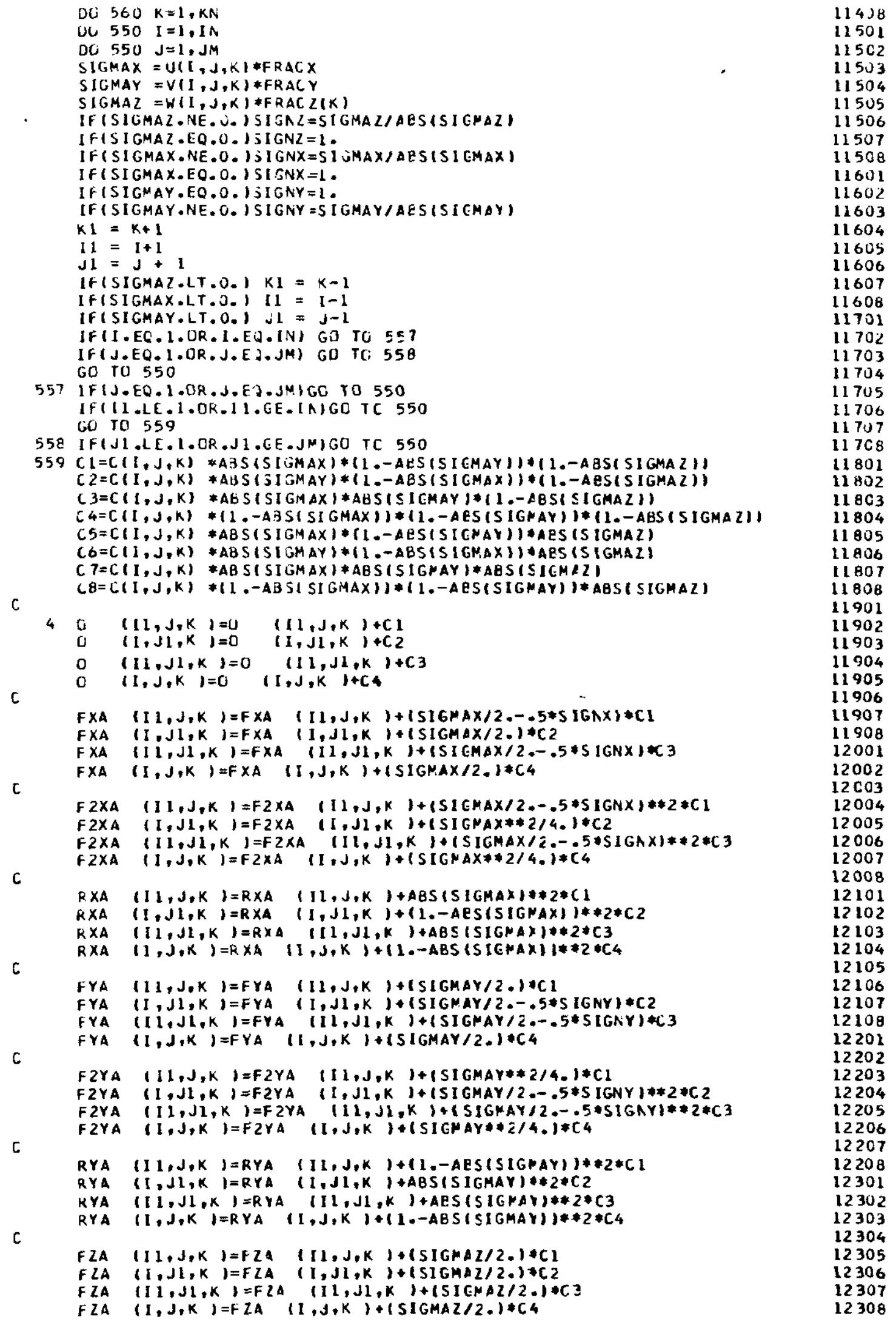




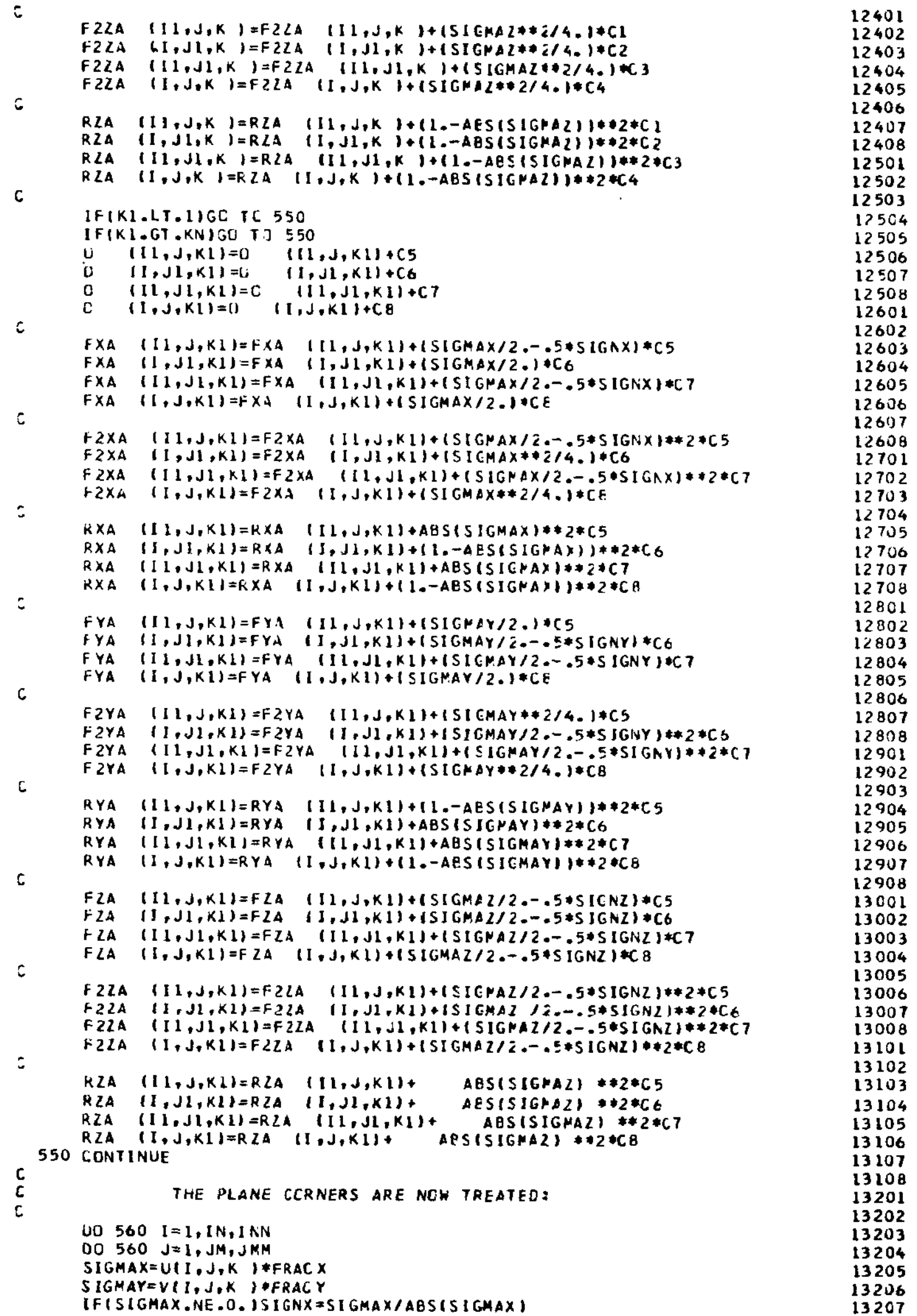

$(11, J, K)=F 2 L A \quad(11, J, K) 1+(51$ EMA $2 * * a / 4,1 * C 1$

F2ZA $\quad I, J 1, K)=F 2 Z A \quad(1, J 1, k)+1 S I G M A 2 * * 2 / 4) * C$.

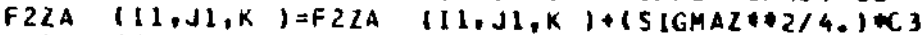

6

F 22 A

(I.J.K I=F ZLA (I,J,K I+ISIGMIL*2/4, I*C4

RLA $(11, J, K)=R Z A(11, d, K)+(1,-A E S(S I G H A Z)) * 2 * C)$

$R 2 A(I, J I, K)=R L A \quad(I, J 1, K)+(1,-A B S(S I G M A Z) i * 2 * C ?$

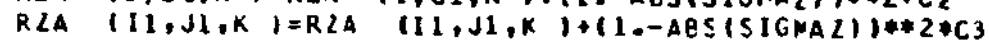

C

$R Z A(I, J, K \quad 1=R Z A \quad(I, J, K)+(1,-A B S(S I G M A Z)) * 2 * C 4$

IFIKI.LT.IIGC TC 550

IFIKI.GT.KNIGOTJ 550

U $\quad(11, J, K 1\}=0 \quad(11, J, K)+C 5$

i) $\quad(1, J 1, K 1)=\mathrm{L} \quad(1, \mathrm{Jl}, \mathrm{K} 1)+\mathrm{C} 6$

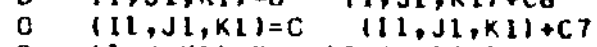

$c$

$(1, J, K(1)=0 \quad(1, J, K 1)+C 8$

FXA $([1, J, K I)=F \times A \quad([1, J, K 1)+(S[G M A X / 2,-.5 * 5 \operatorname{lgA}) * C 5$

FXA $(1, J 1, K 1)=F \times A(1, J 1, K 1)+(5 I G M A X 12)+,C_{6}$

FXA $(I 1, J 1, K())=F \times A \quad(11, J l, K()+(S I G M A X / 2 .-.5 * 5 I G N X) * C 7$

6

FXA $(1, J, K 1)=F \times A \quad(1, J, K 1)+(S I G M A X / 2) * C$,

$+2 \times A \quad(11, J, K 1)=F 2 \times A \quad(I 1, J, K 1)+(S \mid G M A X / 2 .-.5 * 5 I G N \times) * 2 * C 5$

$F 2 \times A \quad(I, J 1, K 1)=F 2 \times A \quad(I, J 1, K 1)+15 I G M A X * 2(4,1)+C 6$

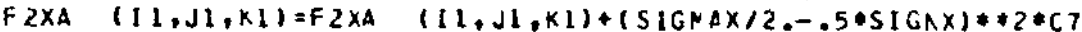

:

r2XA $(I, J, K I)=F 2 \times A(1, J, K I)+(S I G M A X * 214, j * C E$

$K \times A \quad(I 1, J, K I)=R \times A \quad(I L, J, K 1)+A B S(S I G M A X) * 2 *(5$

$R \times A \quad(I, J\{, K I)=R \times A \quad(I, J 1, K(1)+(1,-A B S(S I G+A X)) * * 2 * C 6$

$R \times A \quad(I 1, J 1, K 1)=R \times A \quad(11, J 1, K 1)+A B S(S I G * A X) * * 2 * C 7$

こ

$R \times A \quad(I, J, K I)=R \times A \quad(I, J, K I)+(1,-A B S(S I G N A) I) * 2 * C B$

FYA $(I 1, J, K 1)=F Y A(I 1, J, K 1)+(S I G K A Y / 2) *,(5$

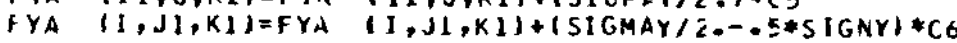

FYA $(11, J l, K 1)=F Y A(11, J 1, K 1)+(S I G M A Y / 2 \ldots-5 * S I G N Y) * C 7$

$c$

FYA $(1, J, K 1)=F Y A(I, J, K 1)+(S I G M A Y / 2,1 * C E$

F2YA $(I 1, J, K 1)=F 2 Y A \quad(I 1, J, K 1)+(S I G M A Y * * 2 / 4,1)$ CS

F2YA $(1, J 1, K 1)=F 2 Y A \quad(1, J 1, K 1)+1$ SICMAY $2,-.5 * 5$ JGIYY) **2*CS

$F 2 Y A(I 1, J 1, K 1)=F 2 Y A(I), J 1, K 1)+(S I G M A Y, 2,-.5 * S I G N Y) * 2 * C 7$

c

F 2YA

$(I, J, K L)=F 2 Y A \quad(I, J, K 1)+(S I G M A Y * 214) * C$,

RYA $(I 1, J, K I)=R Y A(I 1, J, K I)+(1,-A B S(S I G M A Y)) * 2 * C 5$

RYA $(I, J 1, K 1)=R Y A(I, J 1, K 1)+A B S(S I G N A Y) * * 2 * C 6$

RYA $(11, J 1, K 1)=R Y A(1, J 1, K 1)+A B S(S I G M A Y) * 2 * C 7$

RYA $(I, J, K I)=R Y A(I, J, K I)+(1,-A E S(S I C M A Y) * * 2 * C B$

6

FZA $(11, J, K L)=F Z A \quad(I 1, J, K 1)+(S[G M A Z / 2 .-.5 * S[C N Z) * C 5$

$F Z A(J, J 1, K 1)=F 2 A(J, J 1, K 1)+(S I G M A 2 / 2,-.5 * S J G N Z) * C 6$

F ZA $(I 1, J 1, K 1)=F Z A(I 1, J 1, K I)+(S I G N A Z 12 .-.5 * S[G N Z) * C 7$

$c$

$F L A \quad(1, J, K 1)=F Z A \quad(I, J, K L)+(5 I G M A Z 12,-.5 * 5 I G N Z) * C 8$

F $2 Z A(I 1, J, K 1)=F 2 L A \quad(I 1, J, K 1)+(\$ I C H A Z / 2,-.5 * S I G N Z) * 2 * C 5$

F22A $(1, J 1, K()=F 22 A \quad(I, J 2, K 1)+1 S I G M A I, 2=-5 * 5 \mid G N 21 * * 2 * C 6$

F22A $(I 1, J 1, K 1)=F 2 Z A(I 1, j 1, K 1)+15 I G M A Z 12,-5 * 5 I G N Z) * 2 * C 7$

:

FLLA $(1, J, K L)=F 2 Z A(1, J, K 1)+(S I G M A Z I Z+-5 * S I G N Z)+2 * C B$

RLA $(I 1, J, K L)=R Z A \quad(I 1, J, K I)+\quad A B S(S I G F A Z) * 2 * C 5$

RZA $(1, J 1, K L)=R Z A \quad(1, J 1, K 1)+\quad A E S(S I G P O Z) * 2 * C 6$

RZA $(11, J 1, K 1)=R 2 A(I 1, J 1, K 1)+\quad A B S(S I G M A Z) * * 2 * C 7$ 550 CONTINUE

RZA $(I, J, K I)=R Z A(I, J, K I)+$ APS $(S I G M A Z) * 2 * C 8$

c

6

THE PLANE CCRNERS ARE NGH TREATED:

Uo $560 \mathrm{I}=1, I N, I N N$

DO $560 J=1, J M, J M M$

SIGMAX =UII,J,K I*FRAC $X$

SIGMAY $=V I I, J, K$ J OFRACY

IFISIGMAX.NE.0. ISIGNX =SIGMAX/ABS(SIGMAX)

12404

12405

12406

12407

12408

12501

12502

12503

12504

12505

12506

12507

12508

12601

12602

12603

12604

12605

12606

12607

12608

12701

12702

12703

12704

12705

12706

12707

12708

12801

12802

12803

12804

12805

12806

12807

12808

12901

12902

12903

12904

12905

12906

12907

12908

13001

13002

13003

13004

13005

13006

13007

13008

13101

13102

13103

13104

13105

13106

13107

13108

13201

13202

13203

13204

13205

13206

13207 


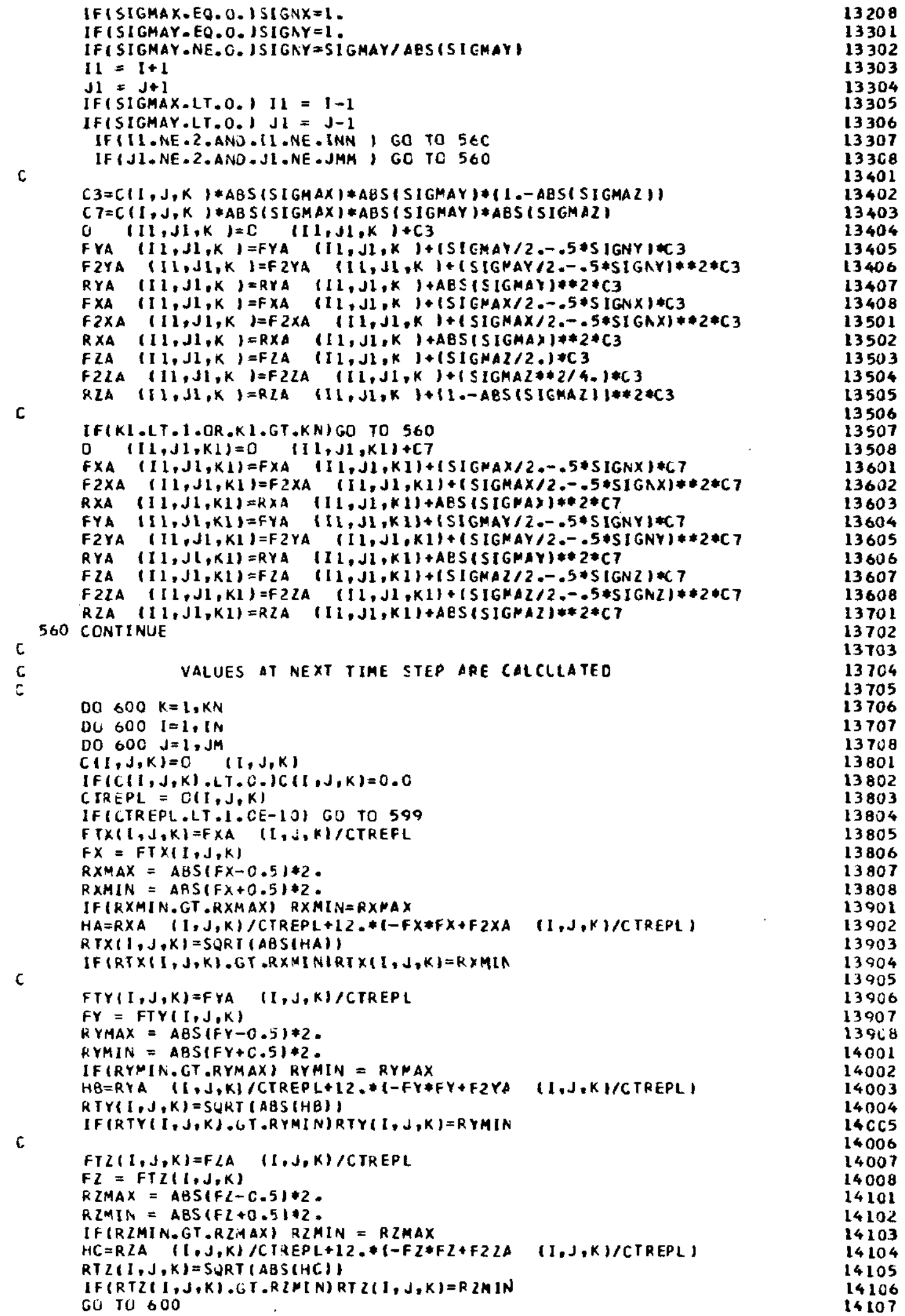




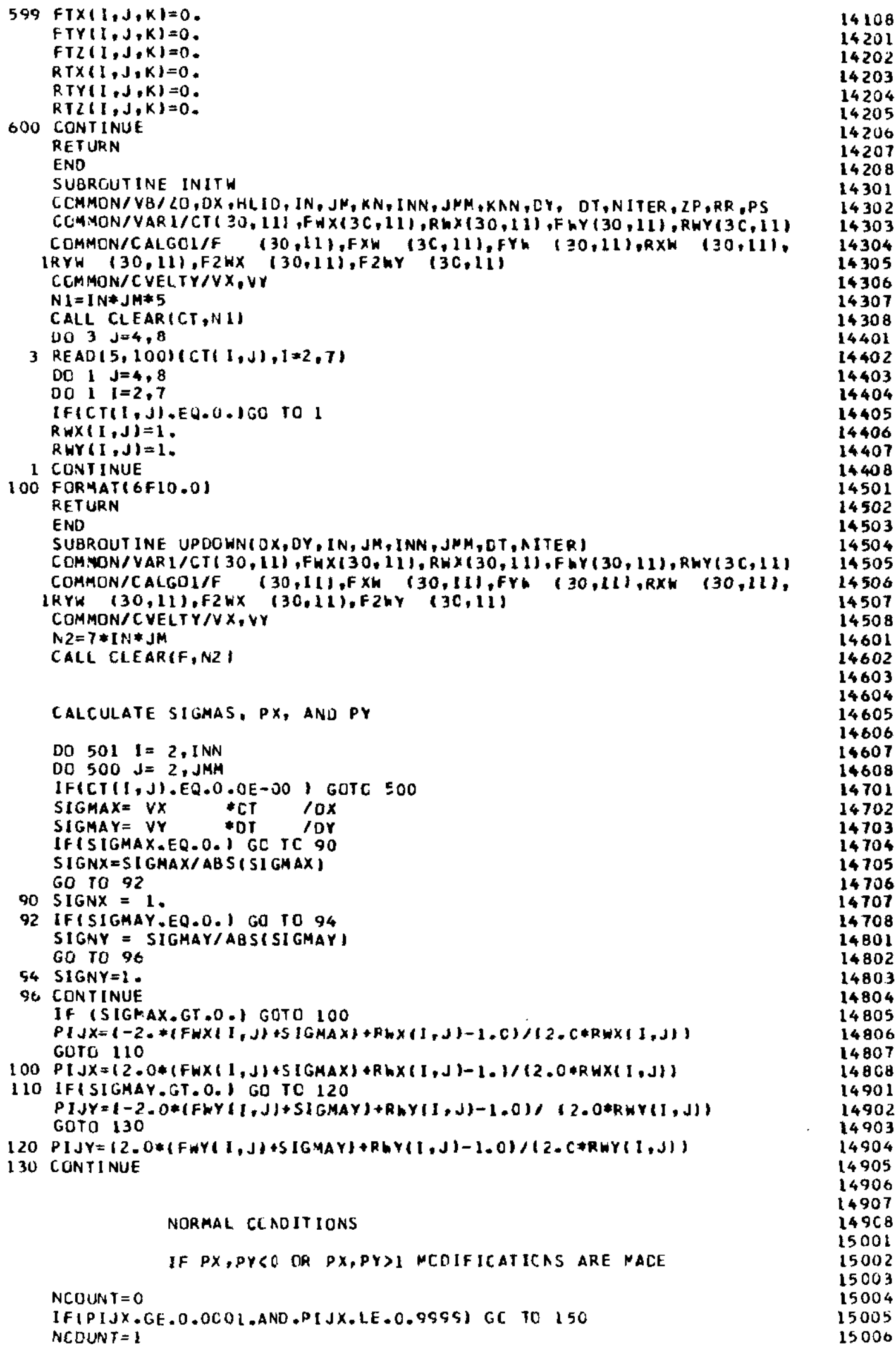

15004

15005

15000 


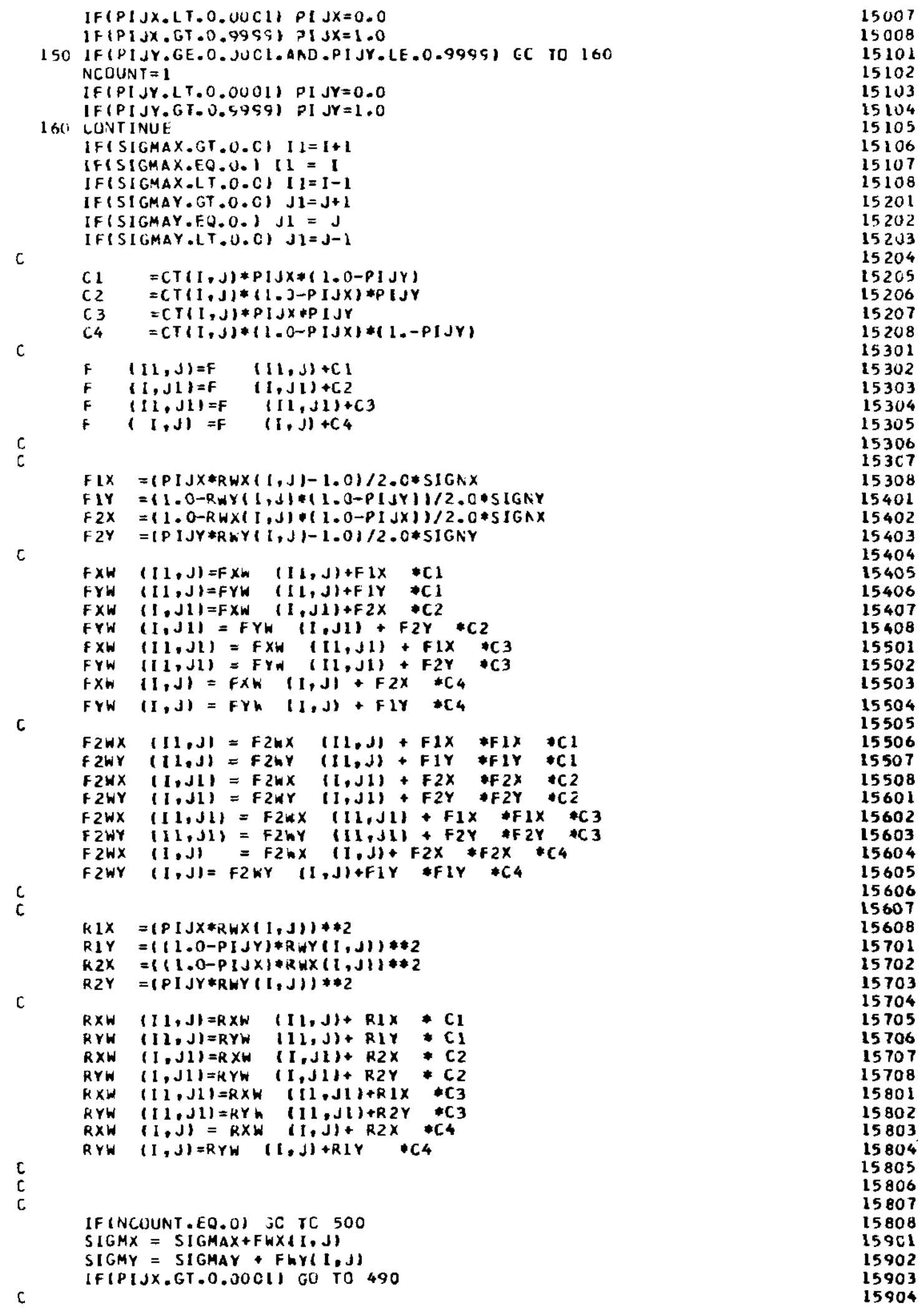




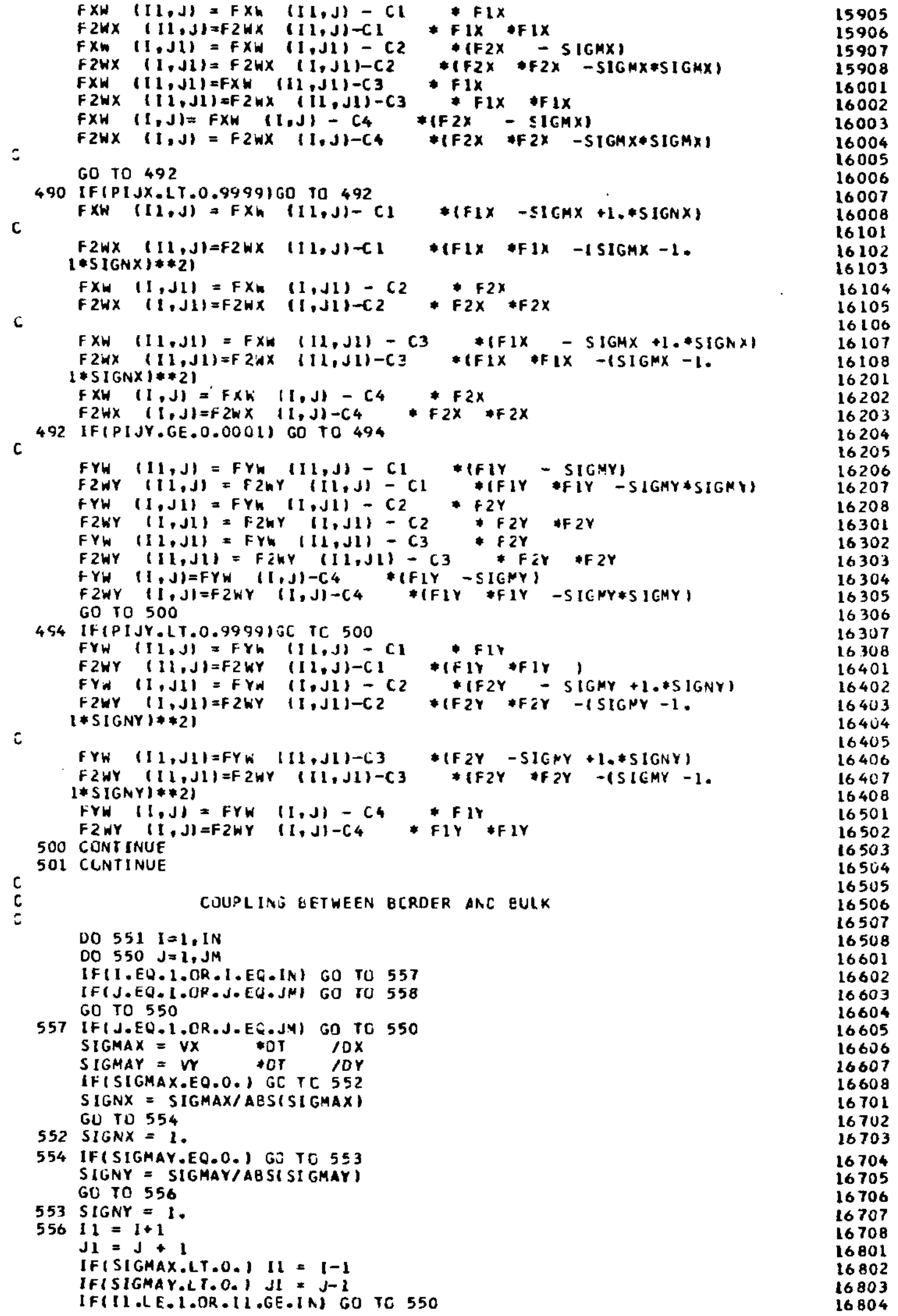

16004
16005

16006

16007

16008

16101

16102

16103

16104

16105

16106

16107

16108

16201

16202

16203

16204

16205

16206

16207

16208

16301

16302

16303

16304

16305

16306

16307

16308

16401

16402

16403

16404

16405

16406

16407

16408

16501

16502

16503

16504

16505

16506

16507

16508

16601

16602

16603

16604

16605

16606

16607

16608

16701

16702

16703

16704

16705

16706

16707

16708

16801

16802

16803

16804 
GC TO 559

558 IF (I.LE.I.I:R.I.GE.IA) GO TO $5 \leq 0$

SIGMAX $=V X$ OOT IOX

SIGMAY = VY *CT IDY

IFISIGMAX.EQ.0.) GC IC 5520

SIGNX = SIGMAX/ARS(SIGMAX)

GO TO 5540

5520 SIGNX $=1$.

5540 IFISIGMAY.EQ.0. I GO TO 5 E30

SIGNY = SIGMAYIABSISI GMAY)

GO TO 5560

5530 SIGNY $=1$

$5560 I_{1}=1+1$

$J 1=J+1$

IFISIGMAX.LT .0.)II $1=1-1$

IF (SIGMAY.LT.0.) JI $=\mathrm{J}-1$

IF(J1.LE.1.OR.JI.GE.JM) GO TO 550

$559 C_{1}=C T(I, J) * A B S(S I G M A X) *(1$.-AES (SIGMAY))

$C 2=C T(I, J) * A B S(S I G M A Y) *(1 .-A B S(S I G$ GXX) $)$

$C 3=C T(I, J) * A B S(S I G M A X) * A B S(S I G M A Y)$

c

$C_{4}=C T(1, J) *(1 .-A B S(S I G M A X)) *(1 .-A E S(S I G M A Y))$

16805

16806

16807

16808

16901

16902

16903

16904

16905

16906

16907

16908

17001

17002

17003

17004

17005

17006

17007

17008

17101

17102

$F \quad(11, J)=F \quad(11, J)+C_{1}$

$+\quad(I, J 1)=F(1, J 1)+C_{2}$

$F \quad(I l, J 1)=F \quad(11, J 1)+C 3$

C

$F \quad(I, J)=F \quad(I, J)+C_{4}$

17103

17104

17105

17106

17107

$F X W(I 1, J)=F X W(I 1, J)+(S I G M A X / 2,-C .5 * \operatorname{SIGNXI*C1} \quad 17108$

FYW $(I 1, J)=F Y M \quad(I 1, J)+(S I G M A Y / 2, j * C 1$

$F X W(I, J 1)=F X W \quad(1, J 1)+(S I G \operatorname{Max} / 2) * C$,

FYW $(I, J 1)=F Y W(1, J 1)+(S I G M A Y / 2,-C .5 * S I G N Y I * C 2$

$F X W(I 1, J 1)=F X W(I 1, J 1)+(S I G M A X / 2,-C, 5 * S I G N X) * C 3$

17201

17202

17203

17204

FYW $(I 1, J 1)=F Y A(I 1, J 1)+($ SIGMAY $/ 2 .-0.5 * 5 I G N Y) * C 3$

FXH $([, J)=F X H(I, J)+(S I G M A X / 2) * C 4$,

6

FYH $(1, J)$ *FYh $(1, J)+(S I G M A Y / 2), * C_{4}$

17206

17207

17208

17301

17302

17303

F2WY $(1, j J)=F 2 W Y \quad(11, J)+($ SIGMAY**2/4.)*C1

F $2 W X(1, J 1)=F 2 W X(1, J 1)+($ SIGMAX**2/4.) \#C 2

F 2hY $(1, J 1)=F 2 h Y(1, j 1)+(S 1 G H A Y / 2 .-C .5 * 51 G N Y) * 2 * C 2$

FWWX $(I 1, J 1)=F 2 W X(11, J 1)+($ SIGMAX/2,-C.5*SIGNX)*2*C3

17304

17305

F $2 W Y(I 1, J 1)=F 2 W Y(11, J 1)+(S I G H A Y / 2,-C .5 * S I G N Y) * 2 * C 3 \quad 17306$

$F 2 W X(1, J)=F 2 N X(I, J)+(\operatorname{SIGMAX} * 2 / 4). * C_{4}$

c

$F Z W Y(1, J)=F 2 W Y(I, J)+(S I G M A Y * 2 / 4) * C$,

17307

17308

$R \times W(11, J)=R \times W(11, J)+A B S(S I G M A X) * 2 * C l$

RYW $(I 1, J)=R Y M(I 1, J)+(1 .-A B S(S I C M A Y)) * 2 * C 1$

$R \times H(I, J 1)=R \times W(I, J 1)+(1 .-A B S(S I G M A X)) * 2 * C 2$

RYW $(I, J 1)=R Y M(I, J 1)$ + AES(SIGMAY) Q Z EC 2

RXW $(11, J 1)=R W_{W}(11, J 1)+A B S(S I G M A X) * 2 * C 3$

$R Y h(I 1, J 1)=R Y W(I 1, J i)+A B S(S I G N A Y) * 2 * C 3$

$R \times h \quad(I, J)=R \times K^{\prime}(I, J) *(1,-A B S(S I G M A X)) * 2 * C 4$

RYW $(1, J)=$ RYh $(1, J)+(1,-$ ABS $(S$ IG*AY $) * 2 * C 4$

550 CONIINUF

6

551 CONT I NUE

6

THE FGUR CCRNERS ARE NCW TREATED:

DOI $561 I=1, I N, I A N$

DC $560 \mathrm{~J}=1, J M, J P M$

SIGMAX $=V X \quad$ *DT $\quad / D X$

SIGMAY = VY TOI NOY

IFISIGMAX.EQ.0.) GO TO 562

SIGNX = ABSISIGNAXI/SIGMAX

GL: TO 564

562 SIGNX $=1$.

564 IFISIGMAY.EQ.0.1 GC TG 563 SIGNY = ABS ISIGRAY)/SIGMAY GL TO 566

$5 E 3$ SIGNY $=1$.

$56611=1+1$

$J 1=J+1$

17401
17402

17403

17404

17405

17406

17407

17408

17501

17502

17503

17504

17505

17506

17507

17508

17601

17602

17603

17604

17605

17606

17 6u 7

17608

17701

17702

17703

17704 


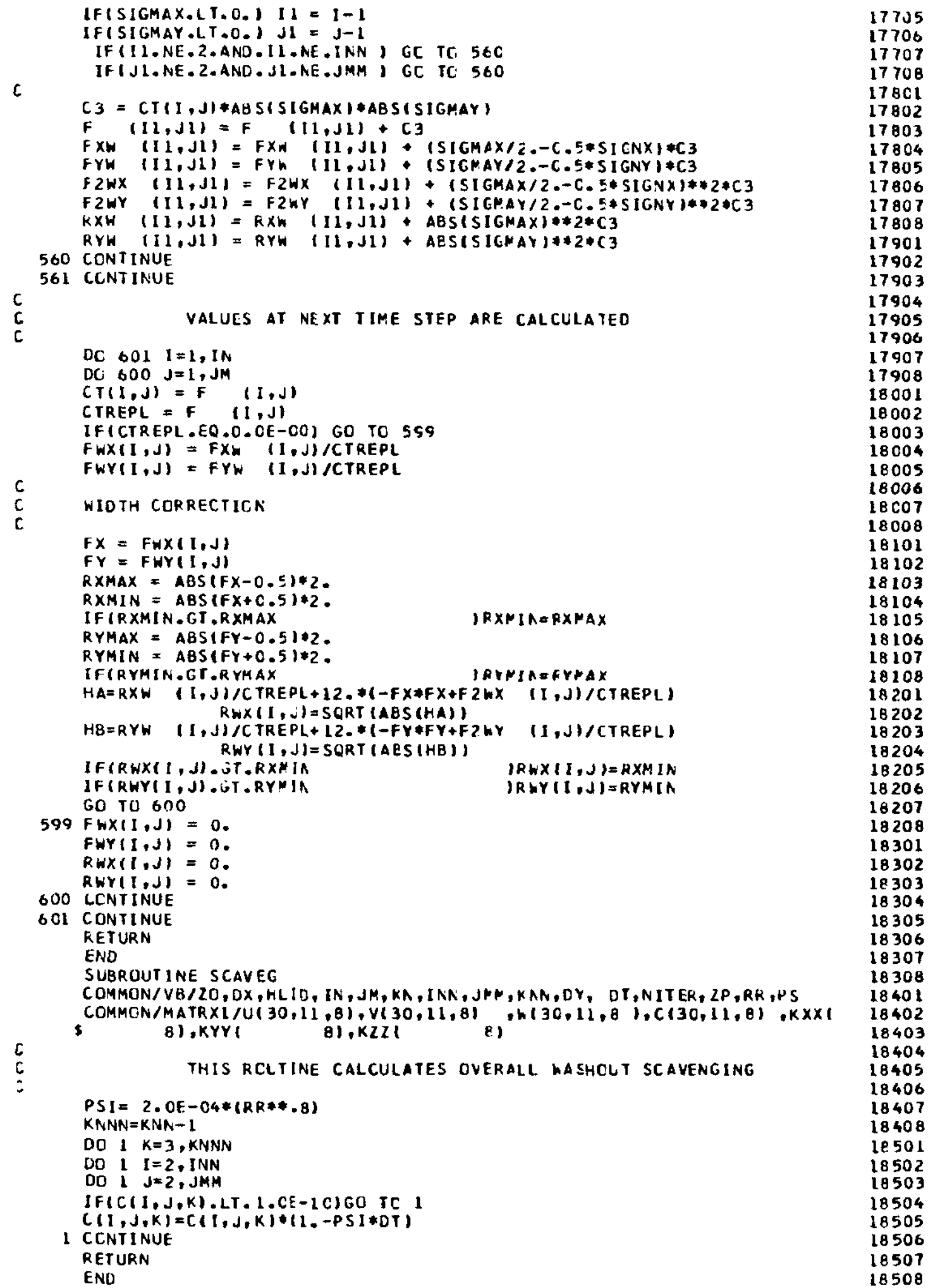


SLBROUTINE INITV

CLMMON VBIZO ,OX,HLIO,IN,JM, KN,INN,JHN,KAN,OY, OT,NITER, ZP,RR,PS 1.2

CCMMON $V A R V / C V(30,11), F V X(3 C, 11), R \cup X(30,1), F \vee Y(30,11), R \cup Y(3 C, 11) \quad 1,3$

COMMON/CALGOV/FV $(30,11)$,FXV 130,11$)$, FYV $(30.11), R X V(3 C, 111,1.4$

IPYV $(30,11), F 2 V X(30,11)$,F2VY $(3 \mathrm{C}, 11)$

CLMMUN/CVELTYIVX, VY 1.6

$N 2=I N * J M * T$

CALL CLEAR(CV,NI)

CALL CLFARIFV,N2)

DO $3 \mathrm{~J}=4,8$

DO $3 \quad I=3,7$

$3(V(I, J)=($ FLCAT $(I)-5) * 5.0$.

DO $1 \mathrm{~J}=4,8$

DO $11=3,7$

If(CVII,J).EQ.0.160 IC I

$\operatorname{R\cup X}([, J)=1$.

$R \vee Y(1, J)=1$.

I CONTINUE

RETUIN

ENO

SUBRTIUT INE LATERVICX, CY, IN, JM, INN, JHM, DT, MITER)

COMMON/ VAR V/CV(30, 11$), F \vee X(30,11), \operatorname{RVX}(30,1), F \cup Y(30,11), \operatorname{RVY}(3 C, 11)$

COMMON CALGOVIFV $(30,11), F \times V(30,11), F Y\}(30,11), R \times V(30,11)$,

LRYV $(30,11)$, F $2 V \times(30,11)$, F 2 VY $(30,11)$

CUMM JN/CVELTY/VX, WY

$N 2=7 * I N * J M$

CALL CLEARIFV,N2)

c

c

c

CAlCulate sigmas, PX, and py

DO $501 \quad I=2, I N N$

$00500 \mathrm{~J}=2, \mathrm{JMM}$

IFICVII,J).EG.0.0E-00, GOTO 500

SIGMAX $=V X$ OOT $/ D X$

SIGMAY= VY *OI IOY

IFISIGMAX.EQ.0.) GO TC 90

SIGNX = SIGMAX/ABS $(S I G M A X)$

GO TO 92

90 SIGNK $=1$.

92 IFISIGMAY.EQ.0.) GC TC 94

SIGNY = SIGMAY/ABS(SIGPAY)

GO TO 96

S4 SIGNY $=1$.

96 CONTINUE

IF (SIGMAX.GT.0.) GOTC 100

PI $J X=(-2 . *(F \vee X(1, J)+S I G M A X)+R V X(I, J)-1.0) /(z, C * R V X(1, J))$

GOTO 110

$100 P I J X=(2,0 *(F \vee X(I, J)+S I G M A X)+R V X(I, J)-1, J) /(2,0 * R \vee X(I, J))$

110 IF(SIGMAY,GT O,) GO TC 120

$P I J Y=(-2.0 *(F V Y(1, J)+S I G M A Y)+F \cup Y(I, J)-1.0) /(2.0 * R V Y(I, J))$

GoTO 130

120 PIJY $=(2.0 *(F V Y(I, J)+5 I G M A Y)+R V Y(I, J)-1.0) /(2,0 * R V Y(I, J))$

130 CINTINUE

c

c

c

$c$

NORMAL CCAOITIONS

IFYPX,PY<C OR PX,PY>I MCDIFICATICAS ARE MADE

NLOUNT $=0$

IF(PIJX.GE.0.0001.AND.PIJX,LE.0.9999) GC IC ISC

NCOUNT $=1$

IFIPIJX.LT.0.0001) PIJX $=0.0$

IFIPIJX.GT.0.9999) PI JX $=1.0$

150 IF(PIJY,GE.O.COCI.AND.PIJY.LE.C.SSSS) GC IS 160

NCOUNT $=1$

IF(PIJY.LT $.0 .00 \mathrm{Cl})$ PI JY $=0.0$

IF(PIJY.GT.0.99SS) PI JY=1.0

160 CLNTINUE

1.7

1.8

2.1

2.2

2.3

2.4

2.5

2.6

2.7

2.8

3.1

3.2

3.3

3.4

3.5

3.6

3.7

3.8

4. 1

4.2

4.3

4.4

4.5

4.6

4.7

4.8

5.1

5.2

5.3

5.4

5.5

5.6

5.7

5.8

6.1

6.2

6.3

6.4

6.5

6.6

6.7

6.8

7.1

7.2

7.3

7.4

7.5

7.6

7.7

7.8

B. 1

8.2

6. 3

8.4

8.5

8.6

8.7

8.8

9.1

9.2

9.3

9.4

9.5

9.6

9.7 


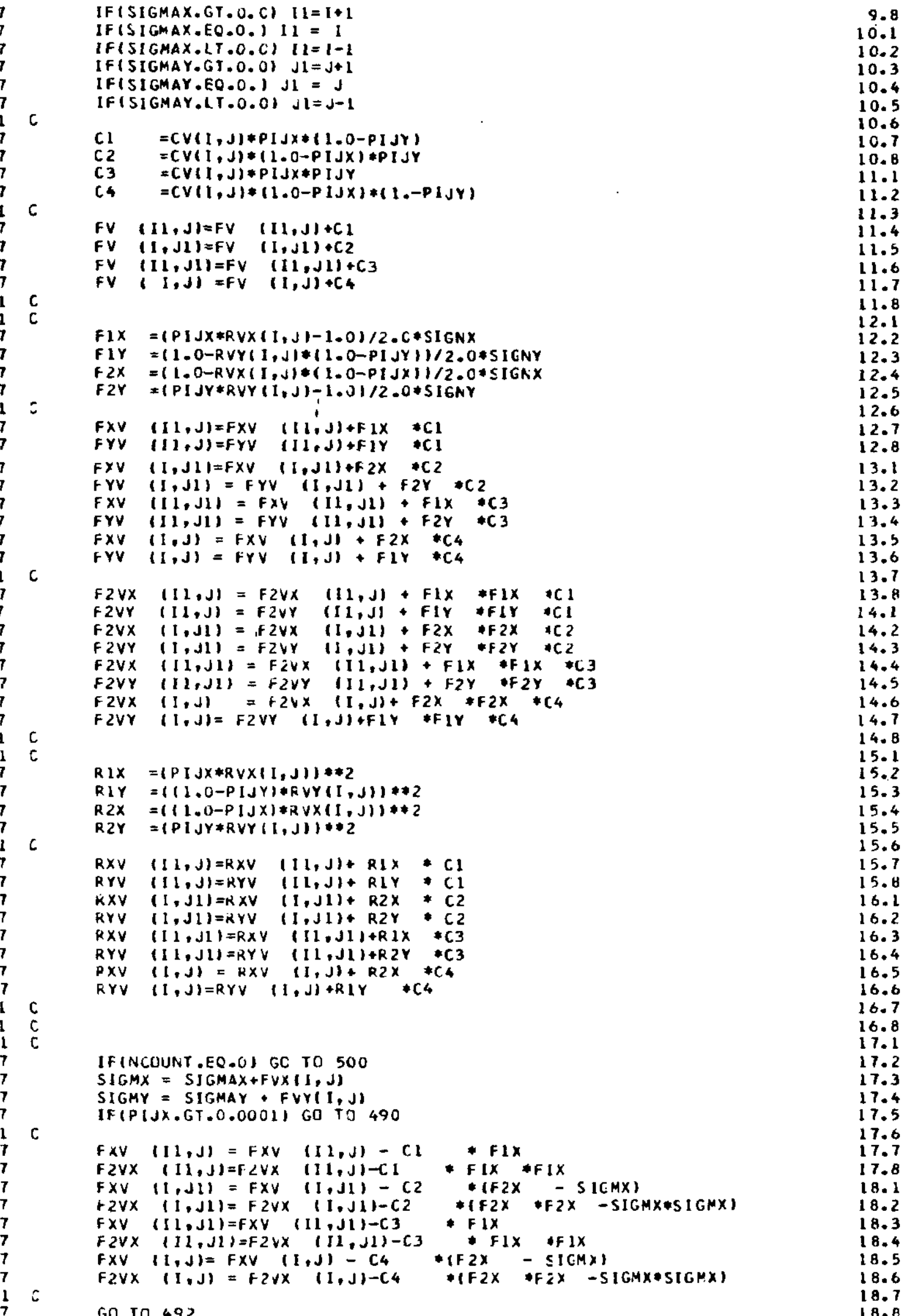




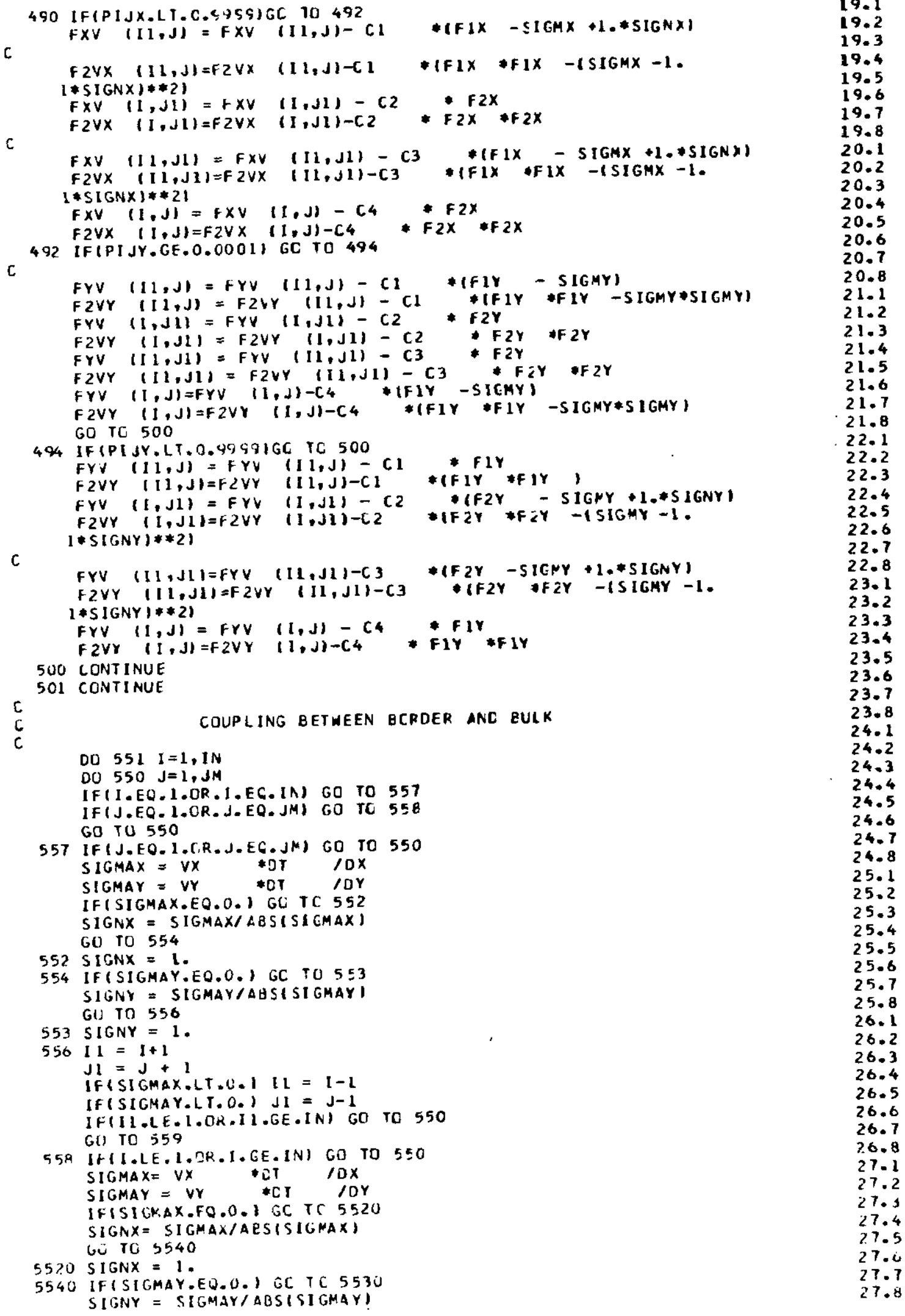




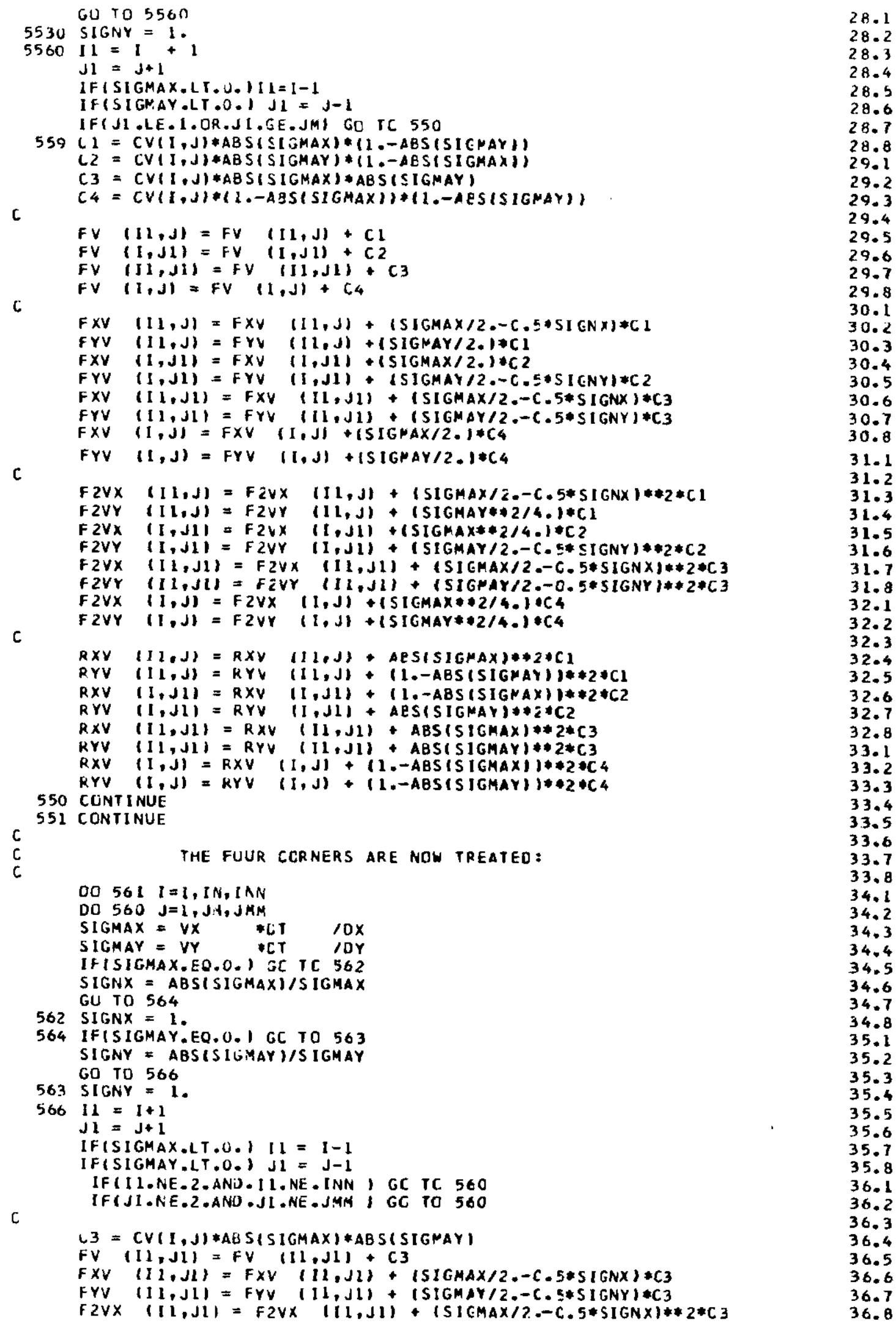

28.1

28.2

28.3

28.4

28.5

28.6

28.7

28.8

29.1

29.2

29.3

29.4

29.5

29.6

29.7

29.8

30.1

30.2

30.3

30.4

30.5

30.6

30.7

30.8

31.1

31.2

31.3

31.4

31.5

31.6

31.7

31.8

32.1

32.2

32.3

32.4

32.5

32.6

32.7

32.8

33.1

33.2

33.3

33.4

3.3 .5

33.6

33.7

33.8

34.1

34.2

34.3

$34-4$

34.5

34.6

34.7

34.8

35.1

35.2

35.3

35.4

35.5

35.6

35.7

35.8

36.1

36.2

36.3

36.4

36.5

36.6

36.7

36.8 


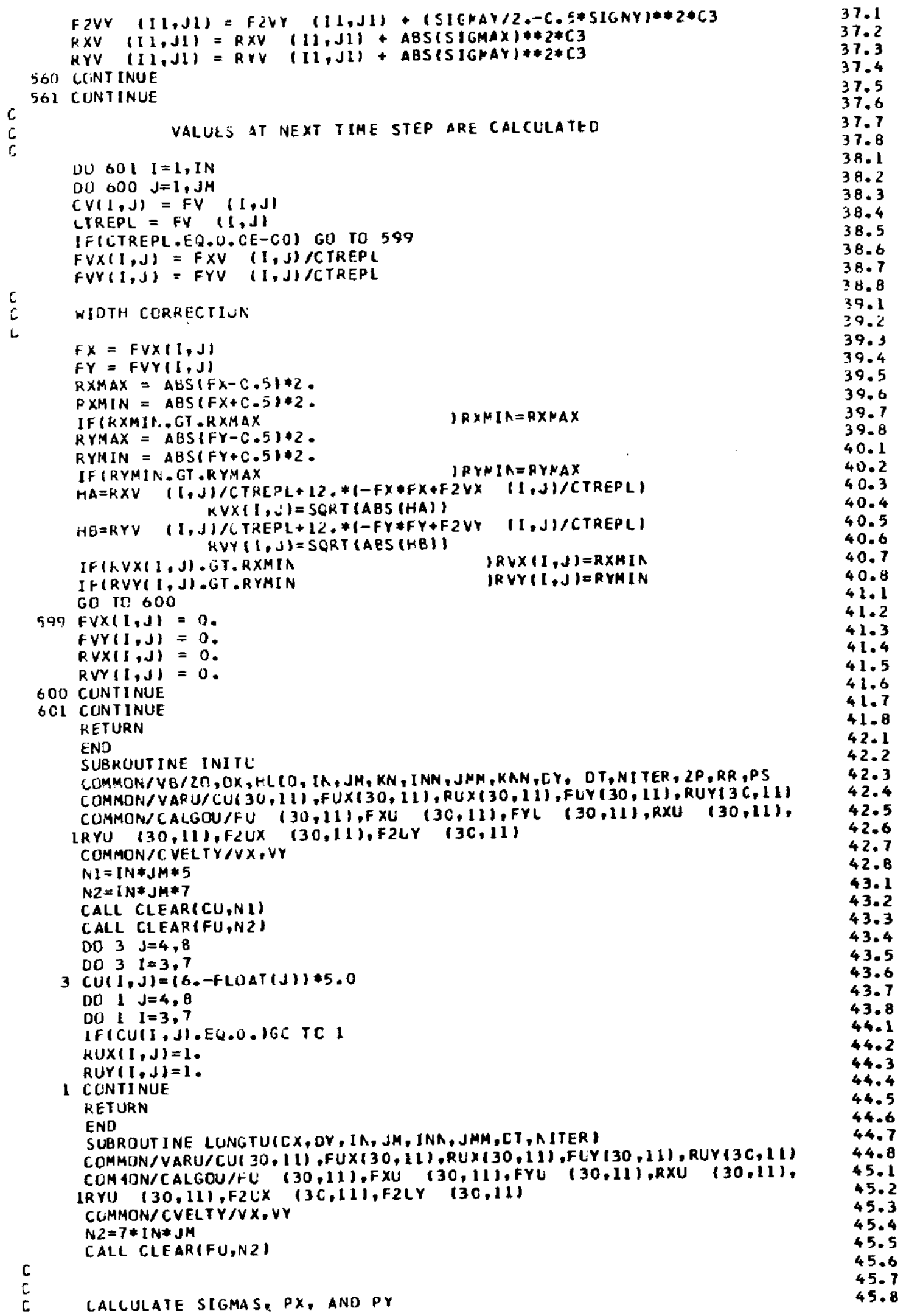

39.5

39.6

39.7

39.8

40.1

40.2

40.3

40.4

40.5

40.6

40.7

40.8

41.1

41.2

41.3

41.4

41.5

41.6

41.7

41.8

42.1

42.2

42.3 
DD $501 \mathrm{I}=2, \mathrm{INN}$

DD $500 \mathrm{~J}=2, \mathrm{JMM}$

IFICUII, J).EQ.0.0E-00, GOTO 500

SIG:AX $=V X$ OD $\quad$ TOX

SIGMAY $=$ VY FOT JOY

IFISIGMAX.EQ.0.1 GO TO 90

SIGNX $=$ SIGMAX/ABS S( SIGMAX)

GO TO 92

90 SIGNX $=1$.

92 IF(SIGMAY.EO. 0.) GC TC 94

SIGNY = SIGMAY/ABSISIGMAY)

GO TO 96

94 SIGNY $=1$.

96 CONT INUE

IF (SIGMAX.GT.0.) GCTC IUO

$P[J X=(-2 * *(F U X(1, J)+S I G M A X)+R(X X(I, J)-1.0) /(2,0 * R U X(I, J))$ GOTO 110

1 Co $P[J X=(2,0 *(F U X(I, J)+S I G M A X)+R(X(I, J)-1) /,(2,0 * R(X X(I, J))$

110 IFISIGMAY.GT.0.1 GO TO 120

PIJY $=(-2.0 *(F U Y(I, J)+S I G M A Y)+F U Y(I, J)-1.0) /(2 . C * R(Y(I, J))$ GUTO 130

120 PIJY $=(2$. O* (FUYI I,JI +SIGHAY $+R C Y(I, J)-1.0) /(2, C * R U Y I I, J))$

130 CONTINUE

c

C

c

c

NORHAL CCADITIONS

IFUPX,PYSC CR PX,PY>I HCEIFICAT ICAS ARE MADE

NCOUNT $=0$

IF (PIJX.GE.0.0001.AND.PIJX.LE.C.9999) GE TC 150

NCOUNT $=$ l

IF\{PIJX.LT.0.0001) PIJX $=0.0$

IF(PIJX.GT.0.995S) PI JX $=1.0$

150 IF(PIJY.GE.0.000I.AND.PIJY.LE.C.9GGS) GC TC 1 tO NCUUNT $=1$

IF (PIJY.LT.O.LUCI) PI JY=0.0

IF(PIJY.GT.0.95\$9) PI JY=1.0

160 CONTINUE

IF(SIGMAX.GT.0.C) $(1=I+1$

IFISIGMAX.EO.0.1 II =

IF (SIGMAX,LT.0.C) $11=1-1$

IF (S I GMAY.GT .0.0) $J 1=J+1$

IH (SIGMAY.EQ.0.) Jl $=J$

$i$

If $(S I G M A Y . L T * 0 . C) J l=J-1$

$C I=(U U(I, J) * P I J X *(1,0-P I J Y)$

$\left[\begin{array}{ll}L^{2} & =\mathrm{CU}(I, J) *(1.0-P I J X) * P I J Y\end{array}\right.$

(3) 3 =CU(I,J)*PIJX*PIJY

C

$C_{4} \quad=C U(I, J) *(1.0-f I J X) *(1,-P I J Y)$

FU $\quad(I 1, J)=F U \quad(I 1, J)+C 1$

FU $(1, J 1)=F U \quad(1, J)+C 2$

FU $\quad(I I, J 1)=F U \quad(I I, J 1)+C 3$

$\mathrm{C}$

$\vdash U(I, J)=F U(I, J)+C 4$

HIX $=(P I J X * R U X(I,)-1.0.) / 2.0 *$ SIGNX

$f 1 Y=(1,0-R U Y(I, J) * 11,0-P I J Y)\} / 2,0 * S I G A Y$

$+2 X=(1.0-R \cup X(1 . J) *(1.0-P(J X)) / 2.0 * S(G N X$

$c$

F $2 Y=(P I J Y * R U Y(1, j)-1.0) / 2.0 * S I G A Y$

$F X U \quad(I 1, J)=F X U \quad([1, J)+F(X \quad * C 1$

FYU $(11, J)=F Y U$ (II,J)+FIY $* C 1$

FXU $(I, J 1)=F X U \quad(1, J I J+F 2 X \quad C 2$

$F Y U(I, J l)=F Y U(1, J I)+F 2 Y * C 2$

FXU $(I l, J 1)=F X(\quad(I I, J 1)+F 1 X+C 3$

$F Y U(I 1, J 1)=F Y U(I 1, J 1)+F 2 Y * C^{3}$

$F X U(1, J)=F X U(I, J)+F 2 X * C_{4}$

C

FYU $(I, J)=F Y U(I, J)+F I Y * C_{4}$

46.1

$46 \cdot 2$

46.3

46.4

46.5

46.6

46.7

46.8

47.1

47.2

47.3

47.4

47.5

47.6

47.7

47.8

48.1

48.2

48.3

48.4

48.5

48.6

48.7

48. 8

49.1

49.2

49.3

49.4

49.5

49.6

49.7

49.8

50.1

50.2

50.3

50.4

SC. 5

50.6

50.7

50.8

51.1

51.2

51.3

51.4

51.5

51.6

51.7

51.8

52.1

52.2

52.3

52.4

52.5

52.6

52.7

52.8

53.1

53.2

53.3

53.4

53.5

53.6

53.7

53.8

54.1

54.2

54.3

54.4

54.5

54.6

54.7

54.8 


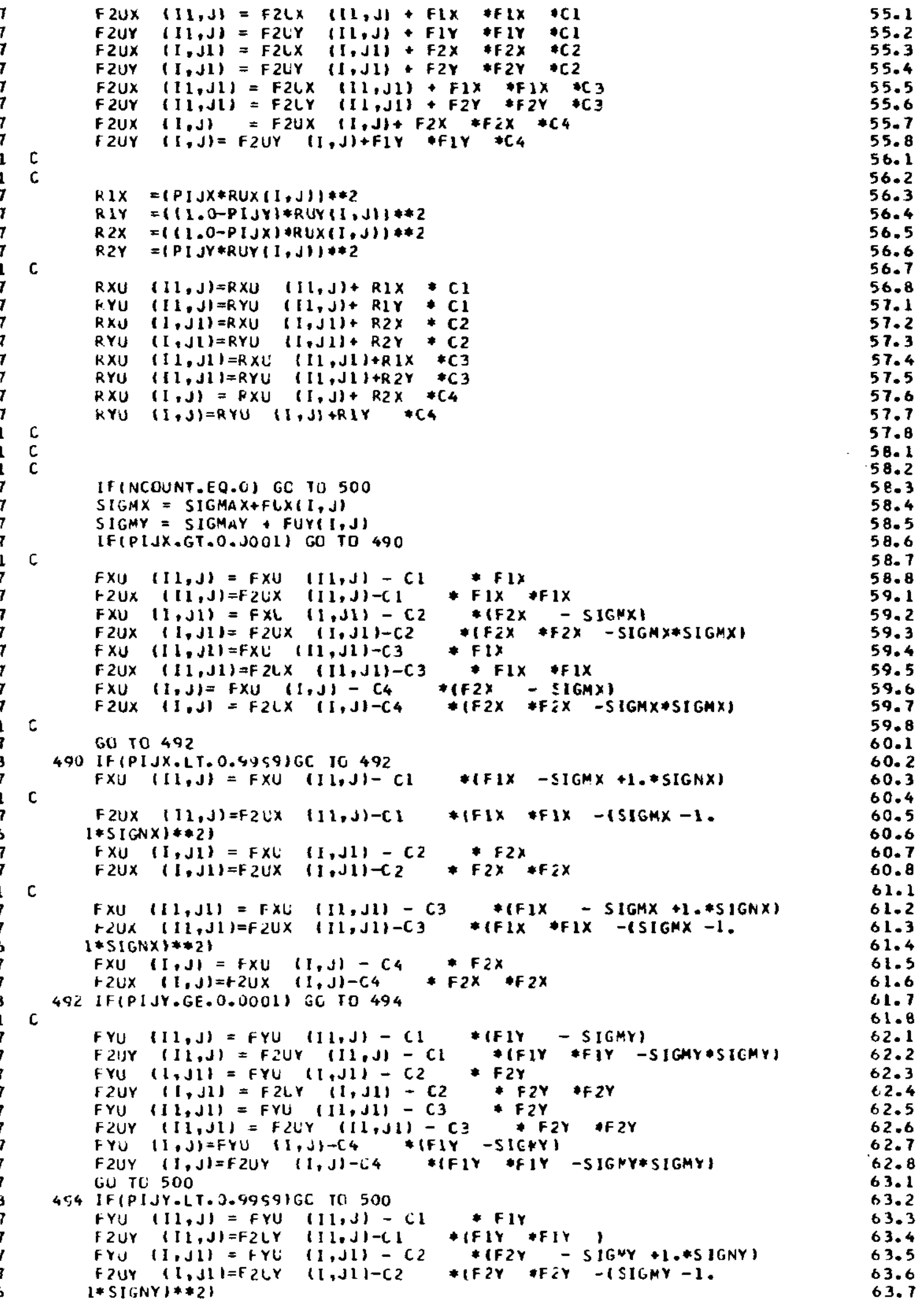




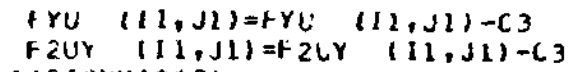
$1 * S I$ (SNY I $* 21$

FYU $(1, J)=$ FYU $(1, J)-C_{4}$ FIY

F 2 UY $(1, j)=F 2 U Y$ Y $(j, J)-C_{4}$ * Fir *Fir

5CO CGNTINUE

c

SCI CONTINUE

C

COUPLING BETHEEN ECRDER AND EULK

DC $351 I=1,1 \mathrm{~N}$

DU $550 \mathrm{~J}=1, \mathrm{JM}$

IF (I.EO.1.OR.1.EG.IN) GO TO 557

IF(J.EO.I.OR.J.EO.JW) GO TO 558

GU TO 550

557 IF(J.EO.1.OR.J.EC.JF) GO TO 550

SIGMAX $=V X \quad \forall T$ TI $\quad J X$

SIGMAY = VY *CT JOY

IFISIGMAX.EQ.0.) GC TC 552

SIGNX = SIGMAXIABS(SI GMAX)

GO 10554

552 SIGNX = 1 .

554 IF (SIGMAY.EQ.0, ) GC TO 553

SIGNY = SIGMAYIABS(SIGMAY)

GG TC 556

553 SIGNY $=1$.

$55611=I+l$

$\mathrm{d} 1=\mathrm{J}+1$

IF (SIGMAX.LT.0.) I I = I-

IFISIGMAY.LT.0.) JI $=\mathrm{J}-1$

IF(IL.LE-1.OR.II.GE.IN) GO TC 550 GO TC 559

558 IFII.LE.I.OR.I.CE.IN) GO TO 550

SIGMAX $=V X \quad \# T I O X$

SIGMAY = VY $\quad$ LT /DY

IF(SIGMAX.EQ.0.) GC TC 5520

SIGNX = SIGMAX/AES $(S I G$ MAX)

GO TO 5540

5520 SIGNX $=1$.

5540 IFISIGMAY.EO.O.) GC TC 5530

SIGNY = SIGMAY/AES(SIGMAY)

GO TO 5560

5530 SIGNY $=1$.

$5560 \begin{aligned} \text { Il } & =1 \\ J 1 & =J+1\end{aligned}+1$

If ISIGMAX .LI. O. III $=1-1$

IFISIGMAY.LT.0.) J1= J-l

IF(J1.LE.1.OR.J1.GE.JM) GC TC $55 \mathrm{C}$

$559 \mathrm{C} 1=C U(I, J) * A B S(S \mid G M A X) *(1,-A E S(S I G M A Y))$

$(2=C U(1, J) * A B S(5)$ GMAY $) *(1,-A E S(S I C M A X))$

$C 3=C U(I, J) * A B S(S(G N A X) * A B S(S I G M A Y)$

$c$

$(4=C U(I, J) *(I$. -AES $(S I G M A X)) *(1 .-A E S(S I G M A Y))$

FU $(11, J)=F U(11, J)+C 1$

$F U(I, J 1)=F U(i, J 1)+C 2$

$F U(11, J 1)=F U(1,, J 1)+C 3$

C

FU $(I, J)=F U(1, J)+C_{4}$

\begin{abstract}
$F X U(11, J)=F X L(11, J)+($ SIGMAX/2.-0.505ICNX)*C1
FYU $(11, J)=F Y U(11, J)+($ SIGMAY $/ 2, j * C 1$

$F X U(I, J 1)=F X U(1, J 1)+(S I G M A X / 2) * C$,

FYU $(1, J 1)=$ FYU $(1, J 1)+(S 1 G M A Y / 2 .-0.5 * 51 G N Y) * C 2$

FXU $(I 1, J I)=F X U(I 1 . J 1)+($ SIGMAX $/ 2 .-C .5 * 51 G N X) * C 3$

FYU $(11, J 1)=F Y L(11, J 1)+(51 G P A Y / 2 .-C .5 * S 1 G N Y) * C 3$

FXU $(1, J)=$ FXU $(1, J)+(51 \mathrm{GMAX} / 2) *.(4$

FYU $(1, J)=$ FYU $(1, J)+(51$ GMAY/2, $) * C 4$
\end{abstract}

c

F zUX $(11, j)=F 2(x$

FZUY $(11, J)=F 2 U Y$

F $2 U X$

$(J, J 1)=F 2 L x$

$(11, J)+(\operatorname{SIgMAX} / 2,-\mathrm{C}, 5 * \operatorname{SIGNX}) * 2 * \mathrm{C} 1$

$(I 1, J)+(S I G M A Y * 2 / 4) * C$,

$f \angle U Y$

$(1, J 1)=F 2 U Y$

$(I, J 1)+(S I G H A X * 2 / 4, \ldots) * C 2$

F $20 X$

$(1, J 1)+\left(S I G M A Y / 2 *-C_{0}\right.$ S*SIGNY $) * 2 * C 2$

$(11, j 1)+(S I G M A X) 2 .-C .5 * S I G N X) * 2 * C 3$

63.8

64.1

64.2

64.3

64.4

64.5

64.6

64.7

64.8

65.1

65.2

65.3

65.4

65.5

65.6

65.7

65.8

66.1

66.2

66.3

66.4

66.5

66.6

66.7

66.8

67.1

67.2

67.3

67.4

67.5

67.6

67.7

67.8

68.1

68.2

68.3

68.4

$6 B .5$

68.6

60.7

68.8

69.1

69.2

69.3

69.4

69.5

69.6

69.7

69.8

70.1

70.2

10.3

70.4

70.5

70.6

70.7

70.8

71.1

71.2

71.3

71.4

71.5

71.6

71.7

71.8

72.1

72.2

72.3

72.4

72.5

72.6

72.7

72.8 


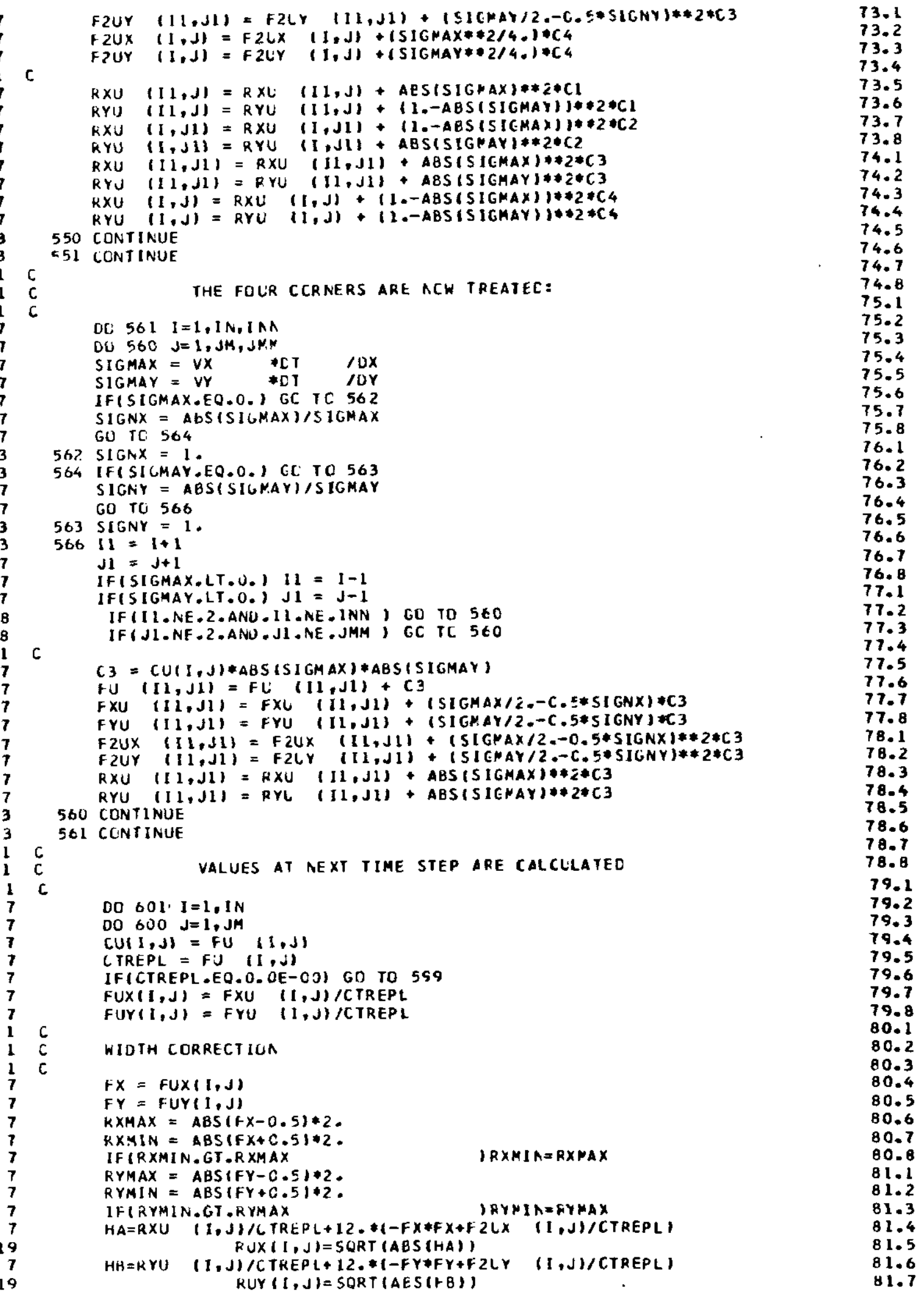




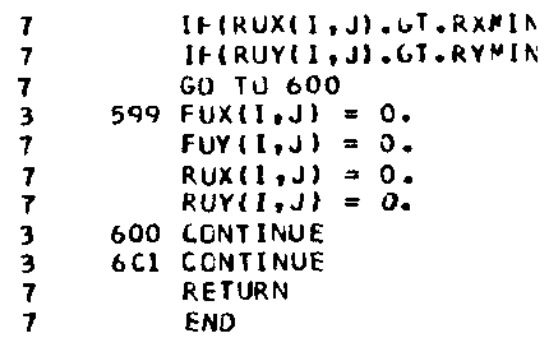

IRUX (I, J) R RXM IN

81.8 IRLY $(I, J)=R Y M I N$

82.2

82.3

82.4

82.5

82.6

82.1

82.8

83.1

83.2 


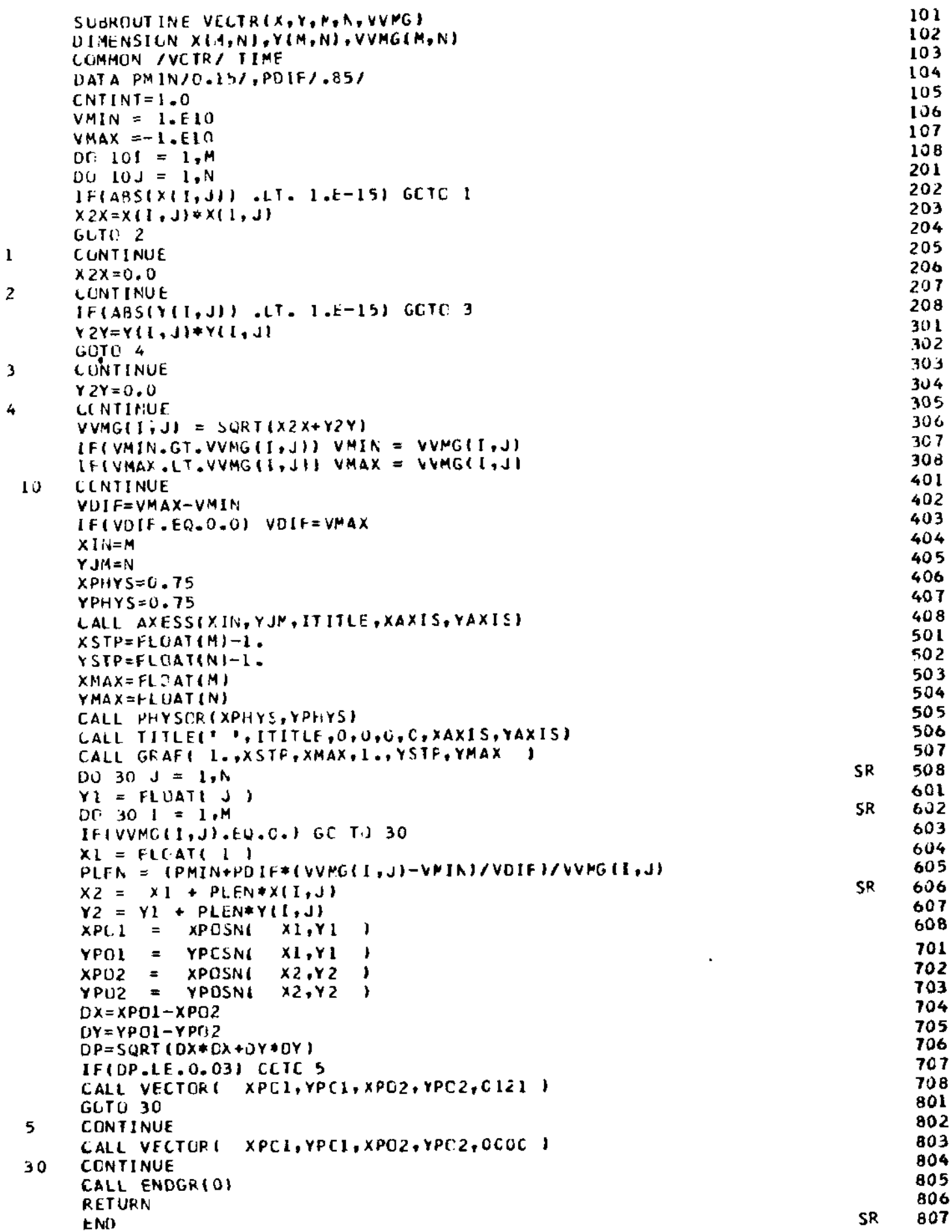


SUBROUTINE RANCET $(X, Y, N, N, I Y)$

1.1

$X I N=\mu$

$Y J M=N$

XPHYS $=0.75$

YPHYS $=0.75$

CALL AXESS (XIN,YJF,ITITLE,XAXIS, YAXIS)

XSTP = FLOAT $(M)-1$.

YSTP $=F \operatorname{LOAT}(N)-1$.

$X M A X=F \operatorname{LAT}(M)$

YMAX $=$ FLCAT IN)

CALL PHYSORIXPHYS, YPHYS

CALL TITLEI" "ITITLE, O, O,C,C,XAXIS,YAXISI

CALL GRAF ( $1 \ldots X S T P, X M A X, 1$, YSTP, YMAX)

DU $1 I=1,30$

DX: RANDU (IY)

DYI RANDU (IY)

$X I=x+D X$

$Y J=Y+D Y$

CALL HEIGMT $(0.04)$

CALL RLMESS $1 . .1, X I, Y J)$

1

CONT INUE

CALL ENOGR(O)

RETURN

END

1.2

1.3

$1 \cdot 4$

1.5

1.6

1.7

1. 8

2. 1

2.2

2.3

2.4

$2 \cdot 5$

2.6

2.7

2.8

3.1

3. 2

3. 3

3.4

3.5

3.6

3.7

3.8 


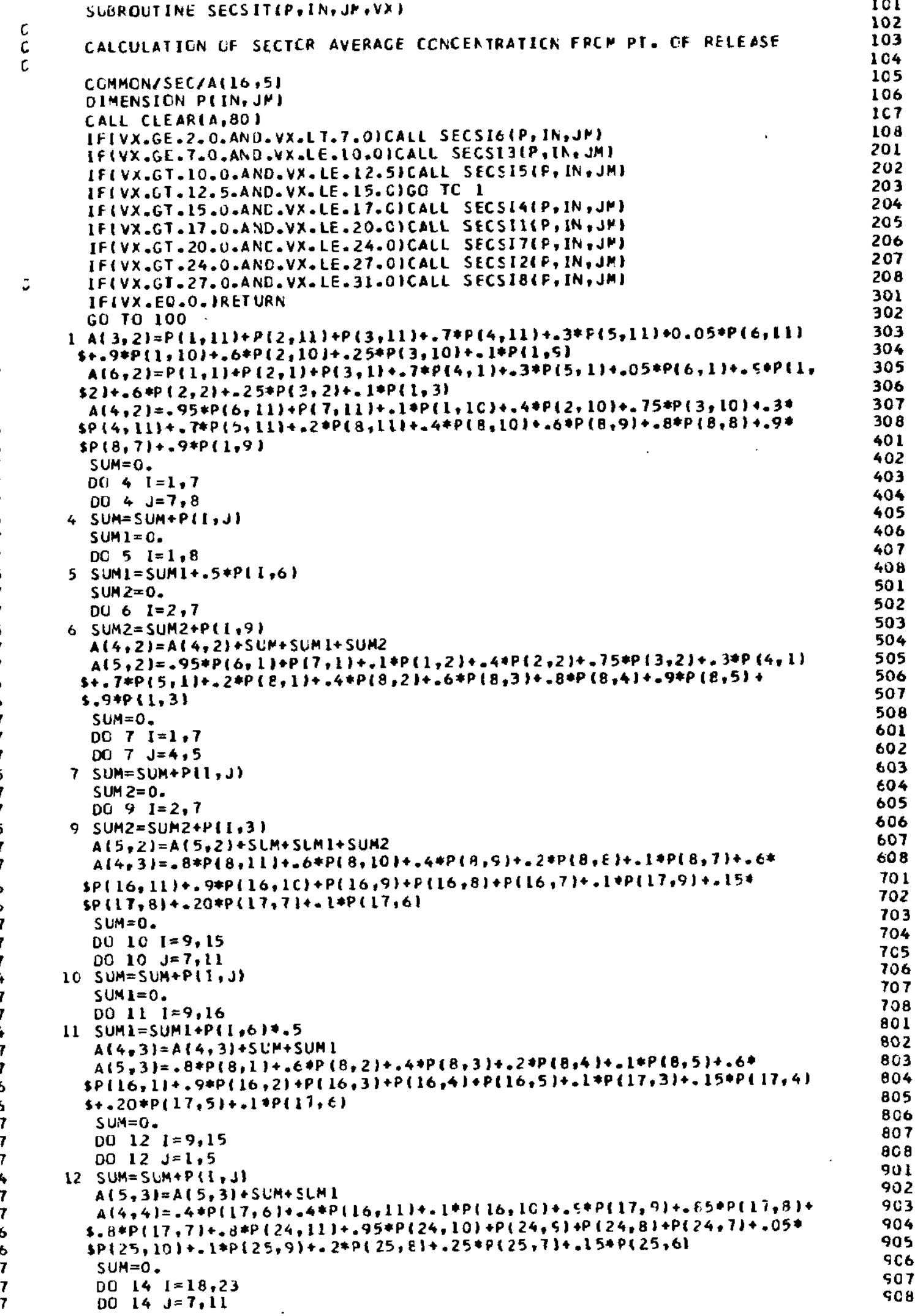


$14 S U M=S U M+P(1, J)$ SUM $1=\mathrm{C}$.

DO $15 \mathrm{I}=18.24$

15 SUM $1=\operatorname{SUM} 1+.5 * P(1,6)$

$A(4,4)=A(4,4)+S(M+S L M 1$

$A(5,4)=.4 * P(17,6)+.4 * P(16,1)+.1 * P(16,2)+., \$ P(17,3)+. E 5 * P(17,4)+$

S. $8 * P(17,5)+.8 * P(24,1)+.95 * P(24,2)+P(24,3)+P(24,4)+P(24,5)+. C 5 *$

$S P(25,2)+.1 * P(25,3)+.2 * P(25,4) * .25 * P(25,5)+.15 * P(25, \epsilon)$

SUM $=0$.

D0 $161=18,23$

DO $16 \quad J=1,5$

$16 S U M=S U M+P(I, d)$

$A(5,4)=A(5,4)+S(M+S L M 1$

$A(4,5)=.35 * P(25, E)+.2 * P(24,11) * .05 * P(24,10) * .55 * P(25,10) * .5 * P(25 *$

$54)+.8 * P(25,8)+.75 * F(25,7)$

SUM $=0$.

DU $17 I=26,30$

DO $i 7 \quad j=7,11$

17 SUM $=S U M+P(1, J)$

$S \cup M I=0$.

DO $18 \quad I=26,30$

18 SUM $I=S U M I+, 5 * P(I, C)$

$A(4,5)=A(4,5)+5 U A+S U A 1$

$A(5,5)=.35 * P(25,6)+.2 * P(24,2)+.05 * P(24,2)+.95 * P(25,2)+.9 * P(2 *, 3)$

$s+. A * P(25,4)+.75 * f(25,5)$

$S U M=0$.

DC $19 \quad I=26 \cdot 30$

DC $19 \mathrm{~J}=1,5$

$19 \quad S U M=S U M+P(1, J)$

$A(5,5)=A(5,5)+S U P+S(M)$

1 CO PRINT 105, VX

PRINT 104

PRINT $101,(1, I=1,16)$

DO $41 J=1.5$

41 WRITE(6, 102) (A) I,J),I =1,16)

(Cl FORMAT(IX,16(16,2X))

102 FORMAT $(2 x, 1611$ PEE.1)

C $0042 \mathrm{~J}=\mathrm{I}, 5$

[ 42 WRITE(7, 203$)(A(I, J), I=1,8)$

C DO $43 \mathrm{~J}=1.5$

C 43 WRITE $(7,103)(A(I, J), I=9,16)$

103 FORMAT(8) IPE 10.3))

104 FORMAT (IHO, 10X, 'SECTOR VALUES FRCM SITE IA 10 MI INCRENEATS",//I

105 FCRMAT $(1 M 0,5 X, "$ TRANSLATIONAL VELCCITY $=$,F5. $1, / 1)$ RETURN

END

SUBROUTINE SECS II (P,IA,JM)

COMMON/SEC/AI16,51

UIMENSION P(IN, JW)

$A(3,2)=.25 * P(1,21)+.05 * P(2,11)$

$A(6,2)=.25 * P(1,2)+.05 * P(2,1)$

$A(4,2)=.75 * P(1,11)+.95 * P(2,11)+P(3,11)+.45 * P(4,6)+.5 * P(4,7) * .8 *$

$S P(4,8)+.6 * P(4,9)+.45 * P(4,10)+.15 * P(4,11)$

SUM $=0$.

DD $20 \quad I=1,3$

DQ $20 \quad J=7,10$

20 SUM $=5 U M+P(1, J)$

SUM1 $=0$.

$0021 \quad I=1.3$

21 SUMI $=$ SUM $1 * P(1,6) * 5$

$A(4,2)=A(4,2)+S U M+S U M 1$

$A(5,2)=.75 * P(1,1)+.95 * P(2,1)+P(3,1)+.45 * P(4,6)+.9 * P(4,5)+.8 * F(4,4)$

$8+6 * P(4,3)+.45 * P(4,2) *-15 * P(4,1)$

$S \cup M=0$.

DO $22 I=1,3$

DO $22 \mathrm{~J}=2,5$

$22 S U M=S U M+P(I, J)$

$A(5,2)=A(5,2)+S(M M+S U M I$

$A(4,3)=.05 * P(4,6)+.1 * P(4,7)+.2 * P(4, Q)+.4 * P(4,9)+.55 * P(4,10)+.85 *$ $S P(4,11)+.7 * P(12,1)+.9 * P(12,10)+P(12,9)+P(12,8)+P(12,7)+.05 * F(13$, $\$ 91+.1 * P(13,8)+.15 * P(13,7)+.1 * P(13,6)$ $S U M=0$.

1102

1103

1104

1105

1106

1107

1108

1201

1202

1203

1204

1205

1206

1207

1208

1302

1302

1303

1304

1305

1306

1307

1308

1401

1402

1403

1404

1405

1406

$14 \mathrm{C} 7$

1408

1501

1502

1503

1504

1505

1506

1507

1508

1601

$16 \mathrm{C2}$

1603

1604

1605

1606

1607

1608

1701

1702

1703

1704

1705

1706

1767

1708

1801

1802

1803

1804

$18 \mathrm{CS}$

1806

1807

1808 
$D Q 23 \quad 1=5,11$

DO $23 \mathrm{~J}=7,11$

$23 S U M=S U M+P(I, J)$

SUMI $=0$.

DC $24 \quad I=5,12$

24 SU.1 $1=$ SUM $1+P(1,6) * .5$

$A(4,3)=A(4,3)+S U M+S C M)$

$A(5,3)=.05 * P(4,6)+.1 * P(4,5)+.2 * P(4,4) * .4 * F(4,3)+.55 * P(4,2)+. t 5 *$

$S P(4,1)+7 * P(12,1)+.9 * P(12,2)+P(12,3)+P(12,4)+P(12,5)+.05 * P(13,3)$

$5+.1 * P(13,4)+.15 * P(12,5)+.1 * P(13,6)$

SUM $=0$.

DC $25 \quad I=5,11$

DU $25 \mathrm{~J}=1,5$

$25 S U M=S U M+P(I, J)$

$A(5,3)=A(5,3)+S U N+\operatorname{SUM} 1$

$A(4,4)=.4 * P(13,6)+.55 * P(13,9)+.9 * P(13,8)+.85 * P(13,7)+.3 * P(12,11)$

\$* $=1 * P(12,10)+.85 * P(20,11)+55 * P(20,10)+C E * P(21,10)+P(20,9)+.1 *$

$S P(21,9)+P(20,8)+.1 E * P(21,8)+P(20,7)+.2 * P(2), 7)+.1 * P(21,6)$

$S U M=0$.

DU $26 \quad I=14,19$

$0026 \quad J=7,11$

$26 S U M=5 U M+P(1, J)$

SUM $1=0$.

$0027 \quad I=14,20$

27 SUM $1=\operatorname{SUM} 1+P(1,6) * 5$

$A\{4,4)=A(4,4)+$ SUM + SUM 1

$A(4,5)=.4 * P(13,6)+.55 * P(13,3)+.9 * P(13,4)+. E 5 * P(13,5)+.3 * P(12,1)$

$s+.1 * P(12,2) * .85 * P(20,1)+.05 * P(20,2)+.05 * P(21,2)+P(20,3)+.1 * P(21,3)$

$5+P(20,4)+.15 * P(21,4)+P(20,5)+.2 * P(21,5)+\ldots 1 * P(21,6)$

SUM $=0$.

DO $28 \quad 1=14,19$

DO $28 \quad J=1.5$

$28 S U M=S U M+P(I, J)$

$A(4,5)=A(4,5)+S(H+S L M 1$

$A(5,4)=.4 * P(21,6)+.15 * P(20,11)+.05 * P(2 C, 10)+.95 * P(21,10)+.9 * P(21,9$

$\$ 1+.85 * P(21,8)+.8 * P(21,7)+P(21,1)+.25 * P(29,6)+.5 * P(29,7)+.48 * P(29$,

$\$ 8)+.45 * P(25,9)+.4 C * P(29,10)+.35 * P(25,11)$

$S U Y=0$.

DC $29 \quad I=22,28$

DO $79 \mathrm{~J}=7,1 \mathrm{l}$

$29 S U M=5 U M+P(I, J)$

$\operatorname{SUM} 1=0$.

DO $30 \quad I=22,28$

30 SUM $1=S U M L+P(I, 6) * .5$

$A(5,4)=A(5,4)+5 L M+S \cup M 1$

$A(5,5)=.4 * P(21,6)+.15 * P(20,1)+.05 * P(20,2)+.55 * P(21,2)+.9 * P(21,3)$

$\$+.85 * P(21,4)+.8 * P(21,5)+P(21,1) * .25 * P(29,6)+.5 * P(29,5)+.48 * P(29,4)$

$5+.45 * P(29,3)+.40 * P(29,2)+.35 * P(29,1)$

SUM $=0$.

$0031 \quad I=22,28$

DO $31 \mathrm{~J}=1,5$

$31 S U M=S U M+P(I, J)$

$A(5,5)=A(5,5)+S U N+S U M 1$

RETURN

END

SUBROUT INE SECS I $2(P, 1 \mathrm{~A}, J M)$

COMHON/SEC/A(16,5)

DIMENSION P\{IN, IM\}

$A(4,2)=.45 * P(1,6)+. \varepsilon * P(1,7)+.7 * P(1,8)+.55 * P(1,9)+.4 * P(1,10)+.1 *$

$\operatorname{SP}(1,11)$

$A(5,2)=.45 * P(1,6)+.6 * P(1,5)+.7 * P(1,4)+.55 * P(1,3)+-4 * P(1,2)+.1 *$

$S P(1,1)$

$A(4,3)=.05 * P(1,6)+.2 * P(1,7)+.3 * P(1,8)+.45 * P(1,9)+.6 * P(1,10)+.9 *$

$5 P(1,11)+.1 * P(10,6) * .15 * P(10,7)+.1 * P(10,8) * .05 * P(10,9)+.95 * P(9,10$

s) $+.65 * P(9,11)+P(9, S)+P(9,8)+P(9,7)$

SUM $=0$.

$0032 \quad I=2,8$

DO $12 \mathrm{~J}=7,11$

$32 S U M=S U M+P(I, J)$

$\operatorname{SUM} 1=0$.

DO $33 I=2,9$ 
$33 S \cup M 1=S U M 1+P(I, 6) * 5$

$A(4,3)=A(4,3)+S(M+5 U M)$

$A(5,3)=.05 * P(1,6)+.2 * P(1,5)+.3 * P(1,4) * .45 * P(1,3)+.6 * P(1,2)+., S * P(1$,

$\$ 1)+.1 * P(10,6)+.15 * P(10,5)+.1 * P(10,4)+.05 * F(10,3)+.95 * P(9,2)+.65 *$

$S P(9,1)+P(9,3)+P(S, 4)+P(9,5)$

SUN $=0$.

DO $34 \quad 1=2,8$

$0034 \mathrm{~J}=1.5$

$34 S U M=S U M+P(I, J)$

$A(5,3)=A(5,3)+S L M+S L M 1$

$A(4,4)=.4 * P(10,6)+. \varepsilon 5 * P(10,7)+.5 * P(1 C, \varepsilon)+.55 * P(10,9)+.05 * P(9,10)$

$5+.45 * P(9,11)+P(17,7)+P(17,8)+P(17,9)+, 95 * F(17,10)+.75 * P(17,11)+$

$\$ .05 * P(18,9)+.1 * P(1 \varepsilon, 8)+\ldots 2 * P(16,7)+.1 * P(18,6)$

SU:A 20 .

DO $35 \quad 1=11,16$

DO $35 \mathrm{~J}=7,11$

35 SUM $=S U M+P(I, J)$

SUAl $=0$.

DO $36 \quad 1=11,17$

36 SUMl $=$ SUM $1+P(I .6) * .5$

$A(4,4)=A(4,4)+S U M+S L M 1$

$A(4,5)=.4 * P(10,6)+.85 * P(10,5)+.9 * P(10,4)+.55 * P(10,3)+0,0 \leqslant P(9,2)+$

$5.45 * P(9,1)+P(17,5)+P(17,4)+P(17,3)+.55 * P(17,2)+.75 * P(17,1)+.(5 *$

$\operatorname{sP}(18,3)+\ldots+P(18,4) * .2 * P(1, E, 5) * .1 * P(18,6)$

SUA $=0$.

$0037 \quad 1=11,16$

DC $37 \quad J=1,5$

$37 S U M=S U M+P(1, J)$

$A(4,5)=A(4,5)+S U M+S U M 1$

$A(5,4)=.4 * P(18,6)+.8 * P(19,7)+.9 * P(18,8)+.95 * P(18,9) * .05 * P(17,10)+$

S. $25 * P(17,11)+.5 * P(26,7)+.4 B * P(26, e)+.45 * P(26,9)+.40 * P(26,10)+.35$

$\operatorname{s*P}(26,1)+.25 * P(26,6)$

SUM $=0$.

DO $38 \quad I=19,25$

DU $38 \quad J=7,11$

$38 S U M=S U M+P(I, J)$

SUM $1=0$.

$0039 I=19,25$

39 SUM $I=S U M I+P(1,6) * .5$

$A(5,4)=A(5,4)+S(L N+\operatorname{SLN})$

$A(5,5)=.4 * P(18,6)+.8 * P(19.5)+.9 * P(18,4)+.95 * P(18,3)+.05 * P(17,2)+$

$5.25 * P(17,1)+.5 * P(26,5)+.48 * P(26,4)+.45 * F(26,3)+.40 * P(26,2) * .35 *$

$S P(26,1)+.25 * P(26, t)$

$S \cup M=0$.

DO $40 \quad I=19,25$

DU $40 \quad J=1.5$

$40 \quad S U M=S U M+P(I, J)$

$A(5,5)=A(5,5)+S U N+S L M 1$

RETURN

END

SUBROUT INE SECS $\{3(G, I N, J M)$

COMMON/SEC/AI 16,5

$c$

C IMENSION G(IN,JP), P(11,30)

$V X=8.04 \mathrm{M} / \mathrm{SEC}$

DC. $1 \quad I=1, I N$

00 i $J=1, j M$

I $P(J, I)=G(I, J)$

$A(2,1)=0.5 * P(11,1)$

$A(7,1)=0.5 * P(1,1)$

$A(3,1)=0,5 * P(1), 1)+.8 * P(11,2)+P(10,1)+P(10,2)+.6 * P(10,3)+P(5,1)$

$C+.9 * P(9,2)+.5 * P(9,3)+.33 * P(8,1)+.1 * P(8,2)$

$A(6,1)=0.5 * P(1,1)+.8 * P(1,2)+P(2,1)+F(2,2)+-6 * P(2,3)+P(3,1)+., * P(3$. C 2$)+.5 *(3,3)+.33 * P(4,1)+\ldots+1 * P(4,2)$

$A(4,1)=.07 * P(9,2)+. * * P(9,3)+.1 * P(9,4)+.6 * P(\varepsilon, 1)+.93 * P(8,2)+P(8,3)$ $C .3 * P(8,4)+P(7,1)+D(7,2)+P(7,3)+.5 * P(7,4)+.5 *(P(6,1)+P(6,2)+P(6,2))$ $C+.25 * P(6,4)$

$A(5,1)=.07 * P(3,2)+.5 * P(3,3)+.1 * P(3,4)+, \in * P(4,1)+.93 * P(4,2)+P(4,3) *$ $C \cdot 3 * P(4,4)+P(5,1)+P(5,2)+P(5,3)+.5 * P(5,4)+-5 *(P(6,1)+P(6,2)+P(6,3))$ $C+.25 * P(6,4)$

$A(3,2)=.12 * P(11,2)+.55 * P(11,3)+P(11,4)+P(1), 5)+P(11,6)+.9 * P(11,7)+$

C. $5 * P(11,8)+.1 * p(11,9)+.5 * P(10,3)+P(10,4)+.8 * P(10,5)+.3 * P(10, t)+.05$

2768

2801

2802

2803

2804

2805

2806

2807

2808

2901

2902

2903

2904

2905

2906

3907

2908

3001

3002

3003

3004

3005

3006

3007

3008

3101

3102

3103

3104

3105

3106

3107

3108

3201

3202

3203

3204

3205

3206

3207

3208

3301

3302

3303

3304

3305

3306

3307

3308

3401

3402

3403

3404

3405

3406

3407

3408

3501

3502

3503

3504

3505

3506

3507

3508

3601

3602

3633

3604

3605

3606

3607

3608 

$[, 8)+.1 * P(1,9)+.5 * P(2,3)+P(2,4)+.8 * P(2,5)+.3 * p(2,6)+. C 5 * 9(2,7)+.2 * P$ $C(3,4)$

$A(4,2)=-1 * P(11,7)+.5 * P(11,8)+. S * P(11,9)+P(11,10)+, 5 * P(11,11)+.2 * P($ $(10,5)+.7 * P(10,6)+.95 * P(10,7) * P(10,8)+P(10,5)+P(10, \quad 10) * .8 * P(10,11$ $C)+.8 * P(9,4)+P(9,5)+P(9,6)+P(9,7)+P(5,8)+P(9,9)+P(9,1()+P(9,11)+1 *$ $C P(9,12)+.7 * P(8,4)+P(8,5)+P(B, 6)+P(\varepsilon, 7)+P(\varepsilon, E)+P(8,9)+P(8,10)+P(E, 1$ $C(1+.2 * P(8,12)+.5 * P(7,4)+P(7,5)+P(7,6)+P(7,7)+P(7,8)+P(7,5)+P(7,10)$ $C+P(7,11)+.3 * P(7,12)+.2 * P(t, 4)+.5 *(P(6,5)+P(6,6)+P(6,7)+P(6,8)+P(6$, $(9)+P(6,10)+P(6,11))+2 * P(6,12)$

$A(5,2)=.1 * P(1,7)+.5 * P(1,8)+.9 * P(1,9)+P(1,10)+.5 * P(1,11) *, 2 * P(2,5)$ $C+.7 * P(2,6)+.95 * P(2,7)+P(2,8)+P(2,9)+P(2,1 C)+, B * P(2,11)+.8 * P(3,4) *$ $C P(3,5)+P(3,6)+P(3,7)+P(3,8)+P(3,9)+F(3,10)+P(3,11)+1 * P(3,12)+7 *$ $C P(4,4)+P(4,5)+P(4,6)+P(4,7)+P(4,8)+F(4,4)+P(4,10)+P(4,1)\}+2 * P(4,1$ $(2)+.5 * P(5,4)+P(5,5)+P(5,6)+P(5,7)+P(5,8)+P(5,9)+P(5,10)+P(5,11)+\ldots 3$ $C * P(5,12)+.2 * P(6,4)+.5 *(P(6,5)+P(6,6)+P(6,7)+P(t, 8)+F(6,9)+P(6,10)$ $C+P(6,11))+.2 * P(6,12)$

$A(4,3)=.5 \neq P(11,11)+P(1), 12)+P(11,13)+P(11,14)+P(11,151+P(11,16)$ $C+P(11,17)+P(11,1 E)+.9 * P(11,19)+, 2 * P(10,11)+P(10,12)+P(7,13)+P(7,14$ $C)+P(7,15)+P(7,16)+P(7,17)+P(7,18)+P(7,19)+-7 * P(7,20)+.3 * P(6,12)+5$ $C *(P(6,13)+P(6,14)+P(6,15)+P(6,16)+P(6,17)+P(6,18)+P(6,19))+.4 * P(6$, C 201

$A(5,3)=-5 * P(1,11)+P(1,12)+P(1,13)+P(1,14)+P(1,15)+P(1,16)+P(1,17)+$ C $P(1,18)+.9 * P(1,19)+.2 * P(2,11)+P(2,12)+P(2,13)+P(2,14)+P(2,15)+$ $C P(2,16)+P(2,17)+P(2,18)+P(2,19)+1, P(2,2 C)+.9 * P(3,12)+P(3,1)+$ C $P(3,14)+P(3,15)+P(3,16)+P(3,17\}+P(3,18)+P(3,19\}+.3 * P(3,20)+.8 * P(4$ $C, 12)+P(4,13)+P(4,14)+P(4,15)+P(4,16)+P(4,17)+P(4,18)+P(4,19)+5 *$ $C P(4,20)+\ldots * P(5,12)+P(5,13)+P(E, 14)+P(5,15)+P(5,16)+P(5,17)+P(5,18)$ $C+P(5,19)+.7 * P(5,2))+.3 * P(6,12)+.5 *(P(6,13)+P(6,14)+P(6,15)+P(6,16)$ $C+P(6,17)+P(6,18)+F(6,19))+, 4+P(6,20)$

$A(4,4)=-1 * P(1), 19)+P(11,20)+P(11,21)+P(11,22)+P(11,23)+P(11,24)+$ $C P(11,25)+P(11,26)+P(11,27)+.2 * P(11,28)+.9+P(10,20)+P(10,21)+P(1 C$, $C(22)+P(10,23)+P(1 C, 24)+P(10,25)+P(10,26)+P(10,27)+.4 * P(10,28)+.7 *$ $C P(9,20)+P(9,2)+P(9,22)+P(9,23)+P(9,24)+P(5,25)+P(9,26)+P(9,27)+$ $C .7 * P(9,28)+.5 * P(8,20)+P(8,2)+P(8,22)+P(8,23)+P(8,24)+P(8,25)+P(8$, $(26)+P(8,27)+.7 * P(8,28)+, 3 * P(7,20)+P(7,21)+P(7,22)+P(7,23)+P(7,24)+$ $C P(7,25)+P(7,26)+P(7,27)+.8 * P(7,28)+.1 * P(6,20)+.5 *(P(6,21)+P(6,22)+$ $C P(6,23)+P(6,24)+P(6,25)+P(6,26)+P(6,2))\}+4 * P(6,28)$

$A(5,4)=01 \neq P(1,19)+P(1,20)+P(1,21)+P(1,22)+P(1,23)+P(1,24)+P(1,25)+$ $C P(1,26)+P(1,27)+.2 * P(1,28)+.9 * P(2,20)+P(2,21)+P(2,22)+P(2,23)+P(2$. $C 24)+P(2,25)+P(2,26)+P(2,27)+.4 * P(2,28)+, 7 * P(3,20)+P(3,21)+P(2,22)+$ $C P(3,23)+P(3,24)+P(3,25)+P(3,26)+P(3,27)+.7 * P(3,28)+.5 * P\{4,20)+P\{4$, $C 21)+P(4,22)+P(4,23)+P(4,24)+P(4,25)+P(4,26)+P(4,27)+.7 * P(4,2 E)+.3 *$ $C P(5,20)+P(5,21)+P(5,22)+P(5,23)+P(5,24)+P(5,25)+P(5,26)+P(5,27)+.8$ $C * P(5,28)+.+P(6,20)+.5 *(P(6,2))+P(6,22) * P(6,23)+P(6,24)+P(6,25)+$ $C P(6,26)+P(6,27))+.4 * P(6,28)$

$A(4,5)=.8 * P(1), 28)+P(11,29)+P(1), 30)+.6 * P(10,28)+P(10,29)+P(10,30)$ $C+.3 * P(9,28)+P(9,29)+P(9,30)+.3 * P(8,28)+P(8,29)+P(8,30)+.2 * P(7,28)+$ $C P(7,29)+P(7,30)+.1+P(6,28)+.5 *(P(6,29)+P(6,30))$

$A(5,5)=.8 * P(1,28)+P(1,29)+P(1,30)+6 * P(2,28)+P(2,29)+P(2,3 C)+3 *$ $C P(3,28)+P(3,29)+P(3,30)+.3 * P(4,28)+P(4,29)+P(4,30)+.2 * P(5,28)+P(5$, $C 29)+P(5,30)+.1 * P(6,28)+.5 *(P(6,29)+P(t, 20))$

RE TURN

END

SUBROUTINE SECSI $14(G, I N, J M)$

COMMON/SEC/A(16,5)

DIMENSION G(IN,SM),P(11,30)

$V X=16.5 M / S E C$

UO I I $=1$, IN

DO $1 \mathrm{~J}=1, \mathrm{JM}$

l $P(J, I)=G(I, J)$

$A(3,2)=P(11,1)+.75 * P(11,2)+.25 * P(1), 3)+.15 * P(10,1)$

$A(6,2)=P(1,1)+.75 * P(1,2)+.25 * P(1,3) * .15 * P(2,1)$

$A(4,2)=.25 * P(1), 2)+.75 * P(1), 3)+P(1), 4)+.9 * P\{1), 5\}+.5 * P(10,1)+$ r. $P(10,2)+P(10,3)+P(10,4)+P(10,5)+.2 * P(10,6)+P(5,1)+P(9,2)+P(5,3)+$ $C P(9,4)+P(9,5)+.5 * P(5,6)+P(8,1)+P(8,2)+P(8,3)+P(8,4)+P(8,5)+\ldots 5 * P(B$ $C, 6)+P(7,1)+P(7,2)+P(7,3)+P(7,4)+P(7,5)+.8 * P(7, C)+5 *(P(6,1)+P(6,2)$ $C+P(6,3)+P(6,4)+P(6,5))+0,4+P(6,6)$

$A(5,2)=.25 * P(1,2)+.75 * P(1,3)+P(1,4)+.9 * P(1,5)+.85 * P(2,1)+P(2,2)+$

3702

3703
3704

3705

3706

3707

3708

3801

3802

3803

3804

3805

3806

3807

3808

3901

3902

3903

3904

3905

3906

3907

3908

4001

4002

4003

4004

4005

4006

4607

4008

4101

4102

4103

4104

4105

4106

4107

4108

4201

4202

4203

4204

4205

4206

4267

4208

4301

4302

4303

4304

4305

4206

4307

4308

4401

4402

4403

4404

4405

4406

4407

4408

4501

4502

4503

4504

4505

4506

4507

4508 
$C P(2,3)+P(2,4)+P(2,5)+. ? * P(2,6)+P(3,1)+P(3,2)+P(3,3)+P(3,4)+P(3,5)+$ $C .5 * P(3,6)+P(4,1)+P(4,2)+P(4,3)+P(4,4)+P(4,5)+.7 * P(4,6)+P(5,1)+P(5$, $C 2)+P(5,3)+P(5,4)+P(5,5)+.8 * P(5,6)+.5 *(P(E, 1)+P(t, 2)+P(6,3)+P(6,4)+$ $C P(6,5))+.4 * P(6,6)$

$A(4,3)=.1 * P(11,5)+P(1), 6)+P(11,7)+P(1), B)+P(11,9)+P(1), 10)+P(1), 11$ $(1+P(11,12)+P(11,13)+.3 * P(11,14)+.8 * P(10,6)+P(10,7)+P(10,8)+P(10,9)$ $C+P(10,10)+P(10,11)+P(10,12)+P(1 C, 13)+.6 * P(10,14)+.5 * P(9,6)+P(9,7)+$ $C P(9, B)+P(9,9)+P(9,10)+P(9,1)+P(9,12)+P(9,13)+.85 * P(9,14)+.25 * P(\theta$, $C 6)+P(8,7)+P(8,8)+P(\epsilon, 9)+P(8,10)+P(8,11)+P(8,12)+P(8,13)+P(8,14)+, 2$ $C \neq P(7,6)+P(7,7)+P(7,8)+P(7,9)+P(7,10)+P(7,11)+P(7,12)+P(7,13)+P(7,1$ $C 4)+.15 * P(7,15)+.1 * P(6,6)+.5 *(P(6,7)+P(6,8)+P(t, 5)+P(6,10)+P(6,11)+$ $C P(6,12)+P(6,13)+P(6,14))+1, P(6,15)$

$A(5,3)=.1 * P(1,5)+P(1,6)+P(1,7)+P(1,8)+P(1,9)+P(1,10)+P(1,11)+P(1,1$ $(2)+P(1,13)+.3 * P(1,14)+.8 * P(2,6)+P(2,7)+P(2,8)+P(2,5)+P(2,10)+P(2,1$ $C(1+P(2,12)+P(2,13)+.6 * P(2,14)+.5 * P(3,6)+P(3,7)+P(3,8)+P(3,9)+P(3,1$ $C 0)+P(3,11)+P(3,12)+P(3,13)+. E 5 * P(3,14)+.25 * P(4,6)+P(4,7)+P(4,8)+$ $C P(4,9)+P(4,10)+P(4,11)+P(4,12)+P(4,13)+P(4,14)+, 2 * P(5,6)+P(5,7)+$ $C P(5, B)+P(5,9)+P(5,10)+P(5,11)+P(5,12)+P(5,13)+P(5,14)+15 * P(5,15)+$ $C .1 * P(6,6)+.5 *(P(6,7)+P(6,8)+P(6,9)+P(6,10)+P(6,11)+P(6,12)+P(6,13)$ $C+P(6,14))+.1 * P(6,15)$

$A(4,4)=.7 * P(11,14)+P(11,15)+P(11,16)+P(11,17)+P(11,18)+P(11,19)+$ $C P(11,20)+P(11,2(1)+75 * P(1), 22)+, 4 * P(10,14)+P(10,15)+P(1 C, 16)+P(10$, $C 17)+P(10,18)+P(10,19)+P(1 C, 20)+P(1 C, 21)+, 5 * P(10,22)+.15 * P(9,14)+$ $C P(9,15)+P(9,16)+P(5,17)+P(9,18)+P(9,19)+P(9,20)+P(9,21)+P(9,22)+$ $C P(8,15)+P(8,16)+P(8,17)+P(8,1 E)+P(8,19)+P(8,20)+P(8,21)+P(8, Z 2)+$ $C .1 * P(8,23)+.85 * P(7,15)+P(7,16)+P(7,17)+P(7,18)+P(7,19)+P(7,20)+$ $C P(7,21)+P(7,22)+.2 * P(7,23)+.4 * P(6,15)+.5 *(P(6,16)+P(6,17)+P(6,18)+$ $C P(6,19)+P(6,20)+P(6,21)+P(6,22))+.1 * P(6,23)$

$A(5,4)=.7 * P(1,14)+P(1,15)+P(1,16)+P(1,17)+P(1,18)+P(1,19)+P(1,20)+$ $C P(1,21)+.75 * P(1,22)+.4 * P(2,14)+P(2,15)+P(2,16)+P(2,17)+P(2,16)+$ $C P(2,19)+P(2,20)+P(2,21)+.9 * P(2,22)+.15 * P(3,14)+P(3,15)+P(3,16)+$ $C P(3,1)+P(3,18)+P(3,19)+P(3,20)+P(3,21)+P(2,22)+P(4,15)+P(4,16)+$ $C P(4,17)+P(4,18)+P(4,19)+P(4,20)+P(4,21)+P(4,22)+, 1+P(4,23)+.85 *$ $C P(5,15)+P(5,16)+P(5,17)+P(5,18)+P(5,19)+P(5,20)+P(5,21)+P(5,22)+, 2$ $C * P(5,23)+.4 * P(6,1 \leq)+.5 *(P(6,16)+P(6,17)+P(6,1 \varepsilon)+P(6,19)+P(6,2 C)+$ $C P(6,2)+P(6,22))+(1 * P(6,23)$

$A(4,5)=.25 * P(11,22)+P(1), 23)+P(1), 24)+P(1,1,25)+P(1), 26)+P(1), 27)+$ $C P(1,28)+P(11,29)+P(11,30)+11 * P(10,22)+P(10,23)+P(10,24)+P(1 C, 25)+$ $C P(10,26)+P(10,27)+P(10,28)+P(10,29)+P(1 C, \geq 0)+P(9,23)+P(9,24)+P(9,2$ $C 5)+P(9,26)+P(9,27)+P(9,28)+P(9,29)+P(9,30)+.9 * P(8,23)+P(8,24)+$ $C P(8,25)+P(\theta, 26)+P(8,27)+P(8,28)+P(8,29)+P(8,3 C)+8 * P(7,23)+P(7,24)$ $C+P(7,25)+P(7,26)+P(7,27)+P(7,28)+P(7,29)+P(7,20)+.4 * P(6,23)+.5 * 1$ $C P(6,24)+P(6,25)+P(6,26)+P(6,27)+P(6,28)+P(6,25)+P(6,30))$

$A(5,5)=.25 * P(1,22)+P(1,23)+P(1,24)+P(1,25)+P(1,26)+P(1,27)+P(1,28)$ $C+P(1,29)+P(1,30)+.1 * P(2,22)+P(2,23)+P(2,24)+P(2,25)+P(2,26)+F(2,2)$ C) $+P(2,28)+P(2,29)+P(2,30)+P(3,23)+P(3,24)+P(3,25)+P(3,26)+P(3,27)+$ $C P(3,28)+P(3,29)+P(3,30)+.9 * P(4,23)+P(4,24)+P(4,25)+P(4,26)+P(4,27)$ $C+P(4,28)+P(4,29)+P(4,30)+.8 * P(5,23)+P(5,24)+P(5,25)+P(5,26)+P(5,27$ $C)+P(5,28)+P(5,29)+P(5,30)+.4 * P(6,23)+.5 *(F(E, 24)+P(6,25)+P(6,26)+$ $C P(6,27)+P(6,28)+P(6,29)+P(6,3 C))$

RETURN

END

SUBROUTINE SECSISIG,IN, JM)

CUMMON/SEC/A(16,5)

DIMENSION G(IN, $\{M), P(11,30)$

VELOCITY IS $11.6 \mathrm{C} / \mathrm{SEC}$

DO $1 I=1, I N$

DO $1 \mathrm{~J}=1, \mathrm{JM}$

l $P(J, t)=G(1, J)$

$A(3,1)=-1 * P(10,1)+.25 * P(9,1)$

$A(6,1)=.1 * P(2,1)+.25 * P(3,1)$

$A(4,1)=.3 * P(9,1)+.5 * P(8,1)+P(7,1)+.5 * P(6,1)$

$A(5,1)=.3 * P(3,1)+. S \otimes P(4,1)+P(5,1)+.5 * P(6,1)$

$A(3,2)=P(1), 1)+P(1), 2)+P(11,3)+P(1), 4)+.75 * P(11,5)+.25 * P(1),()+.9 *$

$C P(10,1)+.9 * P(10,2)+.6 * P(10,3)+.2 * P(10,4) * .2 * P(9,1)+\ldots 1 * P(9,2)$

$A(6,2)=P(1,1)+P(1,2)+P(1,3)+P(1,4)+.75 * P(1,5)+.25 * P(1,6)+.9 * P(2,1)$ $C+.9 * P(2,2)+.6 * P(2,3)+.2 * P(2,4)+.2 * P(3,1)+.1 * P(3,2)$

$A(4,2)=.25 * P(11,5)+.75 * P(11,6)+P(11,7)+P(11,8) * .9+.1 * P(10,2)+.4 *$ $C P(10,3)+.8 * P(10,4)+P(10,5)+P(10,6)+P(10,7)+P(10,8)+.2 * P(10,9)+.25 *$ $C P(9,1)+.9 * P(9,2)+P(9,3)+P(9,4)+P(5,5)+P(9,6)+P(9,7)+P(9,8)+, 6 * P(9)$

4601

4602

4603

4604

4605

4606

4607

4608

4701

4702

4703

4704

4705

4706

4707

4708

4801

4802

4803

4804

4805

4806

4807

4808

4901

4902

4903

4904

4905

4906

4907

4908

5001

5002

5003

5004

5005

5006

5007

5008

5101

5102

5103

5104

5105

5106

5107

5108

5201

5202

5203

5204

5205

5206

5207

5208

5301

5302

5303

5304

5305

5306

5307

5308

5401

5402

5403

5404

5405

5406

5407

5408 
$(9)+.1 * P(8,1)+P(8,2)+P(9,3)+P(E, 4)+F(8,5)+P(8,6)+P(8,7)+P(B, E)+, 8 *$ $C P(3,9)+P(7,2)+P(7,3)+P\{7,4\}+P(7,5)+P(7,6)+P(7,7)+P(7,8)+9 * P(7,9)+$ $C .5 *(P(6,2)+P(6,3)+P(6,4)+P(6,5)+P(6,6)+P(t, 7)+P(6,8)+P(6,9))$

$A(5,2)=.25 * P(1,5)+.75 * P(1,6)+P(1,7) * .5 * F(1,8)+.1 * P(2,2)+.4 * P(2,3)+$ $C . B * P(2,4)+P(2,5)+P(2, t)+P(2,7)+P(2, f) * 2 * P(2,9)+2,5 * P(3,1)+5 * P(3$, $(2)+P(3,3)+P(3,4)+P(3,5)+P(3, t)+P(3,7)+P(3, E)+.6 * P(3,9)+\ldots 1 * P(4,1)+$ $C P(4,2)+P(4,3)+P(4,4)+P(4,5)+P(4,6)+P(4,7)+P(4,8)+, 8 * P(4,9)+P(5,2)+$ $C P(5,3)+P(5,4)+P(5,5)+P(5,0)+P(5,7)+P(5, E)+, C * P(5,9)+0,5 *(P(6,2)+P(6$ $C, 3)+P(6,4)+P(6,5)+F(6,6)+P(6,7)+P(6,8)+P(6,4))$

$A(4,3)=.1 * P(11,8)+.95 \neq P(11,9)+P(1), 10)+P(11,12)+P(11,13)+P(11,14)+$ $C P(1), 15)+P(11,16)+.4 * P(11,17)+.7 * P(1 C, 9)+P(10,1 C)+P(10,11)+P(10,12$ ()$+P(10,13)+P(10,14)+P(10,15)+P(10,16)+, 7 * P(10,17)+.4 * P(5,9)+P(9,10$ $(1)+P(9,11)+P(9,12)+P(9,13)+P(5,14)+P(S, 15)+P(9,16)+.85 * P(9,17)+15 *$ $C P(8,9)+P(8,10)+P(9,11)+P(8,12)+P(8,12)+P(8,14)+P(8,15)+P(8,16)+P(8$ $C, 17)+.1 * P(7,9)+P(7,10)+P(7,11)+P(7,12)+P(7,13)+P(7,14)+P(7,15)+P(7$ $C, 16)+P(7,17)+.1+P(7,18)+5 *(P(6,10)+P(6,11)+P(6,12)+P(0,1,3)+P(6,14$ $(1+P(6,15)+P(6,16)+f(6,17))+\ldots+P(6,18)$

$A(5,3)=.1 * P(1,8)+S E * P(1,9)+P(1,10)+P(1,11)+P(1,12)+P(1,13)+F(1,14$ $C)+P(1,151+P(1,161+, 4 * P(1,17)+.7 * P(2,9)+P(\leqslant, 10)+P(2,11)+P(2,12)+$ $C P(2,13)+P(2,14)+P(2,15)+P(2,16)+.7 * P(2,1)+.4 * P(3,9)+P(3,10)+P(3,1$ $C(1) P(3,12)+P(3,12)+P(3,14)+P(3,15)+P(3,16)+.85 * P(3,17)+.15 * P(4,9)+$ $C P(4,10)+P(4,1)\}+P(4,12)+P(4,13)+P(4,\{4)+P(4,15)+P(4,16)+P(4,17)+.1$ $C * P(5,9)+P(5,10)+P(5,11)+P(5,12)+P(5,13)+P(5,14)+P(5,15)+P(5,16)+$ $C P(5,17)+.1 * P(5,18)+.5 *(P(6,10)+P(6,11)+P(6,12)+P(6,13)+P(6,14)+P(6$ $(, 15)+P(6,15)+P(6,17))+1 * P(6,18)$

$A(4,4)=.6 * P(11,17)+P(11,18)+P(1), 19)+P(11,20)+P(11,21)+P(11,22)+$ $C P(11,23)+P(11,24)+.8 * P(1,25)+.3 * P(10,17)+P(1 C, 18)+P(10,19)+P(10,2$ $C 0)+P(10,21)+P(10,22)+P(1), 23)+P(10,24)+.95 * P(10,25)+.15 * P(9,17)+$ $C P(9,18)+P(9,19)+P(S, 20)+P(9,2)+P(S, 22)+P(9,23)+P(9,24)+P(S, 25)+$ $C .05 * P(9,26)+P(8,18)+P(B, 19)+P(8,20)+P(8,21)+P(8,22)+P(8,23)+F(8,24$ $(1+P(8,25)+.15 * P(8,26)+.9 * P(7,18)+F(7,19)+F(7,20)+P(7,21\}+P(7,22\}+$ $C P(7,23)+P(7,24)+P(7,25)+.2 * P(7,26)+.4 * P(t, 18)+.5 *(P(6,19)+P(6,20)+$ $(P(5,2)+P(6,22)+P(6,23)+P(6,24)+P(6,25))+P(t, 26) * .1$

$A(5,4)=.6 * P(1,17)+F(1,18)+P(1,15)+P(1,20)+P(1,2)+P(1,22)+P(1,23)+$ $C P(1,24)+. B * P(1,25)+.3 \neq P(2,17)+P(2,18)+P(2,19)+P(2,2 C)+P(2,21)+P(2$, $622)+P(2,23)+P(2,24)+.95 * P(2,25)+.15 * P(3,17)+P(3,18)+P(3,19)+P(3,20$ $C)+P(3,21)+D(3,22)+P(3,2))+P(3,24)+P(3,25)+C(5 * P(3,26)+P(4,18)+P(4$, $C(9)+P(4,26)+P(4, Z 1)+P(4,22)+P(4,23)+P(4,24)+P(4,25)++15 * P(4,26)+$ $C .4 * P(5,18)+P(5,191+P(5,20)+P(5,21)+P(5,22)+F(5,23)+P(5,24)+P(5,25)$ $C+P(5,26) * .2+.4 * P(6,18)+.5 *(P(t, 15)+P(t, 20)+P(t, 21)+P(t, 22)+P(6,23)$ $(+P(0,24)+P(6,25))+1 * P(6,26)$

$A(4,5)=-2 * P(1), 251+P\{1\}, 2 \in\}+P(1), 27)+f(1), 28)+P(11,29)+P(1), 30)+$ $C .05 * P(10,25)+P(1 C, 26)+P(10,27)+P(10,28)+P(10,29)+P(10,3 C)+.5 \leqslant * P(9$, $(26)+P(9,27)+P(9,28)+P(7,29)+P(5,30)+.85 * P(8,26)+P(8,27)+P(8,28)+$ $C P(8,29)+P(8,10)+.8 * P(7,26)+P(7,27)+P(7,28)+P(7,29)+P(7,30)+, 4 * P(6$, $(26)+.5 *(P(6,27)+P(t, 28)+P(6,29)+P(6,30))$

$A(5,5)=.2 * P(1,25)+P(1,26)+P(1,27)+P(1,2 q)+P(1,25)+P(1,30)+C E * P(2$, $(25)+P(2,26)+P(2,27)+P(2,28)+P(2,29)+P(2,30)+.55 * P(3,26)+P(3,27)+$ $C P(3,28)+P(3,29)+P(3,3 C)+.85 * P(4,26)+P(4,2)+P(4,28)+P(4,29)+F(4,30$ $C)+.8 * P(5,26)+P(5,27)+P(5,2 B)+P(5,29)+P(5,30) *-4 * P(6,26)+.5 *(P(6,27$ $C)+P(6,28)+P(0,29)+P(6,30))$

RET JRN

END

SUBROUTINE SECS $16(\mathrm{C}, I \mathrm{~N}, \mathrm{JM})$

CGMMON/SEC/A(16,5)

DIMENSIGI G(IN,JN), P( 11,30$)$

VELCCITY IS $4.0 \mathrm{~N} N$ /SEC

20 $1 I=1, I N$

DU $1 J=1, J M$

l $P(J, I)=G(1, j)$

$A(2,1)=P(11,1)+P(1), 2)+.5 * P(1), 3)+P(10,1)+.5 * P(10,2)+, 5 * P(0,1)$

$A(7,1)=P(1,1)+P(1,2)+.5 * P(1,3)+P(2,1)+-5 * P(2,2)+.5 * P(3,1)$

$A(3,1)=.5 * P(1), 3)+.8 B * P(1), 4)+.12 * P(11,5)+.5 * P(10,2)+P(10,4)+.6 *$ $C P(10,5)+.5 * P(9,1)+P(9,2)+P(9,3)+.94 * P(9,4)+-6 * P(9,5)+P(8,1)+.75 *$ $C P(8,2)+.4 * P(8,3)+.1 * P(8,4)+\ldots 3 * P(7,1)$

$A(6,1)=.5 * P(1,3)+.8 E * P(1,4)+.12 * P(1,5)+.5 * P(2,2)+P(2,3)+P(2,4)+.6 *$ $C P(2,5)+.5 * P(3,1)+P(3,2)+P(3,3)+.94 * F(3,4)+.6 * P(3,5)+P(4,1)+.75 *$

$C P(4,2)+.4 * P(4,3)+.1 * P(4,4)+.3 * P(5,1)$

$A(4,1)=.1 * P(9,4)+.4 * P(9,5)+.2 * P(9,6) * .25 * P(8,2)+.6 * P(8,3)+.9 * P(8,4$ $C)+P(5,5)+.35 * P(\varepsilon, 6)+, 15 * P(7,1)+P(7,2)+P(7,3)+P(7,4)+P(7,5)+, 6 * P(7$,

5501

5502

5503

5504

5505

5500

5507

5508

5001

5602

5603

5004

5605

5606

5607

5608

5701

5702

$57 \mathrm{C} 3$

5704

5705

5706

5707

5708

5801

5802

5803

5804

5805

$58 \mathrm{Co}$

$580 ?$

5800

5901

5902

5903

5904

5905

5906

5907

5908

6001

6002

6003

6004

6005

6006

6007

6008

6101

6102

6103

6104

6105

6106

6107

6108

6201

6202

6203

6204

6205

6206

6207

6208

6301

6302

6303

6304

6305

6306

6307

6308 
CP $(6,3)$

$A(5,2)=P(1,1)+, 4 * P(1,2)+P(2,1)+.75 * P(2,2)+P(3,1)+P(3,2)+P(4,1)+P(4$ $=, 2)+.2 * P(4,3)+P(5,1)+P(5,2)+.25 * P(5,3)+.5 *(P(t, 1)+P(6,2))+.2 * P(6,3$ c)

$A(4,3)=.6 * P(11,2)+F(1,1,3)+P(11,4)+P(11,5)+P(1,1,6)+P(11,7)+P(11, P)+$ $C P(1,, 9)+P(11,10) * 5+.25 * P(1 C, 2)+P(1 C, 3)+P(10,4)+P(10,5)+P(10,6) *$ $C P(10,7)+P(10,8)+P(10,9)+P(10,10)+\ldots \cup P(16,1)+P(5,3)+P(9,4)+P(9,5)+$ $C P(9,6)+P(9,7)+P(5, E)+P(9,9)+P(9,10)+.3 * P(9,11)+.8 * P(8,3)+P(8,4)+$ $C P(8,5)+P(8,6)+P(6,7)+P(8,8)+P(8, S)+P(8,10)+.5 * P(8,1)+\ldots, 75 * P(7,3) *$ $C P(7,4)+P(7,5)+P(7,6)+P(7,7)+P(7,8)+P(7,9)+P(7,10)+.65 * P(7,1)+\ldots * 3 *$ $C P(6,3)+.5 *(P(6,4)+P(6,5)+P(6,6)+P(6,7)+P(6,9)+P(6,10))+3 * P(t, 11)$

$A(5,3)=.6 * P(1,2)+P(1,3)+P(1,4)+P(1,5)+P(1,6)+P(1,7)+P(1,8)+P(1,5)+$ C. $9 * P(1,10)+.25 * P(2,2)+P(2,3)+P(2,4)+F(2,5)+P(2,6)+P(2,7)+P(2,8) 4$ $C P(2,9)+P(2,10)+\ldots+P(2,11)+P(3,3)+P(3,4)+P(3,5)+P(3,()+P(3,7)+P(3,8$ C $)+P(3,9\}+P\{3,10\}+.3 * P(3,11)+Q * P(4,3)+P(4,4)+P(4,5\}+P(4,6)+P(4,7\}+$ $C P(4, y)+P(4,9)+P(4,10)+.5 * P(4,11)+.75 * P(5,3)+P(5,4)+P(5,5)+P(5,6)+$ $C P(5,7)+P(5, B)+P(5,9)+P(5,10)+.65 * P(5,11)+.3 * P(6,3)+.5 *(P(6,4)+P(6$, $(5)+P(6,6)+P(6,7)+P(6,9)+P(6,9)+P(6,10))+\ldots * P(6,11)$

$A(4,4)=-1 \neq P(11,10)+P(11,11)+P(11,12)+P(11,13)+P(11,14)+F(11,15)+$ $C P(11,16)+P(1), 17)+P(1), 18)+.2 * P(11,19)+.9 * P(10,11)+P(10,12)+F(1 C, 1$ $C 31+P(10,14)+P(10,15)+P(1), 16)+P(10,17)+P(10,18)+.4 * P(10,19)+.7 *$ $C P(9,11)+P(9,12)+P(5,12)+P(9,14)+P(9,15)+P(9,16)+P(9,17)+P(9,19)+.6$ $C * P(9,19)+.5+P(8,1)+P\{8,12\}+P(8,13\}+P(B, 14)+P(8,15\}+P(8,16)+P(8,17$ $C)+P(8,18)+.7 * P(8,15)+.35 * P(7,11)+P(7,12)+F(7,13)+P(7,14)+P(7,15)+$ $C P(7,16)+P(7,17)+P(7,18)+.8 * P(7,19)+.2 * P(6,11)+.5 *(P(6,12)+P(6,13)+$ $C P(6,14)+P(6,15)+P(t, 16)+P(6,1)\}+P(6,18)\}+.1+P(6,19)$

$A(5,4)=.1 \neq P(1,10)+P(1,11)+P(1,12)+P(1,13)+P(1,14)+P(1,15)+P(1,16)+$ $C P(1,17)+P(1,18)+P(1,19)+2+.9+P(2,11)+P(2,12)+P(2,13)+P(2,14)+P(2$, $[15)+P(2,16)+P(2,1]\}+P(2,18)+04+P(2,19)+, 7+P(3,1)\}+P(3,12)+P(3,13)+$ $C P(3,14)+P(3,15)+P(3,16)+P(3,17)+P(3,18)+, C * P(3,19)+, 5 * P(4,1))+P(4$, $C(2)+P(4,13)+P(4,14)+P(4,15)+P(4,16)+P(4,17)+P(4,18)+, 7 * P(4,15)+.35$ $C * P(5,11)+P(5,12)+P(5,13)+P(5,14)+P(5,15)+F(5,16)+P(5,17)+P(5,18)+$ C. $6 * P(5,19)+.2 * P(6,11)+.5 *(P(6,12)+P(6,13)+P(6,14)+P(6,15)+P(6,16)+$ $C P(6,17)+P(6,18))+\ldots 1 * P(6,19)$

$A(4,5)=.8 * P(11,19)+P(11,20)+P(11,21)+P(11,22)+P(1), 23)+F(1), 24)+$ $C P(11,25)+P(11,26)+.8 * P(11,27)+.8 * P(10,19)+P(1 C, 20)+P(10,21)+F(1 C, 2$ $C 2)+P(10,23)+P(10,24)+P(10,25)+P(10,26)+0,5 * F(10,27)+4 * P(9,19)+$ $C P(9,2 C 1+P(9,21)+P(9,22)+P(9,23)+P(9,24)+P(9,25)+P(9,26)+.9 * F(9,27)$ $C+.3 * P(8,19)+P(8,20)+P(8,21)+P(8,22)+P(8,23)+P(8,26)+P(8,25)+F(8,26$ $(1+.95 * P(8,27)+.2 * P(7,19)+P(7,20)+P(7,21)+P(7,22)+P(7,23)+P(7,24)+$ $C P(7,25)+P(7,26)+.95 * P(7,27)+.4 * P(6,19)+.5 *(P(t, 20)+P(6,21)+P(t, 22)$ $C+P(6,23)+P(6,24)+P(6,25)+P(6,26)+P(6,27))$

$A(5,5)=.8 * P(1,19)+P(1,20)+P(1,21)+P(1,22)+P(1,23)+P(1,24)+P(1,25)+$ $C P(1,26)+.8 * P(1,27)+.6 * P(2,19)+P(2,2 C)+P(2,21)+P(2,22)+P(2,23)+P(2$, $(24)+P(2,25)+P(2,26)+., 85 * P(2,27)+, 4 * P(3,19)+P(3,20)+P(3,2)+P(3,22)$ $C+P(3,23)+P(3,24)+P(3,25)+P(3, z t)+. S * P(3,27)+, 3 * P(4,19)+P(4,2()+$ $C P(4,21)+P(4,22)+P(4,23)+P(4,24)+P(4,25)+P(4,26)+.95 * P(4,27)+.2 *$ $C P(5,19)+P(5,20)+P(5,21)+P(5,22)+P(5,23)+P(E, 24)+P(5,25)+P(5,26)+$ $(.45 * P(5,27)+.4 * P(6,19)+.5 *(P(t, 20)+F(t, 21)+P(t, 22)+P(6,23)+P(6,24)$ $C+P(6,25)+P(6,26)+P(6,27))$

KETURN

FND

SUBKOUTJNE SECS I $8(P, I A, J M)$

C C.1 IIN/SEC/A(16,5)

UI YENSICN P(IN, JM)

$A(4,3)=P(1,11)+P(Z, 11)+P(3,11)+P(4,11)+P(5,11)+, 3 * P(6,11)+P(1,10)+$ $* P(2,10)+P(3,1(1)+P(4,10)+P(5,10)+.5 * P(6,10)+P(1,5)+P(2,9)+P(3,5)+$

$* P(4,9)+P(5,9)+E * F(6,5)+P(1,8)+P(2,8)+P(3,8)+P(4, B)+P(5, B)+$

$* .9 * P(6,8)+P(1,7)+P(2,7)+P(3,7)+P(4,7)+P(5,7)+P(6,7)+1 * P(7,7)+$

$* .5 * P(1,6)+.5 * P(2,6)+.5 * P(3,6)+.5 * P(4,6)+. \leqslant * P(5,6)+.5 * P(6,6)+$

$* 1 * 0(7,6)$

$A(5,3)=.5 * P(1,6)+.5 * P(2,6)+-5 * P(3,6)+.5 * P(4,6)+-5 * P(5,6)+.5 * P(0,6)$

*+. $1 * P(7,6)+P(1,5)+P(2,5)+P(3,5)+P(4,5)+P(5,5)+P(6,5)+1 * P\{1,5\}+$

$* P(1,4)+P(2,4)+P(2,4)+P(4,4)+P(5,4)+, G * P(6,4)+P(1,3)+P(2,3)+P(3,3)$.

$* P(4,3)+P(5,3)+.8 * P(6,3)+P(1,2)+P(2,2)+P(3,2)+P(4,2)+P(5,2)+$

* $5 * P(6,2)+P(1,1)+P(2,1)+P\{3,1)+P\{4,1\}+P(5,1)+.3 * P(6,1\}$

$A(4,4)=.7 * P(6,11)+P(7,11)+P(\varepsilon, 11)+P(5,11)+P(1 C, 11)+P(1), 11)+$

$* P(12,11)+P(13,11)+.7 * P(14,11)+.5 * P(t, 10)+P(7,1 C)+P(E, 10)+P(9,1 C)+$

$* P(10,10)+P(1), 1 C\}+P(12,10)+P(13,10)+8 * P(14,10\}+, 2 * F(6,9)+P(7,9\}+$

$* P(8,9)+P(C, 9)+P(10,3)+P(11,9)+P(12,5)+P(12,5)+P(14,5)+1 * P(t, 8)+$

$* P(1,8)+P(8,8)+P(9,8)+P(1), 8)+P(1,8)+P(12,8)+P(13,8)+P(14,8)$ *

*. $\{* P(15,8)+.9 * P(7,7)+P(8,7)+P(9,7)+F(10,7)+F(1), 7)+P(12,7)+P(13,7)$

7403

7404

7405

7406

7407

7408

7501

7502

7503

7504

7505

7506

7507

7508

7601

7602

7603

7604

7605

7606

7607

7608

7701

7702

7703

7704

7715

7706

7707

7708

7801

7802

7803

7804

7805

78 J6

7807

7808

7901

7902

7963

7964

79.15

7966

7907

7908

8ิง

8col

A003

$80 \div 4$

80105

Bou6

8007

\&) 8

8101

8112

9103

$81 \times 4$

3105

4106

8107

8108 


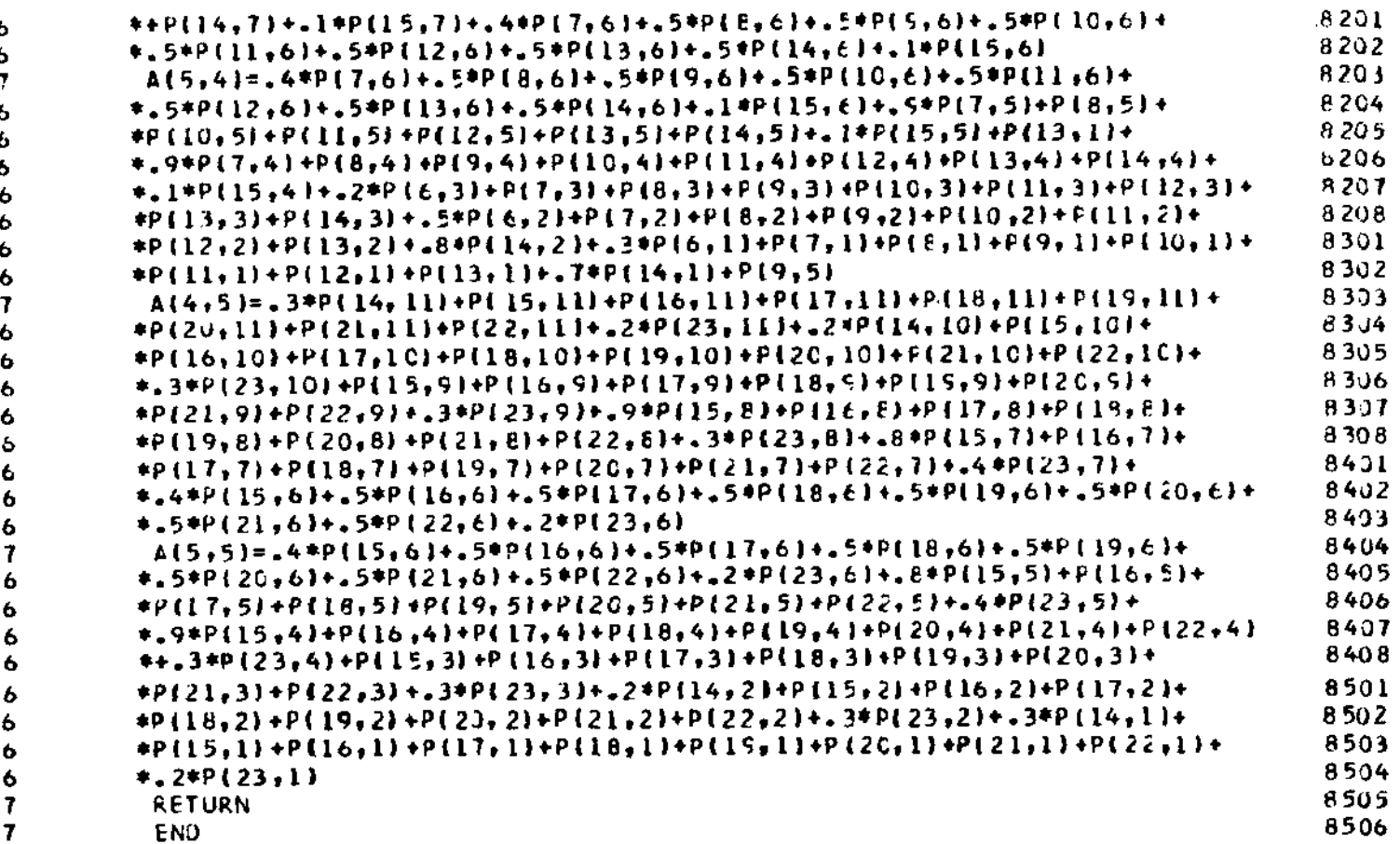




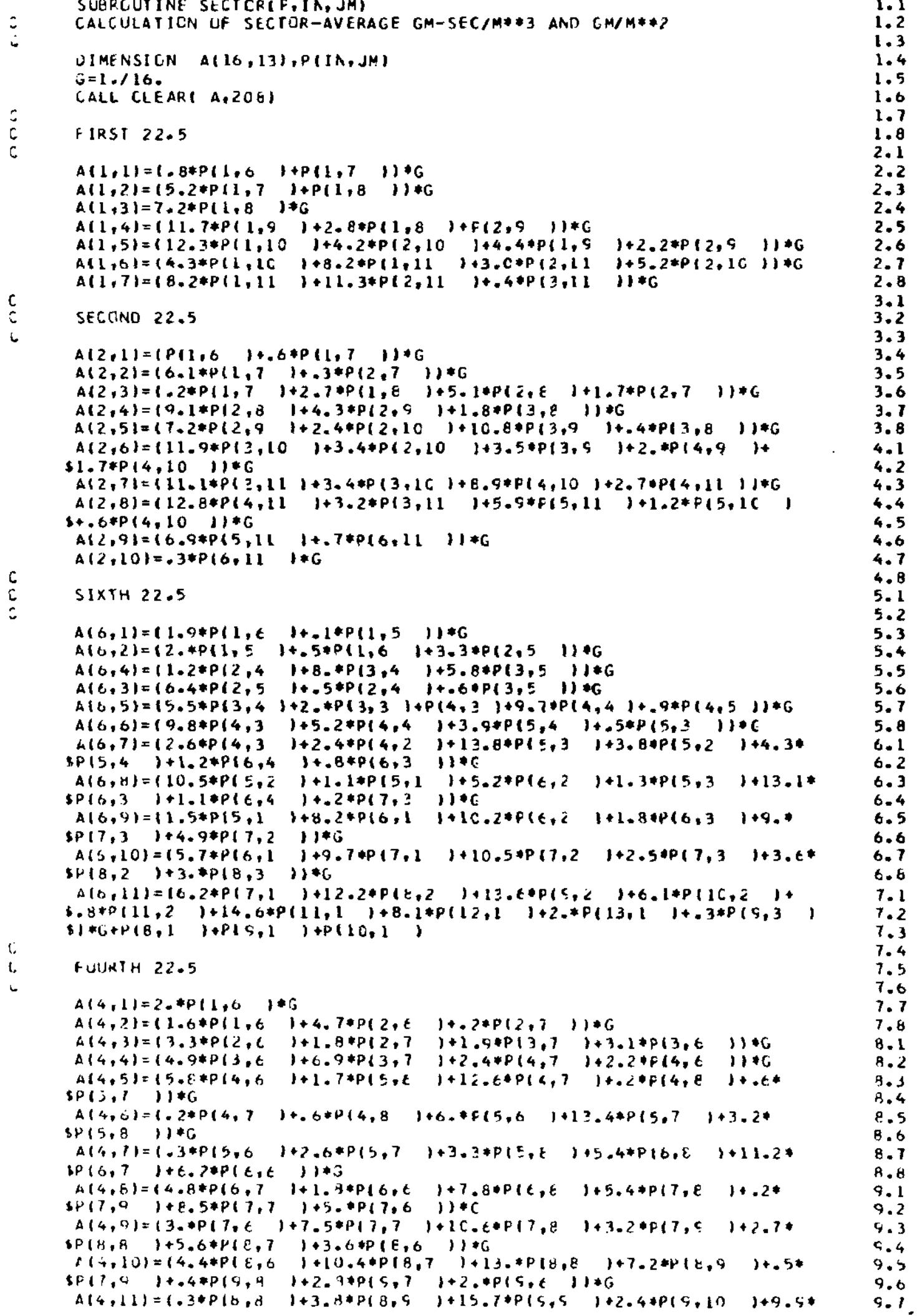




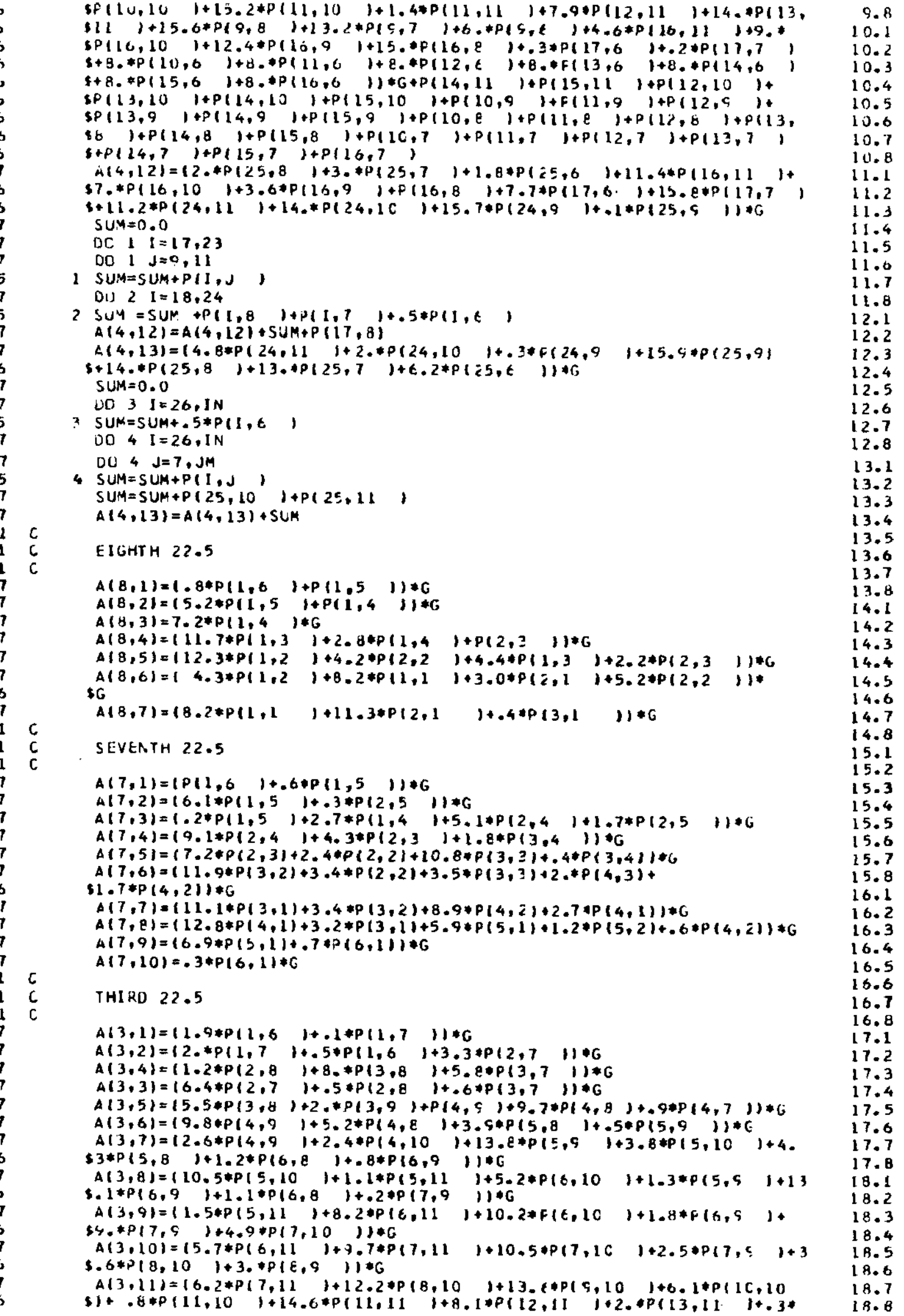


$\$ P(9,0,1) * G+P(8,11,1+P(9,1),+P(1), 1)\}$

19.1

19.2

FIFTH 22.5

$A(5,1)=2 . * P(1,0) * E$

$A(5,2)=(1,6 * P(1, t)+4.7 * P(2, t \quad 1+.2 * P(2,5)) * 6$

$A(5,3)=13.3 * P(2, C)+1.0 * P(2,5$

$A(5,4)=\{4.9 * P(3,6 \quad 1+6.9 * P(3,5$

$A(5,5)=15.8 * P(4,6 \quad 1+1.7 * P(5,6$

$1+1.9 * P(3.5 \quad 1+3.1 * P(3,6 \quad 1) * G$

$1+2.4 * F(4,5 \quad 1+2.2 * P(4,6) 1) * 6$

$\operatorname{sp}(5,5) *$,

$A(5,6)=(.2 * P(4,5) \quad+.6 * P(4,4)+6 . * P(5,6) 1+13.4 * P(5,5 \quad)+7.2 *$

$S P(5,4,1) * G$

$A(5,7)=1.3 * P(5,6 \quad)+2.6 * P(5,5 \quad 1 * 3.3 * P(5,4 \quad 1+5.4 * P(6,4 \quad 1+11.2 *$

$S P(S, 5)+6.2 * P(\epsilon, 6)) * G$

$A(5,8)=(4.8 * P(6,5)+1 . B * P(6,6 \quad)+7.8 * P(6,4 \quad 1+5.4 * P(7,4 \quad 1+.2 *$

SP $\{7,3 \quad 1+8.5 * P 17,5 \quad 1+5 . P 17,6 \quad 11 * G$

$A(5,9)=13 . * P(7, t)+7.5 * P(7,5 \quad 1+10.6 * P(7,4 \quad 1+3.2 * P(7,3 \quad 1+2.7 *$

P $(3,4),+5.6 * P(8,5,1+3.6 * P(8,6) 1) * G$

$A(5,10)=(4.4 * P(\varepsilon, 6) \quad 1+1\}, 4 * P(8,5 \quad 1+13 . * P(8,4 \quad 1+7.2 * P(8,3 \quad 1+.5 *$

SP $(7,3) 1+.4 * P(9,4) 1+2.8 * P(9,5) 1+2 . * P(9,6) 1) * G$

$A(5,11)=1.3 * P(8,4 \quad 1+3.8 * P(8,3) 1+15.7 * P(5,3) \quad+2.4 * P(5,2) 1+9.5 *$

P $(10,2) 1+15.2 * P(1), 2)+1.4 * P(1), 1)+7.9 * F(12,1) 1+14 . * P(13,1)$

$\$ P(10,2 * P(9,4)+13,2 * P(9,5)+6 .+P(5,6) 1+4.6 * P(16,1) 1+5$.

$S P(10,2,1+12,4 * P(16,3)+15, * P(16,4)+\ldots 3 * P(17,6) 1+.2 * P(17,5)$,

$5+8 . * P(10,6)+8 . * P(11,6)+8 . * P(12, t)+Q . * P(13,6)+8 * * P(14,6)$

$S P(13,2),+P(14,2)+P(15,2)+P(10,3)+P(11,3) 1+P(12,3)+$

$\$ P(1\}, 3 \quad 1+P(14,3) \quad 1+P(15,3 \quad 1+P(10,4))+P(1), 4) \quad+P(12,4) 1+P(13$,

$54)+P(14,4)+P(15,4),+P(10,5))+P(11,5)+P(12,5)+P(13,5)$

$5+P(14,5) \quad+P(15,5)+P(10,5)$

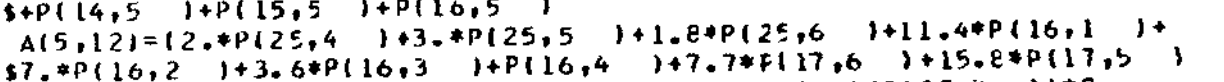

$6 * P(16,3)+P(16,4,7 * P(24,3)+.1 * P(25,3)) \neq C \quad 23.1$

SU: $1=0.0$

DO $5 \quad I=17,23$

Di) $5 \mathrm{~J}=1,3$

5 SUM $=S U M+P(I, J)$

DO $6 \quad I=18,24$

6 SU.A $=$ SUM $+P(1,4 \quad)+P(1,5 \quad)+.5 * P(1, t \quad)$

$A(5,12)=A(5,12)+S U M+P(17,4)$

$A(5,13)=14.8 * P(24,1,1+2, * P\{24,2,1+, 3 * P(24,3$

$1+15.9 * P(25,3)$

19.3

19.5

19.6

19.7

19.0

20.1

20.2

20.3

20.4

20.5

20.6

26.7

20.8

21.1

21.2

21.3

21.4

21.5

21.6

21.7

21.8

22.1

22.2

22.3

22.4

22.5

22.6

23.2

23.3

23.4

23.5

23.6

23.7

23.8

24.1

24.2

24.3 SUM $=0.0$

DO $7 \quad 1=26, I N$

7 SUY $=$ SUM+. $5 * P(1,6)$

DU $B \quad I=26,1 A$

DC $8 \quad J=1,5$

$8 \quad S U M=S U M+P(I, J)$

$S U M=S U M+P(25,2 \quad 1+P(25,1)$

$A(5,13)=A(5,13)+S U M$

PRINT SECTOR AVERAGES

PRINT $1 \cup 0,(1, I=1,16)$

DO $\rightarrow J=1,13$

Q WRI TE $(6,101)(A(I, 4), I=1,16)$

10U FORMATIIX,16(16,2XI)

101 FOR MAT $(2 x, 16(1 P E E=1)$

C DO LO J $=1,13$

C Ii WRITE $(7,102)(A)[, J), I=1,8)$

nO $11 \mathrm{~J}=1,13$

c. 11 WRITE(7,102)(AI [,J), 1 =9,16)

102 IJRRAT (8) (LPEIO.3)

RETURN

ENO

24.4

24.5

24.6

24.7

24.8

25.1

25.2

25.3

25.4

25.3

25.6

25.7

25.8

26.1

26.2

26.3

26.4

26.5

26.6

26.7

26.8

27.1 


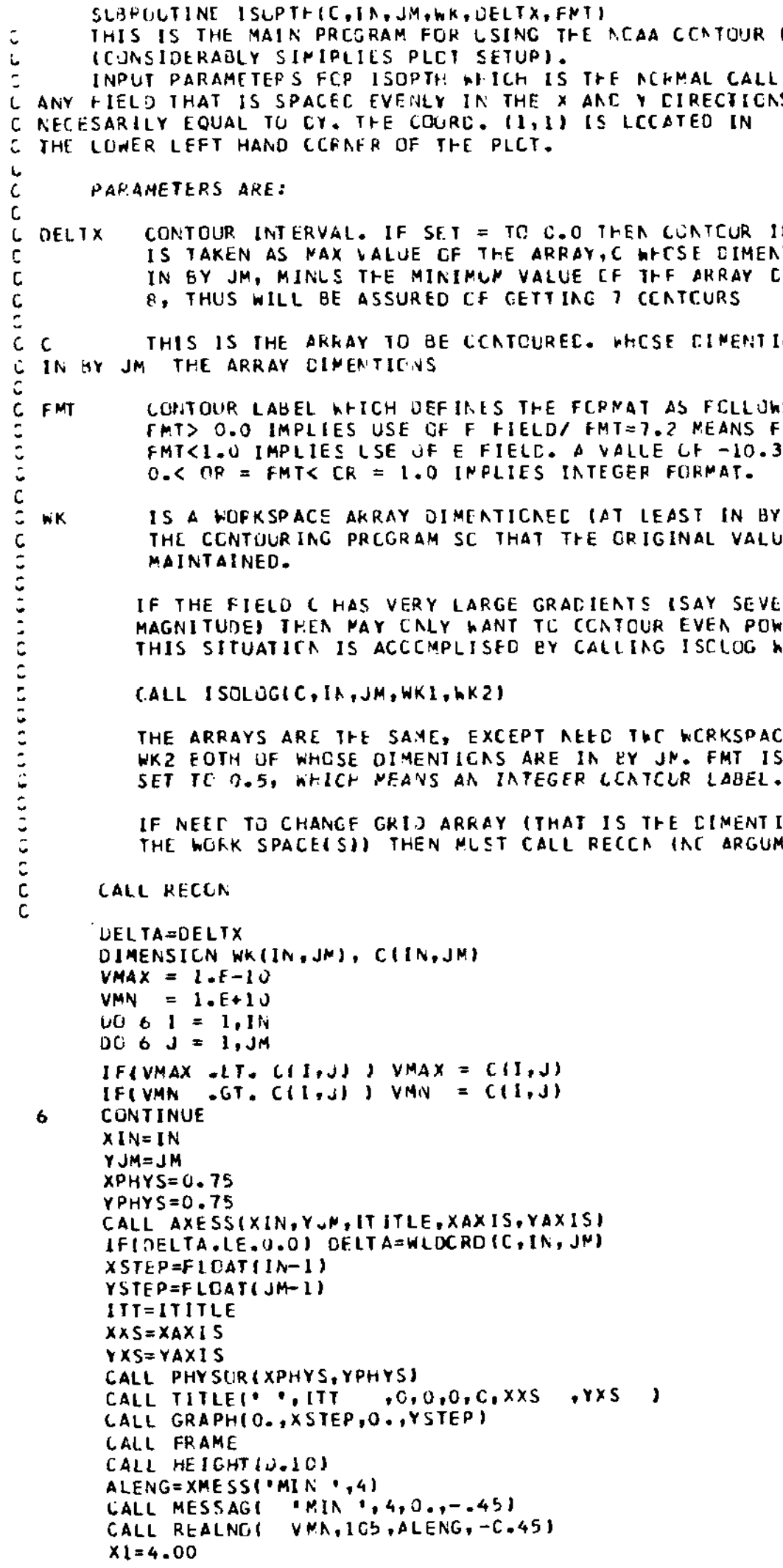

(.ALL ISOLUGIC, IA,JM,WKI,WK2) SET TC 0.5, NHICH NEANS AN IATEGER CCATCLR LABEL.

CALL RECCN

IF THE FIELO C HAS VERY LARGE GRACIEATS ISAY SEVERAL CRUERS DF MAGNITUDE) JHEA MAY CALY WANT TC CCATOUR EVEA POWERS CF TEN THIS SITUATICA IS ACCCMPLISED EY CALLIAG ISCLOG WHOSE CALL IS

THE ARRAYS ARE THE SAYE, EXCEPT AEEC TWC WCRKSPACES, WKI ANO WK2 EOIH UF WHCSE OIMENIICAS ARE IA EY JN. FMT IS AUTCMATICALL

IF NEET TO CHANCE GRID ARRAY (THAT IS THE LIMENTICNS CF C ANC THE WOKK SPACE(S)) THEN MLST CALL RECCA (AC ARGUMENTSI THLS: 


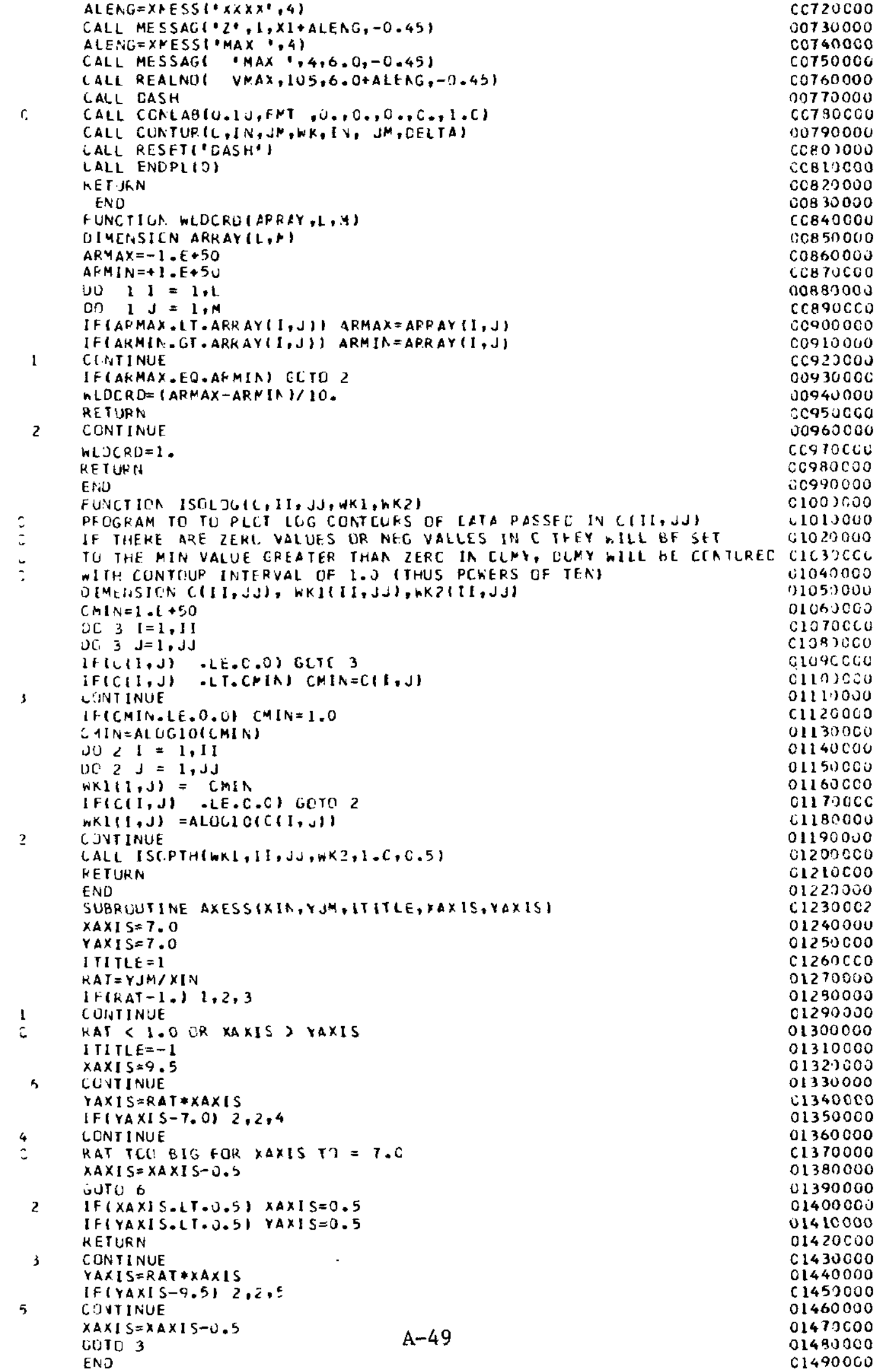

